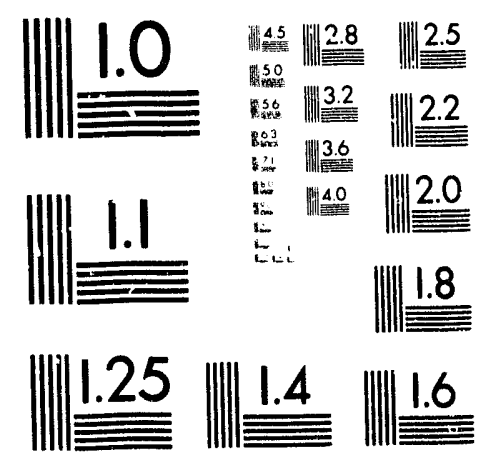



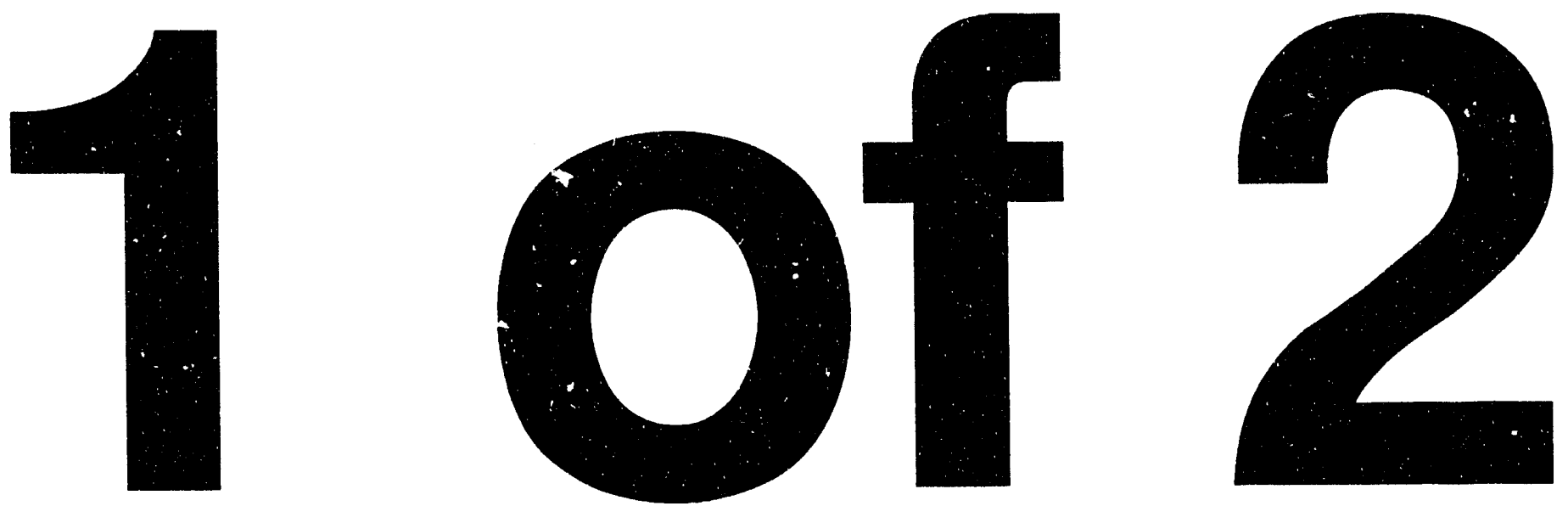


\title{
The Best of GYMNOS: A Users Guide
}

\author{
D. W. Hewett and D. J. Larson
}

August 1993

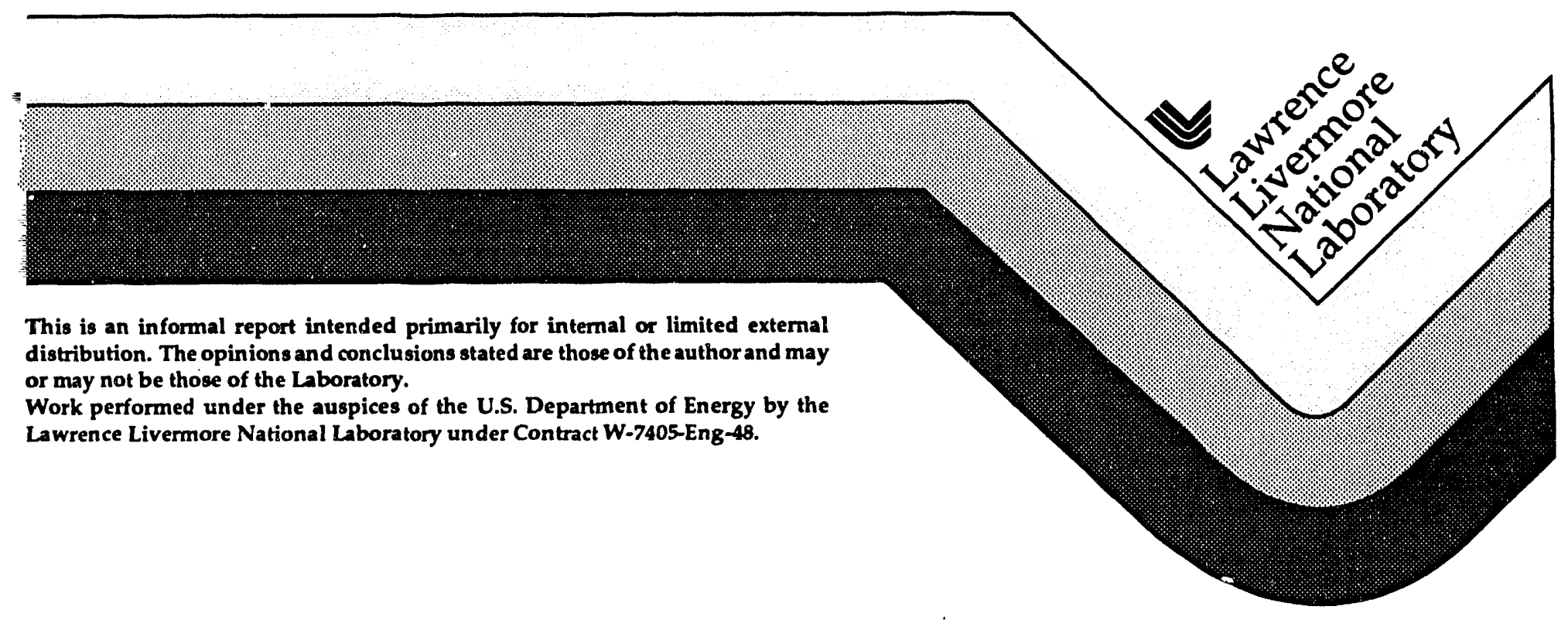




\section{DISCLAIMER}

This document was prepared as an account of work sponsored by an agency of the United States Government. Neither the United States Government nor the University of California nor any of their employees, makes any warranty, express or implied, or assumes any legal liability or responsibility for the accuracy, completeness, or usefulness of any information, apparatus, product, or process disclosed, or represents that its use would not infringe privately owned rights. Reference herein to any specific commercial products, process, or service by trade name, trademark, manufacturer, or otherwise, does not necessarily constitute or imply its endorsement, recommendation, or favoring by the United States Government or the University of California. The views and opinions of authors expressed herein do not necessarily state or reflect those of the United States Government or the University of California, and shall not be used for advertising or product endorsement purposes.

This report has been reproduced directly from the best available copy.

Available to DOE and DOE contractors from the Office of Scientific and Technical Information

P.O. Box 62, Oak Ridge, TN 37831

Prices available from (615) 576-8401, FTS 626.8401

A vailable to the public from the National Technical Information Service

US. Department of Commerce

5285 Port Royal Rd.,

Springfield, VA 22161 
The Best of GYMNOS: A Users Guide

\author{
D.W. Hewett and D.J. Larson \\ University of California \\ Lawrence Livermore National Laboratory \\ Livermore, California 94550
}

I. Introduction, History, dt Acknowledgement....................

II. Overall Philosophy and Verbal Flowchart..................

III. Initialization. $\ldots \ldots \ldots \ldots \ldots \ldots \ldots \ldots \ldots \ldots \ldots \ldots \ldots$

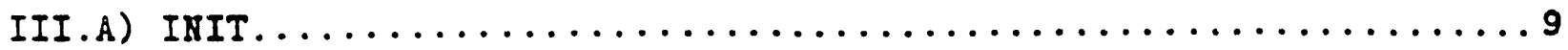

III.A.1) GEOSET called by INIT (See V. BCSET) ................

III.A.2) Species Input; Control Arrays NSTPROP, BEAM...........10

III.A.3) Macroscopic Profile Generation using INPROF............11

III.A.4) Particle initialization........................

III.A.4.a) Particle Parameter RPPSP Computed and

Buffering Initialized with SETB..............12

III.A.4.b) Particle Initialization

using Particles/Cell NPARTK.................12

IV. The Central Time Integration Loop....................... IV.A)) TRANSport of the particles from TIME to Time+DT...........17

IV.A.1) Start Ner Species, AUTOVLM, DPARTS, DPART

Call PBCSET (See V. BCSET), Preset E \& B.............18

IV.A.2) Call PARMOVe-The Particle Time Integration.............. 19

IV.A.2.a) Main Boris Push, Call TRAP,

First Order Advancz for Advanced Velocity Moments.....19

IV.A.2.b) TRAP of the Particles Outside Plasma Region........23

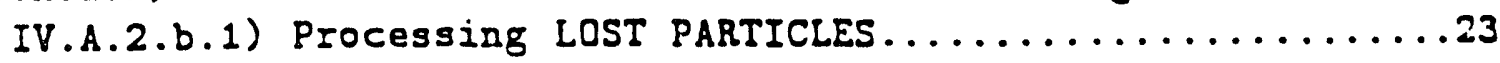

IV.A.2.b.1.a) Determining Closest Corner..............23

IV.A.2.b.1.b) Perfect Reflection or Delete..............24

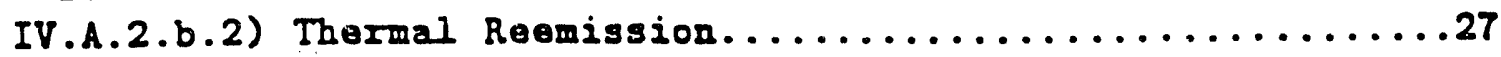

IV.A.2.b.2.a) Controls from PBCSET..................27

IV.A.2.b.2.b) Mechanics..........................28

IV.A.2.b.3) Injection and Field Emission................29

IV.A.2.b.3.a) Controls from PBCSET..................29

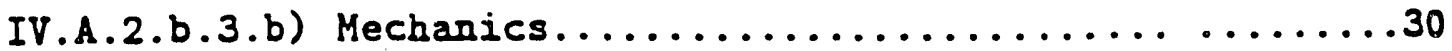

IV.A.3) Finish a Species, AUTOVLM, Restore E \& B, DAPARI

Convert Rav Counts to Densities, Save Bistories.........32

IV.B) Call DSOURCE (See VI. Diagnos) ........................ 32

IV.C) Call FIELD-The The Director of the Field algorithm,

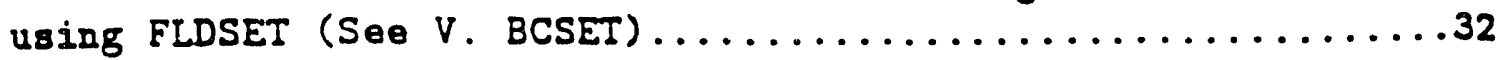

IV.D) Call DFIELD (See VI. DIAGNOS) ........................ 
V) User Specified Physics Routines

V.A) Macroscopic Initial Profile Generation by INPROF..............................................36

V.B) User Specified Boundary/Structure Physics in BCSET

V.B.1) GEOSET

B.B.2) PBCSET

V.B.3) FLDSET

VI) User Specified Diagnostics in DIAGNOS

VI.A) DPART Generates Particle Phase-Space Plots. .49

VI.B) DAPART Generates Particle Position-Space Plots................................................49

VI.C) DASPECS Plots Macroscopic Source Terms from Each Species...........................50

VI.D) DSOURSE Plots Species-Summed Macroscopic Source Terms............................50

VI.E) DFIELD Plots Potentials and Fields A, Phi, E. and B..........................................50

VI.F) HISTORY Plots Histories After "Unraveling" Pointer..........................................50

VII) Running GYMNOS

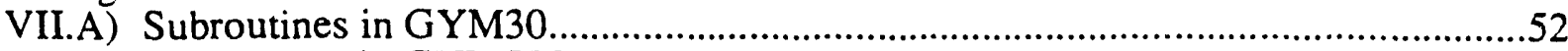

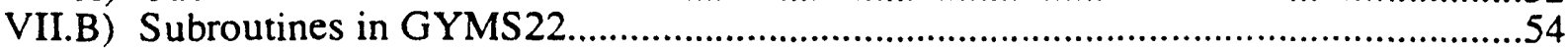

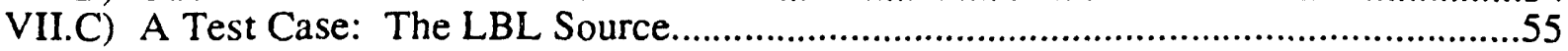

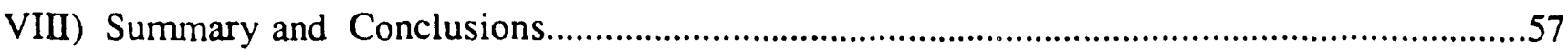

\section{APPENDICES}

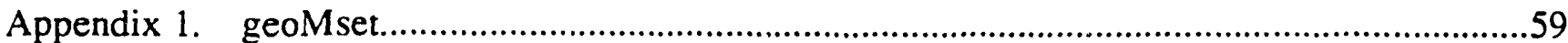

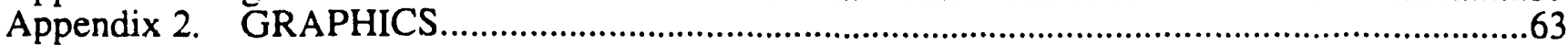

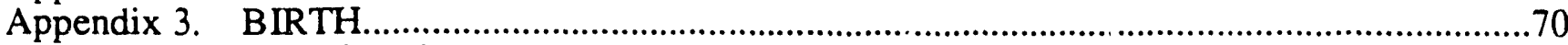

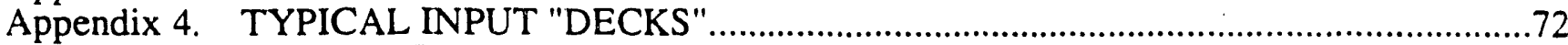

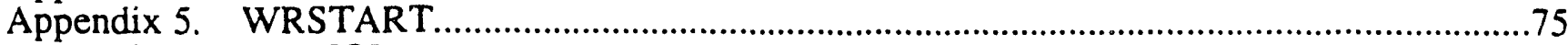

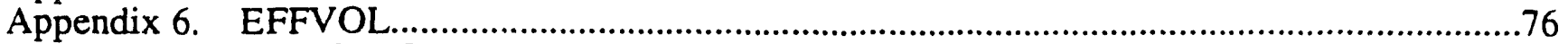

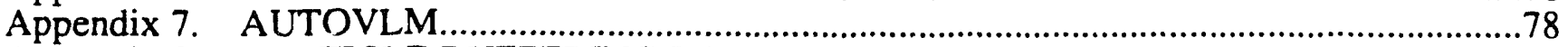

Appendix 8. PARTICLE BUFFERING ROUTINES: SETB, GETB, RESETB ……………….....79

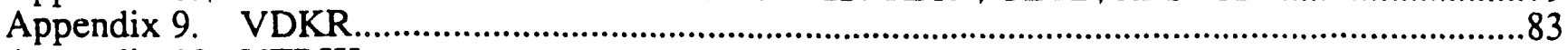

Appendix 10. VTRIK

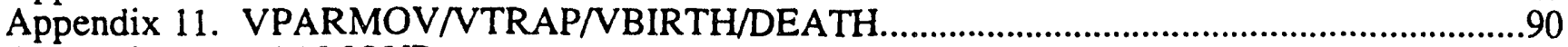

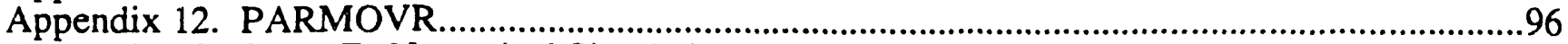

Appendix 13. Santa Fe Numerical Simulation Abstract..........................................................98

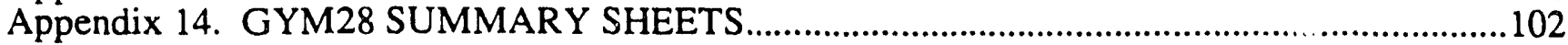




\section{Introduction}

The code GYMNOS is a 2.5D magnetostatic PIC code that is distinguished from the multitude of similar codes by its ability to handle boundary conditions and internal structures. This code was started (see next section) during the period starting from December 1987 and the major sections were completed and in routine use by the end of 1990 . The code is written in $\mathrm{R}-\mathrm{Z}$ geometry (axisymmetric $\mathrm{m}=0$ only) but could easily be modified to handle an X-Z configuration. The several competitors of this code, having similar (but we believe less general) capabilities, distinguish themselves in a different way: They have very formal, thick instruction manuals and usually command an army of people to maintain both the code and said manual. We are here attempting to convince the reader that GYMNOS is better in these two regards. Offered here is a description of a code with a great deal of physics capability coupled with no interactive graphics capability and very little system capability. These later two subjects contribute most of the complexity in both the code and manual in the competition! Expressed another way, the user will almost never be thwarted by unexpected system changes on Friday afternoon. The price extracted is that the user is required to monitor/babysit runs so that output is "hand-delivered" to the output medium and properly archived to prevent loss. A further spinoff of this simplicity is that "single pilot" operation is easy., And, the only manual is this modest document.

\section{History and Acknowledgements}

During the Christmas holidays in 1987, I found myself with an unusual opportunity. My employer, LLNL, found it necessay to allow its staff to take required racation between Christmas and New Year and, with my second child about to be born (1/12/88), I found the time to try some new ideas concerning techniques to impose boundary conditions orl internal structures in my favorite elliptic solving algorithms using ADI. As you will discover reading this, I was able to incorporate almost complete generality for each mesh point into an algorithm that could be completely vectorized using key or templete arrays.

The success of this venture left me with a very versatile algorthm that could quickly solve not only the Poisson equation but also the curl curl operator. After a few test cases around $r=0$ that suitably humbled me, I embarked on the construction of a PIC code that could serve as a vehicle for a renewed Darwin effort and a host of fluid electron, PIC kinetic ion models. After a series of lighthearted names, the name GYMNOS looks like it will stand the proverbial test of time for the basic code. The best justification is that this bare-bones vehicle for all the more sophisticated models to come is inamed the Greet word for naked.

As it turned out however, this "basic" code now has developed many capabilities. There is much to be done even with simple internal structures. GYMNOS now has the ability to generate its own internal structures given the essential information that it have a "bar" or "pie" cross section in the R-Z plane with a starting and ending point plus a width for the bar and a center, radius, and starting and stopping azimuth for the pie. Particles (perhaps multiple species) may be perfectly reflected, fully absorbed, absorbed with a fraction thermally reemitted, field-emitted using the self-consistent electric field, or injected with prescribed density, normal velocity, temperature, and temperature anisotropy: 
from any of these structures. The particle pusher itself is a Boris pusher that is fully vectorized.

The code accumulates particle and stores both particle and field data on cell corners. Internal structures are defined only on cell corners or lines that join nearest neighbor corners. The above mentioned particle absorption, injection, field emission, and reflection also take place at such corners (but thermal reemission comes from the "surfaces" between cell corners on structures surfaces). "Raw counts" from particle accumulation are converted to true densities in a manner that automatically accounts for the reduced volume of some of these cells adjacent to internal boundaries.

I experienced the joy of having a graduate student "come up to speed" during this period. David J. Larson (UC Davis) suffered through most of the development of the particle/structure interaction. His participation ranged ranged from "sounding board" in the early stages to bug finder/fixer as he applied GYMNOS to the MIRRORTRON concept. David is now quite capable of conceiving and buiiding enhancements in his own right: the relativistic particle pusher is wholly his implementation of the relativistic ideas in Birdsall and Langdon applied to our RZ Boris pusher. David is now implementing the "Streamlined Darwin Field" model using the new coupled equation solver he helped to invent. He is building this new capability into a variant of GYMNOS that he calls BEAGLE (the name of the ship that carried THE Charles Darwin on his origin-of-thespecies voyage!). I suspect we will all hear much more from this young physicist in the years ahead.

Dennis W. Hewett 10/21/91 


\section{Overall Philosophy and Verbal Flowchart}

What follows is a description of the software of GYMNOS. The description is necessarily given on several levels, starting with the most cursary overview.

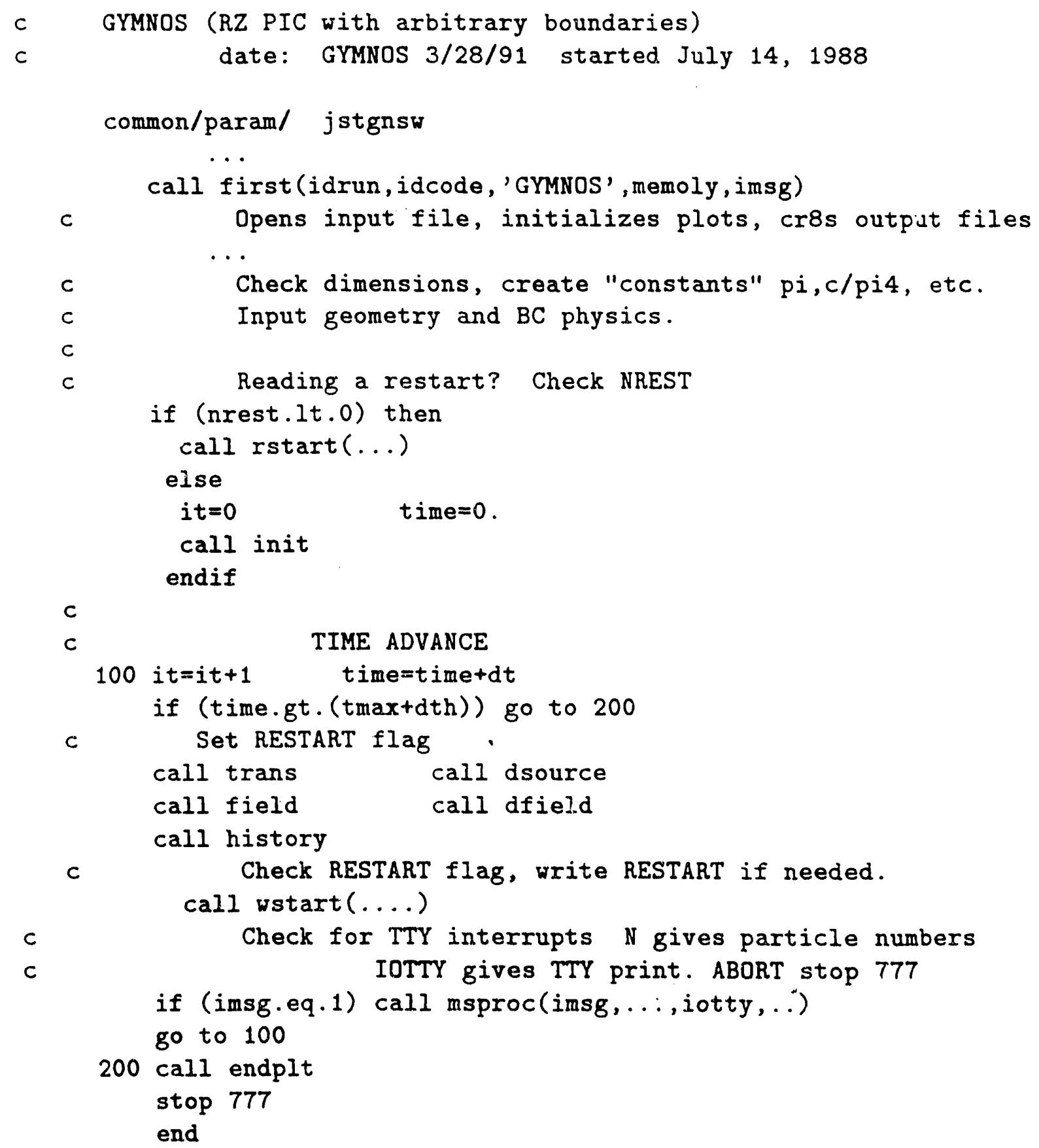

1) In the beginning, the code sets physical constants, creates files, initializes graphics, and opens the input file. This is done by a call to subroutine FIRST.

2) Namelist NDATA is read next. Most importantly, the namelist contains a time limit 
TMAX, time step size DT, plot intervals and a switch NREST that determines whether the code starts from an existing restart file or from a new initialization. If this is a new initialization the data in NDATA that defines the simulation region (including provision's for internal structures other than the default configuration) will be used. A parameter. JSTGNSW, tells the code either to use the default sturcture configuration, to expect to find more information in the input file, or to quiry the tty immediately after start up for additional sturcture information. Included here is also a switch controling the initial plasma configuration to be used-often signaled by ISTATE-and various voltages, time delays, and turnon and turnoff times.

3) The run is actually initialized by a call to routine INIT. INIT first causes the code geometry to be initialized via a call to GEOSET. GEOSET sets up at least 4 structures: typically, structure number NSTR $=1$ is a structure at. RMIN containing all the points $I=1$ for all $J$. Similarly, NSTR $=2$ contains all the points at $r=R M A X$, or $I=N R 1,1 \leq J \leq N Z$. NSTR $=3,4$ are the boundaries at ZMIN,ZMAX respectively. Structures 3,4 will not exist if these boundaries are periodic. Just which structure the corner points go into depends on the desired physics. STRGEN is the array which contains the controlling information and GEOSET, along with possible changes from input, sets this array. It then calls GEOMSET which set more control arrays such as SKEY, NSTRUCT, KORNT, KCIJ, KCORR. More details are available in the discussion of the entries in BCSET.

INIT reads in a namelist NECHSP for each species that includes a species name NAMESP, the initial number of particles NP, the maximum number NPMAX, the real charge per particle, the real mass per particle, the minimum and maximum density, the temperature (with possible anisotropy), and drift speeds.

Equally important, these species namelists bring information that set the physicá properties of the structure surfaces. Properites such as particle reflection. absorption and thermal reemission, field emission, and injection are carried in the arrays NSTPROP and BEAM. NSTPROP(NSTR,N) determines which species are active on a given structure. BEAM(NSTR,N,8) caries more essential injection/thermal reemission/field emission parameters such as density, normal velocity, anisotropy, and turnon/turnoff times.

For each species N, INIT calls subroutine INPROF for each grid point. INPROF return a cell center density, vector drift velocity, temperature, vector electric $E$ and magnetic $\mathrm{B}$ fields. Using the the curl of $\mathrm{B}$ as the source, INIT call a Poisson solver that returns the initial vector potential $A$.

Particle initialization is the next task. INIT calls SETB to initialized the particle storage and buffering required. Particles can now be created and stored in buffers that are acessed using calls to GETB. After determining several necessiry parameters, most importantly the parameter Real Particles Per Simulation Particle RPPSP for each species, the the NP initial particles for each $N$ are allocated to the various cells so that when we sum the particles and scatter them to the mesh, we recover roughly the same macroscopic moments we have defined in INPROF. This check is precisely what is accomplished in the bottom of INIT when a time step with DT $=0$ is performed.

4) The main time integration loop is in the MAIN routine, starting with statement label 100. First subroutine TRANS is called. This accomplishes a time step for all species and the accumulation of plasma source terms for each species as well as the net plasma 
charge and current densities. TRANS calls various entries in DIAGNOS as needed to get diagnostics during and after each species is integrated. MAIN calls the DIAGNOS entry DSOURCE to diagnos the net plasma sources and then calls FIELD which orchastrats the construction of new field components given the newly updated plasma sources. An entry DFIELD in DIAGNOS then diagnoses these new fields. A HISTORY entry in DIAGNOS is next called as needed as is a procedure NEEDF3 to close a plot file and open a new one. Finally, a restart file is written periodically and, almost always, when TIME exceeds TMAX.

5) MAIN rloses all files with a call ENLPLT for graphics and a STOP 777 , the usual signal for normal termination.

The code encompasses roughly 10,000 lines of FORTRAN, including the routines directly called by the five steps outlined above, a support binary package containing the Poisson solvers and related routines, and a plot library. There is a wealth of essential detail in these support routines. Many are essential parts of this "toolkit" that has been growing and evolving since the the beginning of computers. The biggest since source was probably Claire Nielson and his colleagues at LANL circa 1973-they probably won't recognize much other than some issues of style now and there is certainly no reason to hold anyone other than DWH responsible for accuracy and lingering errors. Countless incidents have convinced us that such errors will always exist and will materialize when new parameter regimes are attempted. However, we also believe that these bugs have little effect on the results of the test cases presented here.

A final comment, this code is written entirely in CGS units with temperatures in $\mathrm{eV}$ and most all mesh quantities are defined at cell corner. Generally formulas can be found in the NRL FORMULARY. We now discuss the various sections of the code in greater detail. 


\section{Initialization}

Having already seen the main time loop in the previous section, we now discuss bits and pieces of the code that require extra discussion to flesh out the picture that is always contained in the Fortran. The Fortran source is the ultimate reference. Right or wrong the code does only what it is told; the words that we add here only convey the concept of the algorithm that we intend for the code to accomplish.

DWH almost never uses explicit type declarations. Dennis makes fewer mistakes if he adheres to time honored integer and floating point variable names. DJL has been forced into this mode as well! Dennis has finally succumbed to the use of JR/Q/Z as the name of real current arrays.

real jr, jq, jz

I also use " $Q$ " or " $q$ " to represent $\theta$ throughout the code.

GYMNOS is built on a uniform retangular mesh with the I index associated with the $R$ or radial variable. $J$ is associated with the $\mathrm{Z}$ or axial variable. The mesh points run from $\mathrm{I}=1$ at $\mathrm{RMIN}$ to $\mathrm{I}=\mathrm{NR} 1$ at $\mathrm{RMAX}$ where $\mathrm{NR} 1=\mathrm{NR}+1$ and each mesh point is at a cell corner of the NR real cells in the problem. Similarly, J runs from 1 to NZ1 and spans NZ real cells in the axial direction. The code actually runs on a mesh that is dimensioned NR2 $\mathrm{X}$ NZ2 where $\mathrm{NR} 2=\mathrm{NR}+2$ and $\mathrm{NZ2}=\mathrm{NZ}+2$. All two dimensional quantities are defined on such meshes and dimensioned accordingly. The purpose is that I occasionally use a cell centered indexing scheme with a ghost cell on each end of the set of real cells already defined. A diagram of the r-axis makes things a little clearer.

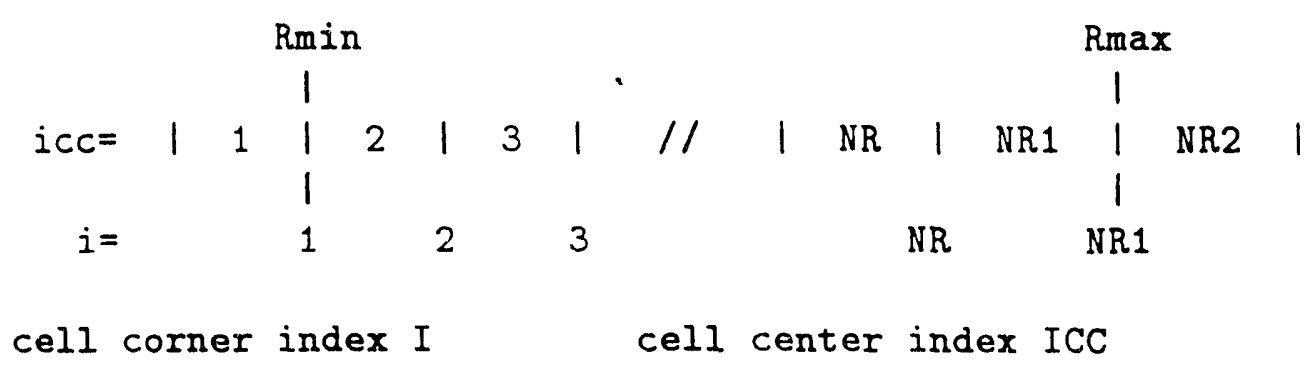

A parameter statement defines NR2 and NZ2 in the top of the main code (these are problem dependent).

parameter ( $\mathrm{nr} 2=73, \mathrm{nz2}=19, \operatorname{mxg} 1 \mathrm{~d}=73, \mathrm{nstmax}=8, \mathrm{ithmx}=2000, \mathrm{kcormx}=700)$

Soon after another parameter statement defines other useful grid parameters.

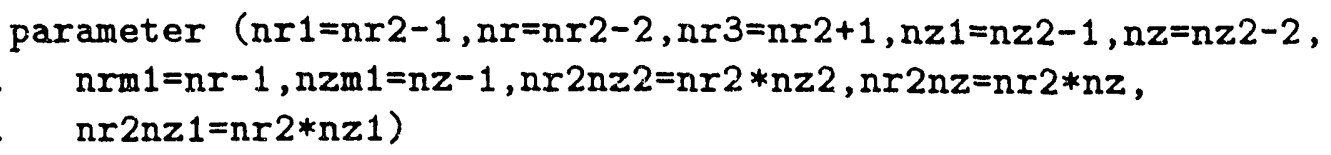


The physical dimensions that go with the counting indices can now be computed. If we are beginning a new run, INIT will be called here and these mesh parameters will be computed there. (If we are starting from a restart file, we need only compare a few things we have just input with what is already in the restart file to insure compatability.)

\section{III.A) INIT}

At the beginning of a new run, many things need to be set up and INIT is the subroutine that dose most of this-or at least directs it. To begin, we first compute those mesh lengths that go with the mesh discussed in the previous section. The dimensions of the mesh cells are straightforward and they and several related quantities are computed are computed and stored to ease the workload of constructing finite difference schemes throughout the code. (Caution! We put multiple Fortran statements on the same line to save paper-read left to right then down.)

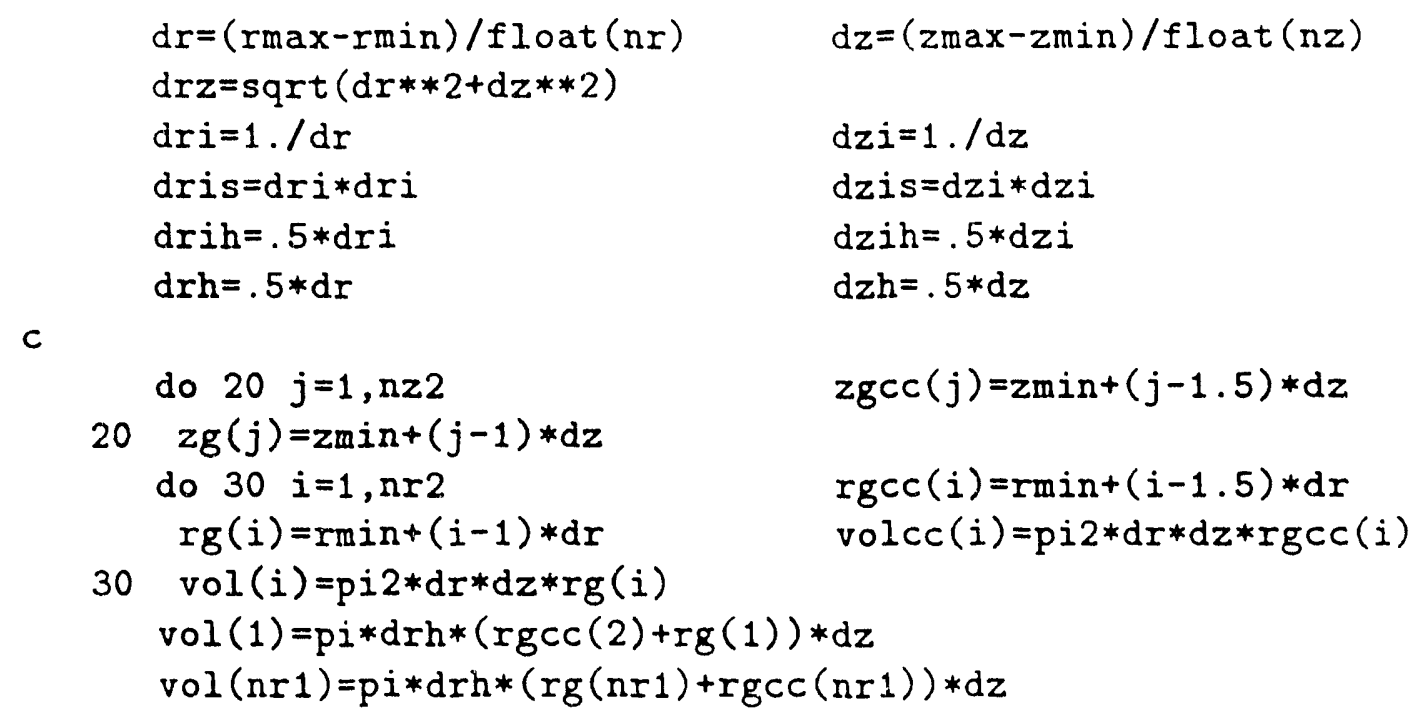

Note that RG, Radial Grid, is a cell corner quantity. RGCC is a Cell Center quantity. Similarly, the suffix $C C$ has the same meaning for the $z$-axis and for the cell volume definitions. Notice that VOL needs to be adjusted at each end to account for the "volume" outside of the simulation region.

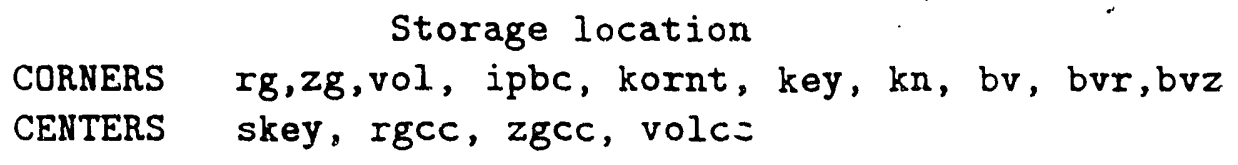

\section{III.A.1) GEOSET called by INIT (See V. BCSET)}

INIT has just set most of the elementary grid parameters. It now calls GEOSET to initialize the arrays that determine structures. To accomplish this GEOSET (a user/problem specific routine) will fill arrays NSTRUCT, NSTPROP, and STRGEN. Next GEOSET calls the routine GEOMSET that interprets STRGEN to build controlling arrays NSTRUCT, 
KCIJ, and KCORR that will be used almost everywhere else in GYMNOS. Details can be found in Section V. and Appendix 1.

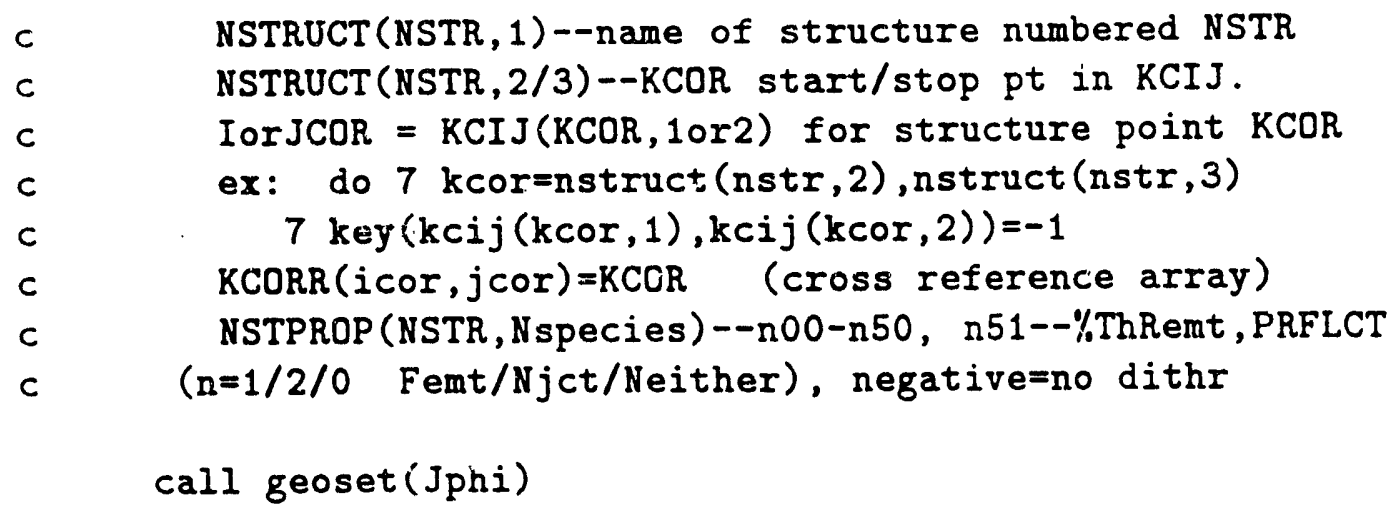

\section{III.A.2) Species Input; Control Arrays NSTPROP, BEAM}

In this section of INIT, we load the particle parameters species by species. In adition. species dependent beam and structure parameters are also input.

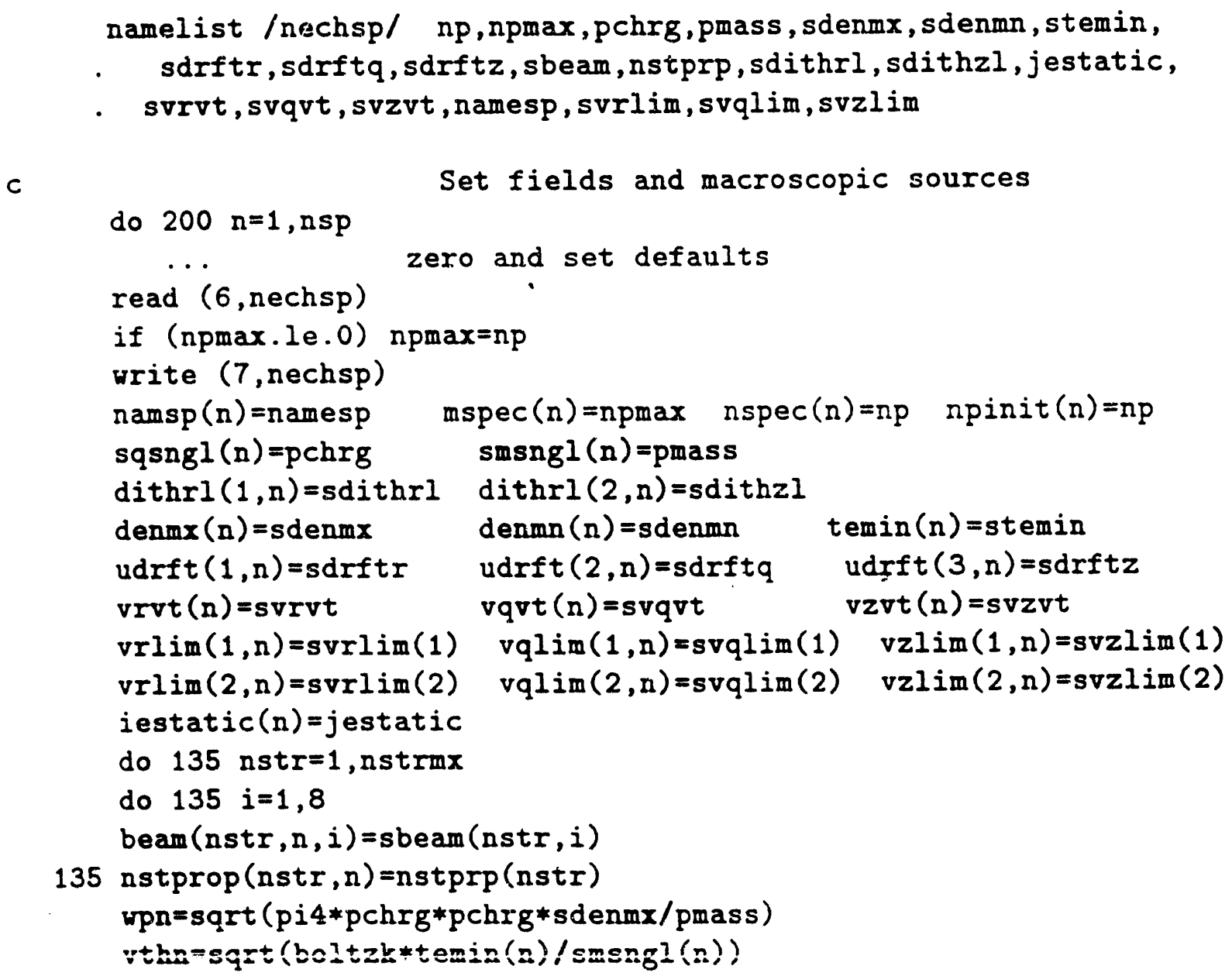




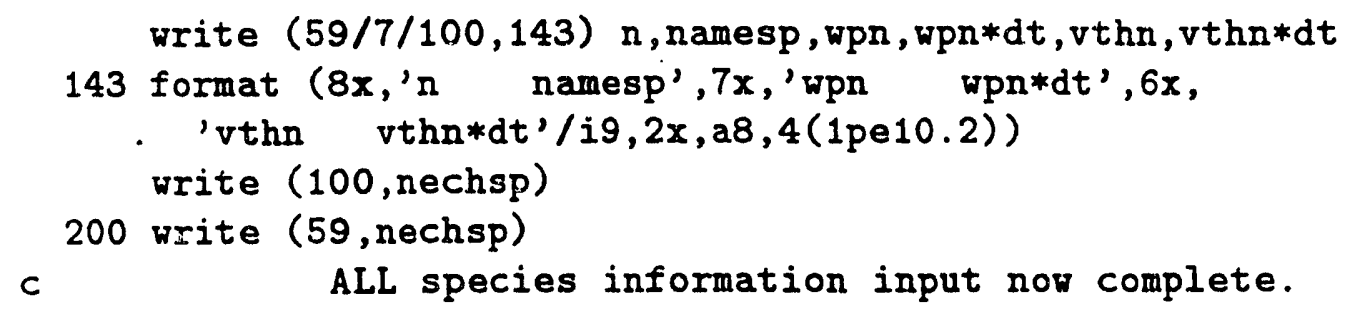

\section{III.A.3) Macroscopic Profle Generation using INPROF}

INIT calls INPROF for every cell center to get the ideal macroscopic moments for the PIC represented species. These cell centered moments serve as weight functions for the soon-to-initialized particle distributions. DWH first learned about this method from A.G. Sgro who had implemented these ideas in 1D. Initial field configurations could also be initialized this way but for elliptic field models return self consistent fields without need of any previous information given the instantaneous plasma source configuration. Other models DWH has written (such as zero- and finite-electron-mass hybrid models) used this procedure.

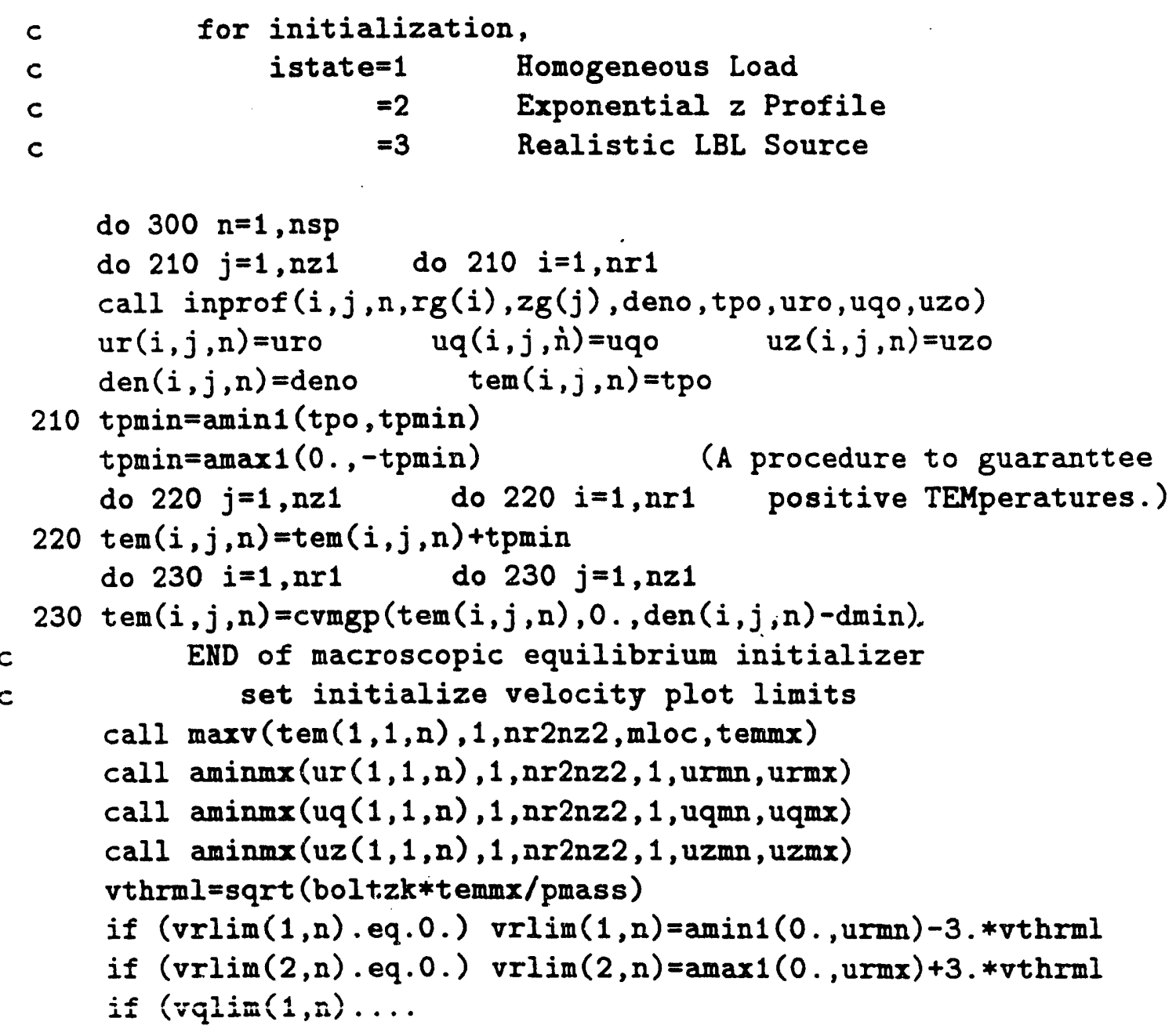


if $(\operatorname{vzlim}(2, \mathrm{n}) \ldots$

call aplotx (den $(1,1, n), \mathrm{nr} 2, \mathrm{ravg}$, zavg, rsc,zsc,

$-2,-2,-3,-2, n r 1, n z 1,1, n r 1, \operatorname{namsp}(n), 8$, $^{\prime}$ aplt den' $\left., 8,1,1,1\right)$

call cplot $(-2,-3, \mathrm{nr} 1, \mathrm{nz} 1, \mathrm{rsc}, \mathrm{zsc},-1,-1, \mathrm{nr} 2, \operatorname{den}(1,1, n)$,

$x$

-mcont , zc, 'Den', $3,{ }^{\prime} r$ ', 1, ' $^{\prime}$ ' $\left.^{\prime}, 1\right)$

300 call vplot $(-3,-3, n r 1, n z 1, r s c, z s c,-n c r,-n c z, n r 2$, $\left.\operatorname{ur}(1,1, n), \operatorname{uz}(1,1, n),{ }^{\prime} U r, z^{\prime},-4,{ }^{\prime} r^{\prime}, 1, z^{\prime}, 1\right)$

\section{III.A.4) Particle initialization}

III.A.4.a) Particle Parameter RPPSP Computed and Buffering Initialized with SETB

$$
\text { - . }
$$

$\mathrm{N}$ is species number TOTNRP...sum real prtcls each $\mathbb{N}$ $\operatorname{NPINIT}(n)=N P$...number of simulation particles requested NSPEC(n) ...instantaneous \# of species $N$ sparticles RPPSP $(n)$...real particles per sparticle (=TOTNRP/NPINIT(N))

...

Now Initialize Particles for each species mpb=mpbuf

call setb (nsp, mpb, mspec, nspec, nbspec, memoly, dimp, nheadb)

Call to SETB sets up $i / 0$ buffering of the particles where...MSPEC

contains the MAX \# of each.prtcl type. (See Appndx 8 on buffering)

header (PBUF, IPBUF) layout

PBUFH(1) = buffer number for this buffer

2 = \#artcls in this buffer (called NPTHISB)

3 = ultimate \# prtcl blocks/species--also in NBSPEC( $n$ )

4 = species number $=n$

$5=$ species name $=\operatorname{namsp}(n)=$ namesp

6 = time step number $=$ it

$8=$ species charge $=s q(n) \quad(\operatorname{pchrg}=s q(n) / \operatorname{mppsp}(n)=\operatorname{sqsngl}(n))$

$9=\operatorname{species}$ mass $=\operatorname{sm}(n) \quad($ pmass $=\operatorname{sm}(n) / \operatorname{rppsp}(n)=\operatorname{smsngl}(n))$

\section{III.A.4.b) Particle Initialization using Particles/Cell NPARTK}

dimension npartk(nr2,nz2)

equivalence (npartk $(1,1), \operatorname{rho}(1,1))$ 


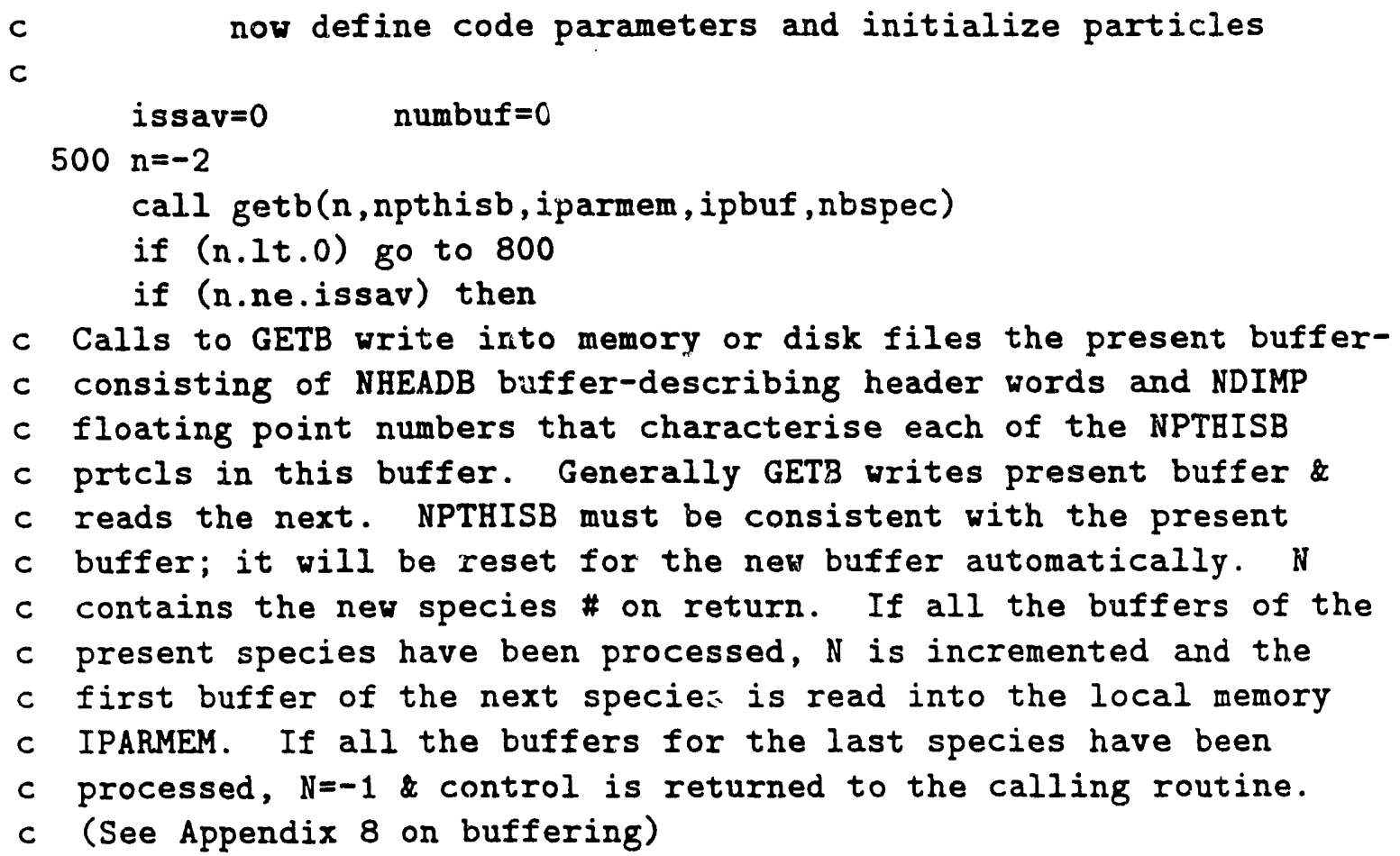

The section immediately below integrates the cell center DEN at the start of each species $\mathrm{N}$ to find the number of real particles that the entire simulation contains. (In DEN is zero everywhere but particle emission is expected, a uniform fill of the simulation region is aswsumed.) Next the array NPARTK is filled with integers that represent the number of simulation particles that must be created in each cell in order for that cell to have the expected real density. It is natural here to define for each species the quantity RPPSP $(N)$ (representing Real Paticles Per Simulation Particle). Now the quantities $\mathrm{SQ}(\mathrm{N})$ and $\mathrm{SM}(\mathrm{N})$, representing the real charge and mass per simulation particle can be determined.

c Start new species (remember DEN is cell centered) bamp $=0$.

do 545 nstr $=1$, nstrmx

545

bamp=bamp+beam (nstr, $n, 1)$

totnrp $=0$. refnrp $=0$.

do $550 i=2, n r 1$ do $550 j=2, n z 1$

$\operatorname{skyf}=1 .+\operatorname{skey}(i, j) * .5 *(1 .-\operatorname{skey}(i, j))$

totnrp $=\operatorname{totnrp}+\operatorname{den}(i, j, n) * \operatorname{volcc}(i) * \operatorname{skyf}$

550

refnrp=refnrp+bamp*volcc(i)*skyf

refnrp=refnrp+totnrp totnrp=amax 1 (totnrp,1.)

DEFISH $=0$.

ntot $=0$

do $560 j=2, n z 1$ do $560 i=2, n r 1$

$\operatorname{skyf}=1 .+\operatorname{skey}(i, j) * .5 *(1 .-\operatorname{skey}(i, j))$ 


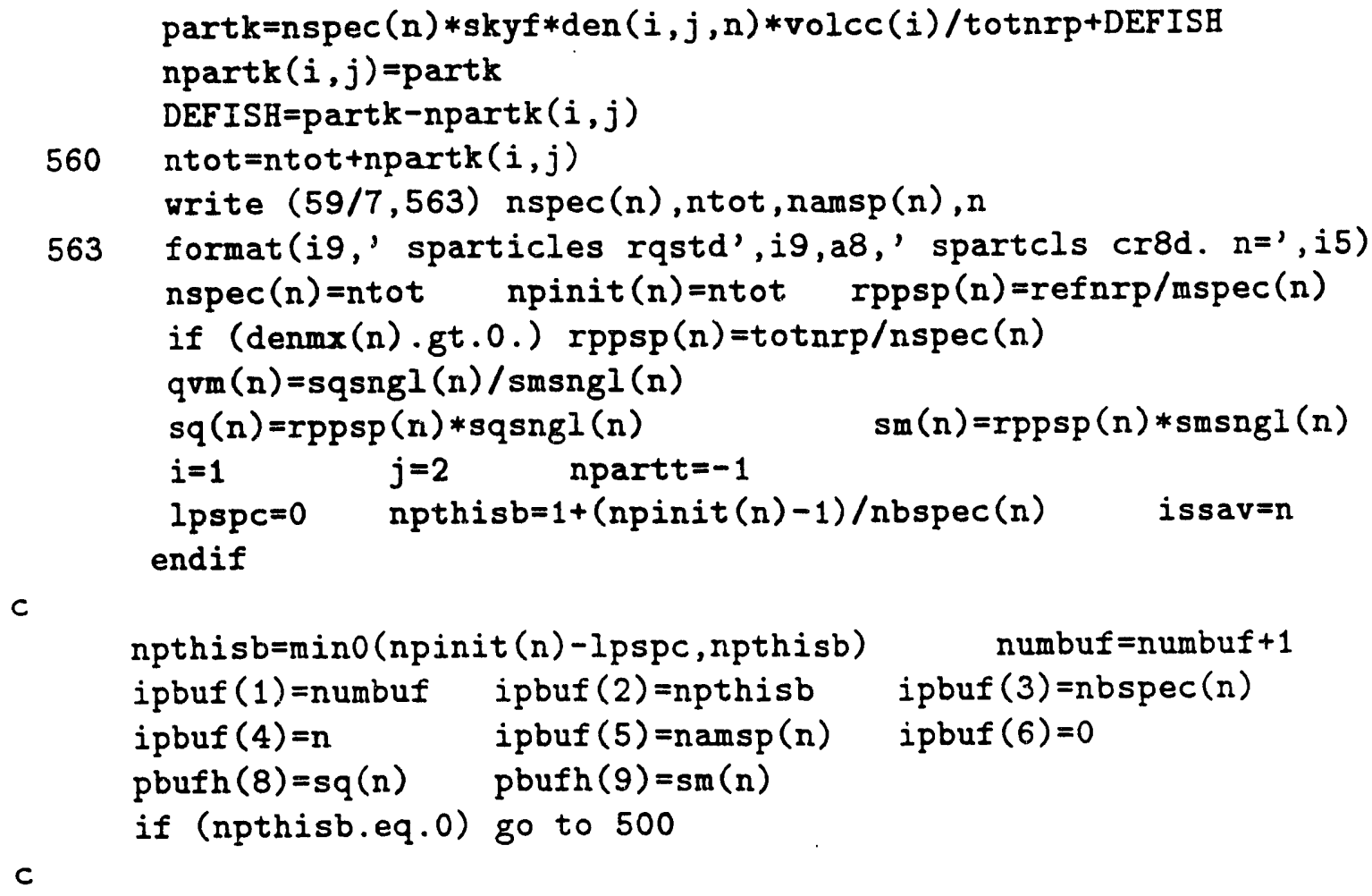

The next section describes the random creation within each cell the number of particles NPARTK $(I, J)$ needed to represent the desired density $D E N(I, J, N)$. As they are created, they are given random thermal velocities consistent with the temperature TEM(I,J,N), and anisotropy VR/Q/ZVT(N), and have a drift velocity $U R / Q / Z(I, J, N)$ added.

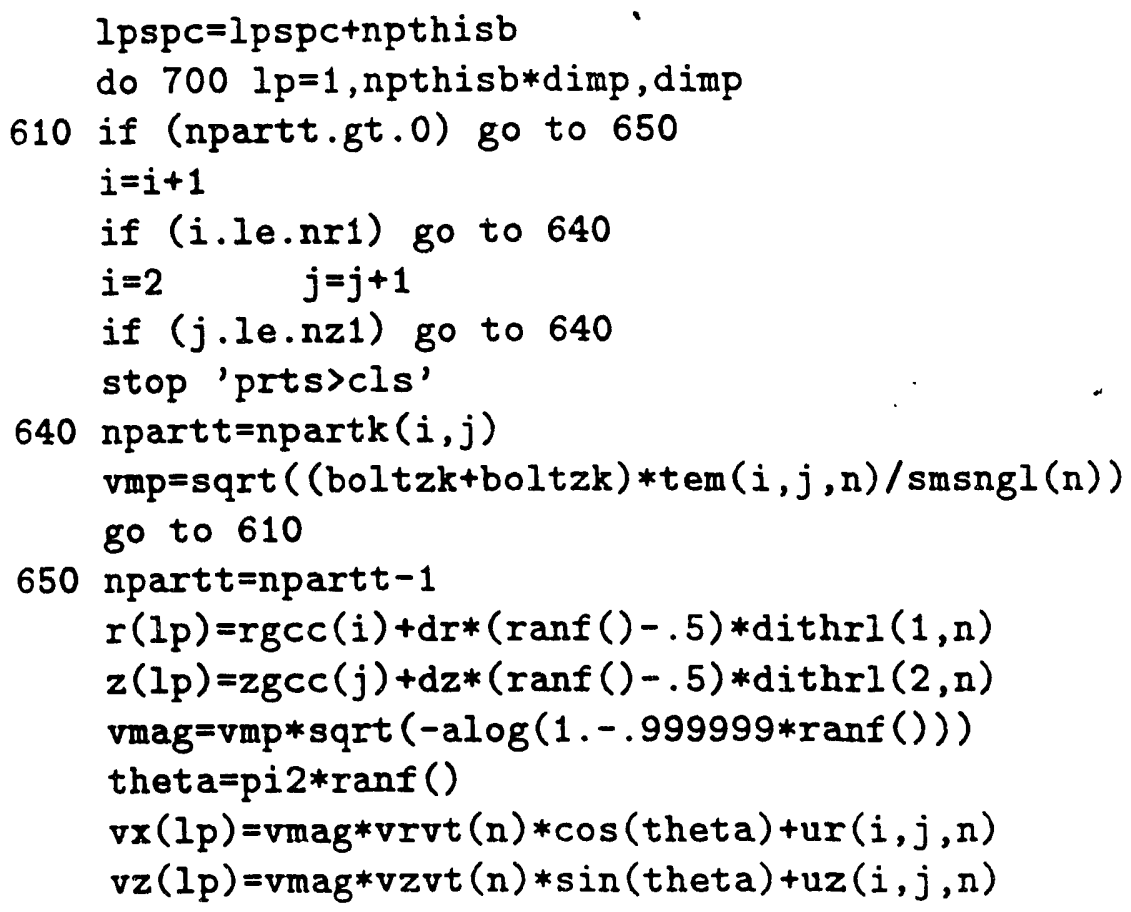




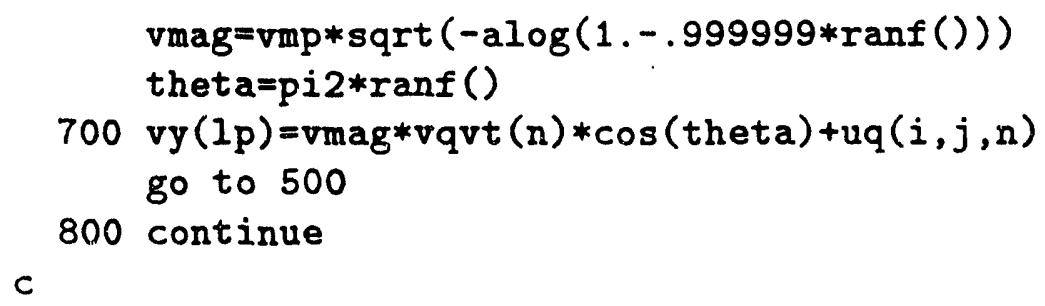

Finally, INIT sets the time step DT to zero and runs a complete time step just to fully initialize all the fields. A further benefit is that all the diagnostics run a TIME $=0$ so you really know and can prove the initial state of the simulation. (Important point, read between the lines here!)

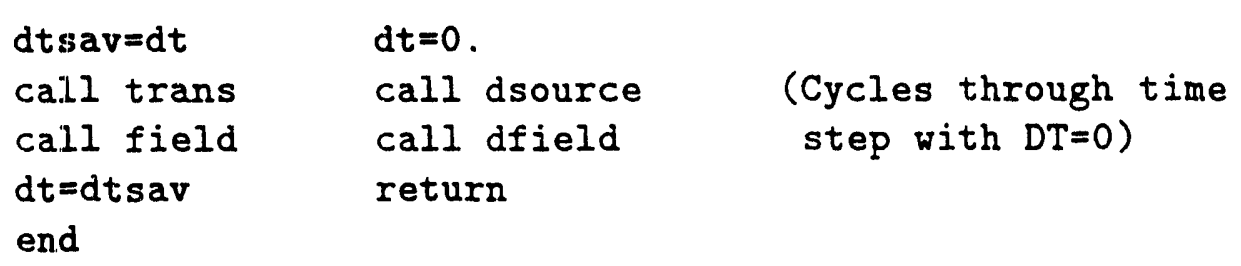




\section{The Central Time Integration Loop}

The main program MAIN directs the action of the entire code. MAIN contains not only the central the loop with periodic calls to diagnostics but also the calls to the initialization/RESTART routines as well as the procedures to start and clean up at the end of the run. These things will be discussed in due course; for now look at the central time integration loop-found about halfway through the MAIN routine.

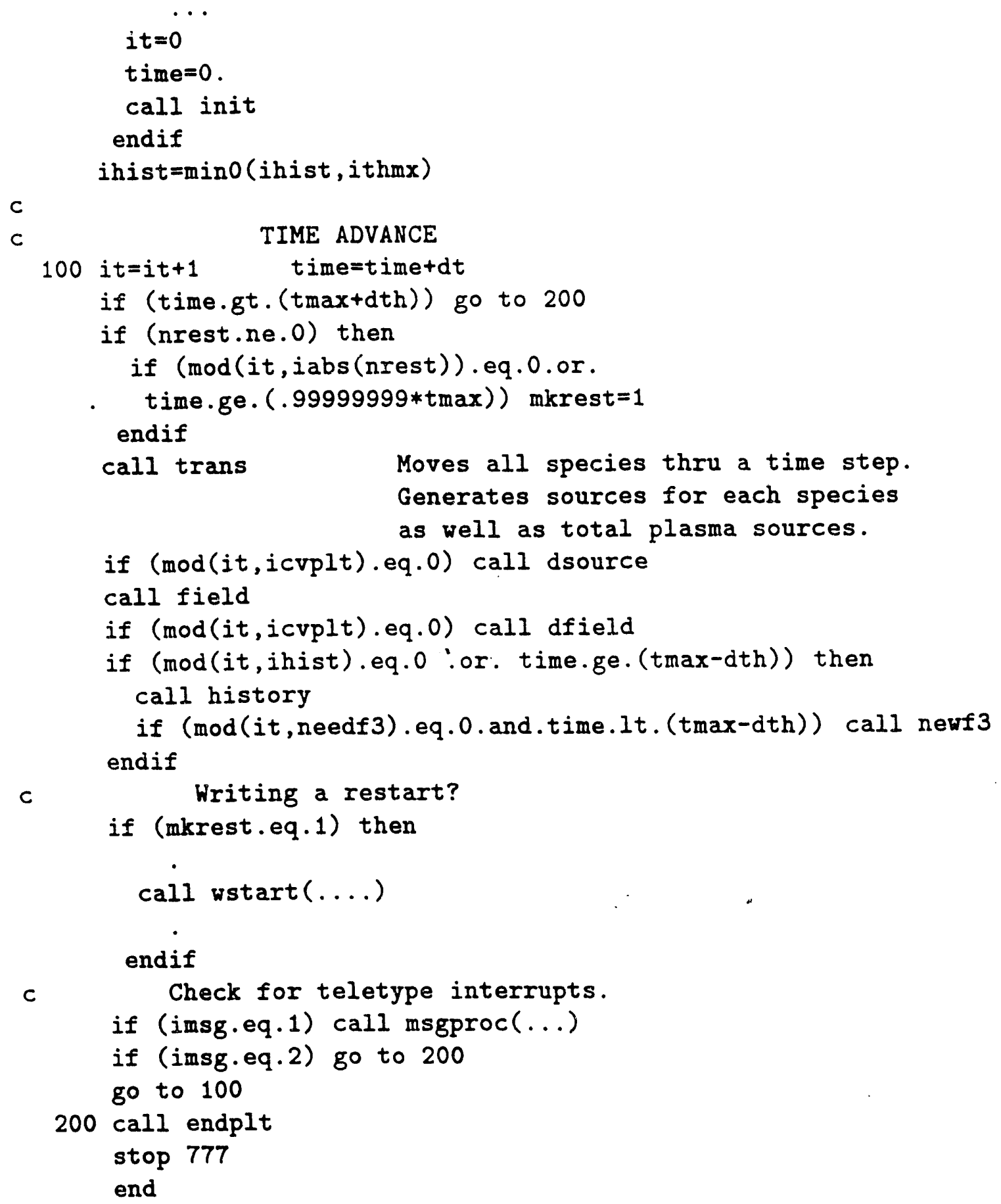


First note the loop on IT and TIME between 100 and 200. (I use all capitals to distinguish FORTRAN variables within the text). This is the time integration loop: IT is the time step count and TIME is the time in units of seconds. Control stays within these lines until TIME exceeds TMAX. The first call is to subroutine TRANS which integrates or "TRANSports" all plasma quantities (particles positions and velocities, plasma source terms) forward in time through one time step. The details of this process are discussed in later section B). For now it is only important to remember that TRANS is called with particle positions and velocities and $E$ and $B$ fields at time level TIME $E_{n}$ and it returns with the positions and velocities of the ions advanced by DT to $\mathrm{TIME}_{n+1}$. Also returned are the source terms for the number density DEN, mean flow velocity in the three directions UR, UQ, UZ, and the temperature TEM. [Throughout this write up and GYMNOS, Q or $\mathrm{q}$ is used to denote theta.] TRANS deals with any and all particle buffering and associated gymnastics to accomplish this time advance. TRANS also calls the diagnostics for each particle buffer as well as diagnostics after each species is processed. Next is a call to DSOURCE that plots and otherwise diagnoses source totals over species.

FIELD computes the potentials $A$ and $\Phi$ given the instantaneous particle source terms. A call is made to DFIELD which provides diagnostics, mainly graphical. for the new information available after the field integration. Likewise, a call to HISTORY, if it is time. call to plot selected time histories that may be stored by TRANS as the time integration progresses. We also check to see if it is time to close a plot file and open a new one by comparing the NEEDF3 parameter with the time step. Finally we check if we need to write a restart file at this time step and we check very time step for information from the terminal. When TIME TMAX, we flush the plot buffers with ENDPLOT and terminate with STOP 777, our normal termination indicater.

\section{IV.A) TRANSport of the particles from TIME to Time+DT}

The purpose of TRANS is to coordinate the cycling of particles through a particle pusher. The general strategy is to bring in a buffer of particle data and. if it is a new species type, "finish" the previous species and "start" the new species. Slight adjustments are made not to finish a species if we're just starting a time step nor to start a species if the buffer we have just finished pushing is the last of the species to be processed this time step.

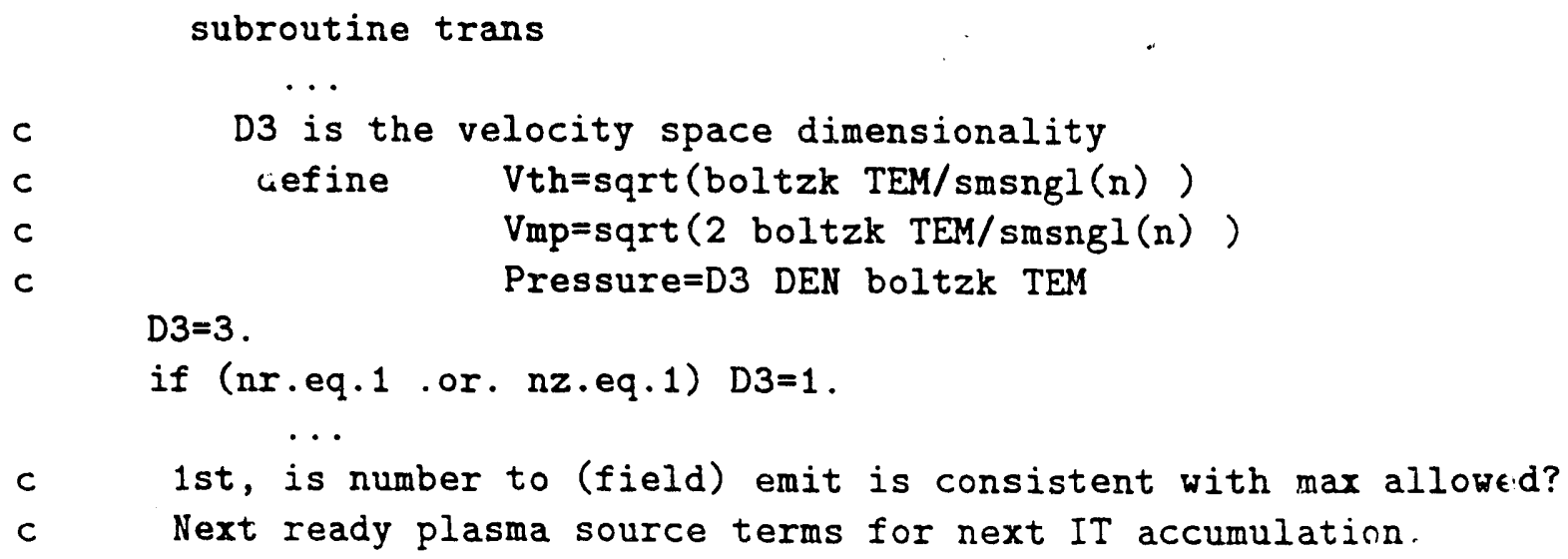


call getb(n,npthisb,iparmem,ipbuf,nbspec) \{Get 1st prtcl buffer\} go to 400 \{Skip to start species\}

c Top of particle species "loop"

100 call getb (js, npthisb,iparmem, ipbuf,nbspec) if (js.1t.0) go to 200 \{Last buffer processed, finish species.\} if (js.eq.n) go to 500 \{Species unchanged, go push buffer.\}

c

IV.A.1) Start New Species, AUTOVLM, DPARTS, Call PBCSET (See V. BCSET), Preset $\mathrm{E}$ and $\mathrm{B}$

C

$400 \cdot$ npthisb $=$ ipbuf (2)

c

$$
\begin{aligned}
\text { if }(\bmod (i t, i p p l t) . e q .0) \text { call dparts } \\
\text { \{Diagnostics PARTicle Start\} }
\end{aligned}
$$

AUTOVLM here (see appendix) Prepare VLIM's to find velocity extremes for prtcl plots NEXT time step.

call pbcset(Jphi) \{Set particle BCs for this species $N$ \}

c Zero accumlating arrays, $f i x E, B$ with species dependence hqvm $=.5 * q v m(n)$

hqumc $=$ hqum/C

$e r / q / z(i, j)=e r / q / z(i, j) * h q v m$

$b r / q / z(i, j)=b r / q / z(i, j) * h q v m c$

$500 \mathrm{n}=\mathrm{ipbuf}(4)$

c

now push the blk of particles

call parmov

AUTOVLM (see apdx 7) Find max/min velocities IF Pploting next IT

Call Diagnostic PARTicle DPART if Pploting NOW

if (mod(it,ipplt).eq.0) call dpart

go to 100

700 continue

C DAPART provides a diagnostics opportunity on particle quantities.

c Here we use the extra pass to get $R-Z$ space plots of particles.

if (mod(it,ipplt).eq.0) call dapart \{Diagnostics After PARTicles\}

return

end 
The point is simply that, with this storage arrangement, all quantities associated with the lpth particle may be accessed with the subscript LP. Don't be concerned about the use of $\mathrm{Vx}$ for $\mathrm{Vr}$ or $\mathrm{Vy}$ for $\mathrm{Vq}$-the explanation is found and discussed in the particle pusher section PARMOV.

NPTHISB and N (species number) are generated in GETB; They are carried in the header words IPBUF(2) and (4), respectively. They are appropriately set by GETB which expects the particle buffers, will contain only one type of particles (though adjacent similar particles may be plotted on the same plots), will be contiguous for each species $\mathrm{N}$. The change in species type is signaled by a change in JS that GETB gets by reading the fourth header word IPBUF(4). The end of the particle buffers of the last species is denoted by JS $<0$.

The call to DPART is used to produce phase-space plots as each buffer load of particles is processed.

\section{IV.A.2) Call PARMOVe-The Particle Time Integration}

IV.A.2.a) Main Boris Push, Call TRAP, First Order Advance for Advanced Velocity Moments

The particle time advance is accomplished by a Boris Pusher. It is reversible and second order in time. A derivation follows the Fortran.

The essential concepts are that the particles are all pushed as if they were on a Cartesian $X-Y$ mesh in the $R$-Theta plane. In axisymmetry all particles are considered to be rings of volume $R^{*} D R^{*} D Z$. At the beginning of each time step, a particle's position is considered to be at $X=R$ and $Y=0$ with $V x=V r$ and $V y=V q$. $V x$ and $V y$ at time $t_{n+1 / 2}$ are computed using $E x=E r$ and $E y=E q$. [Remember $Q$ or $q$ is used to denote $\theta$.] The particle is advanced to a new $\mathrm{X}$ and $\mathrm{Y}$ with these velocities. This is accomplished by the FORTRAN

subroutine parmov

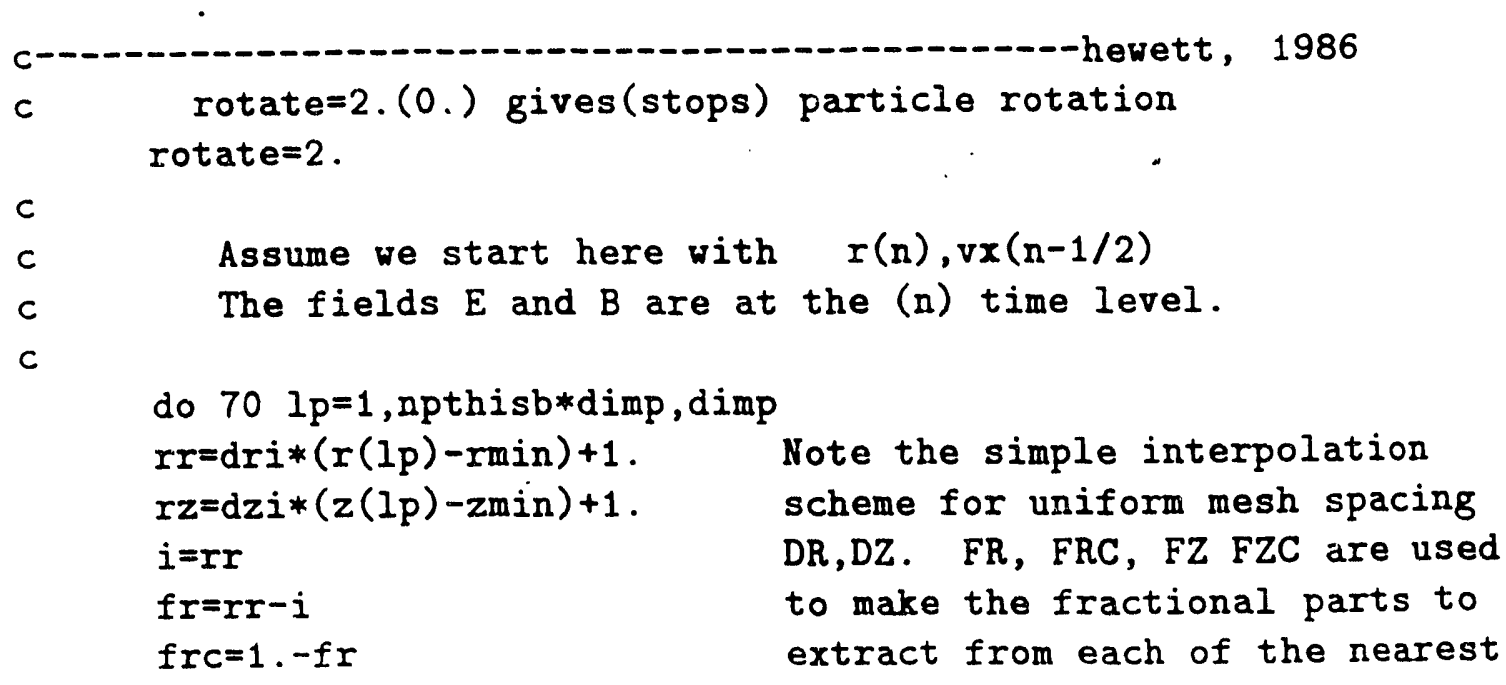


$j=r z$

$f z=r z-j$

$w 1=f r c * f z c$

$w 2=f z * f r c$

$w 3=f r * f z c$

$w 4=f r * f z$
4 cells to the particle location.

These are the interpolation

factors determining the contribution from each of the cells to the field at the particle location.
C

do half of $E$ push

eacclr/q/z=dt*(w1*er/q/z(i,,$j)+w 2 * e r / q / z(i, j+1)$

$+w 3 * e r / q / z(i+1, j)+w 4 * e r / q / z(i+1, j+1))$

$v x / y / z(l p)=v x / y / z(l p)+e a c c l r / q / z$ rotation follows

omgr/q/z=dt*(w1*br/q/z(i,j)+w2*br/q/z(i,j+1)

$+w 3 * b r / q / z(i+1, j)+w 4 * b r / q / z(i+1, j+1))$

$v x / y / z t=v x / y / z(l p)+v y / z / x(l p) * o m g z / r / q-v z / x / y(l p) * o m g q / z / r$

fact=rotate $/(1$. +omgr*omgr+omgq*omgq+omgz*omgz)

$v x / y / z(I p)=v x / y / z(I p)+f a c t *(v y / z / x t * o m g z / r / q-v z / x / y t * o m g q / z / r)$ rotation finished

do remaining half of $E$ push

$v \times h=v x(l p)+e a c c l r \quad v y h=v y(l p)+e a c c l q \quad v z(l p)=v z(l p)+e a c c l z$

now advance to $r(n+1)$ in order to find $v x(n+1 / 2) \quad 4 / 3 / 90$

$x=r(1 p)+v x h * d t$

$z(I p)=z(I p)+v z(I p) * d t$

dyyy $=$ vyh $* d t$

$r(1 \mathrm{p})=\operatorname{sqrt}(\mathrm{x} * \mathrm{x}+\mathrm{dyyy} *$ dyyy $)$

costh $=x / r(l p)$

$v x(1 p)=\operatorname{costh} * v x h+\operatorname{sinth} * v y h \quad v y(1 p)=-\operatorname{sinth} * v x h+\operatorname{costh} * v y h$

70 continue

call trap

push completed, now TRAP LOST particles

After TRAP extrapolate $v x(n+1 / 2)$ to $v x(n+1)$, using new positions $r(n+1)$ and fields $E(n+1 / 2)$ and $B(n+1 / 2)$, to accumulate "raw count" sources at the desired time level.

do $2701 p=1$, npthisb*dimp, dimp

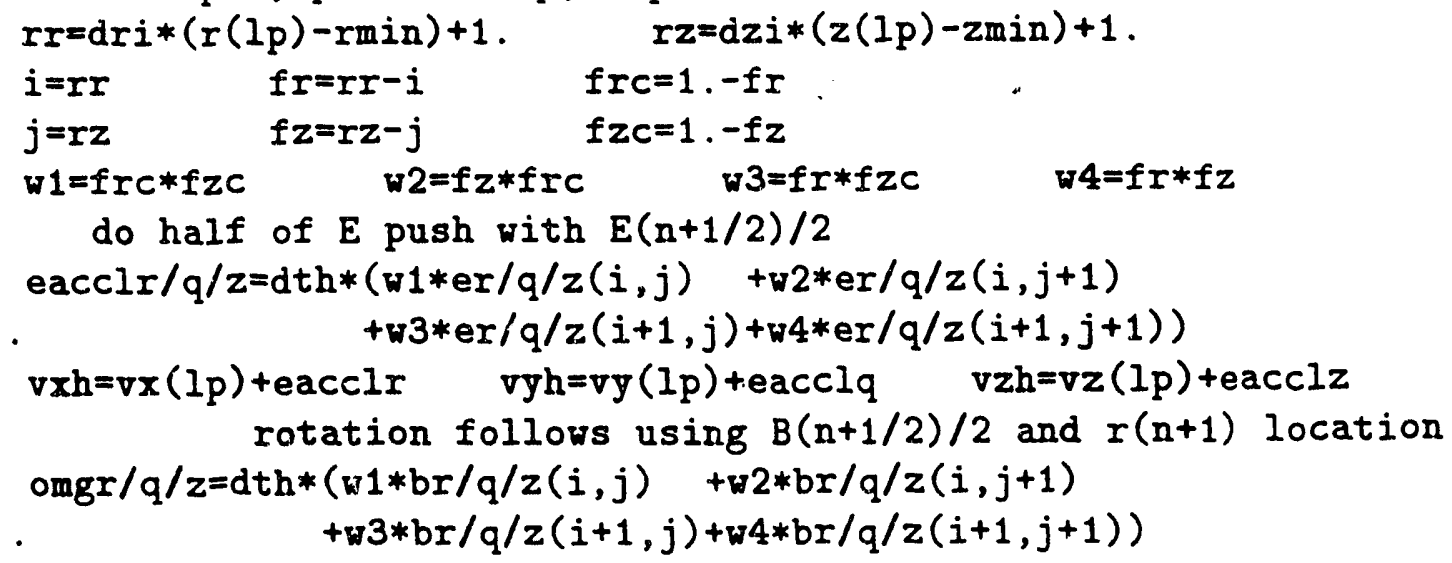




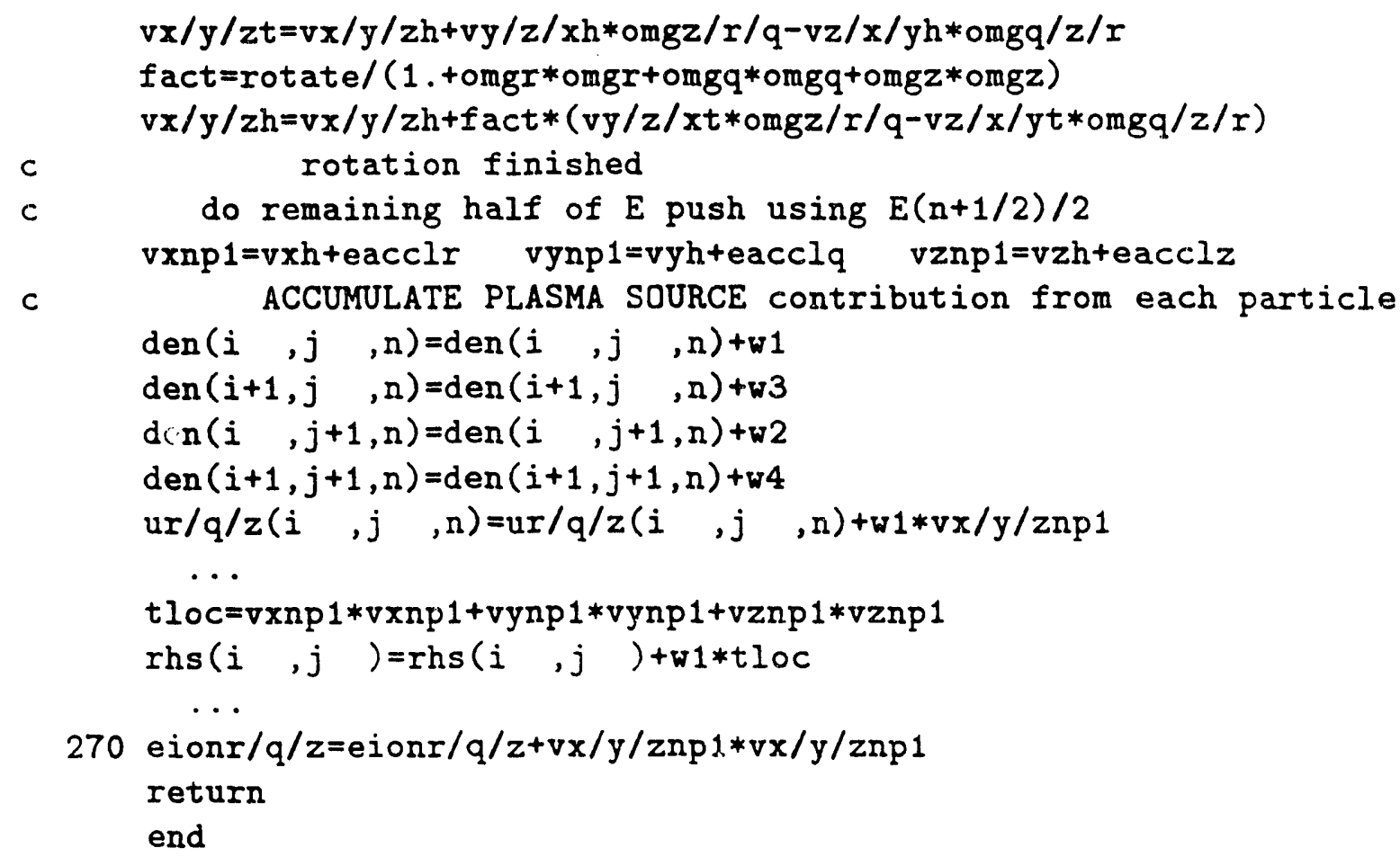

The formulas can be obtained by first writing the desired scheme

$$
V_{n+.5}=V_{n-.5}+\Delta t\left(E_{n}+\frac{V_{n+.5}+V_{n-.5}}{2} \times B_{n}\right)
$$

where the charge, mass, and $\mathrm{C}$ have been incooporated into the fields as appropriate. We may choose to decompose this expression into the following three parts:

$$
\begin{gathered}
V_{1} \doteq V_{n-.5}+\frac{\Delta t}{2} E_{n} \\
V_{2}=V_{1}+\left(V_{2}+V_{1}\right) \times \Omega \\
V_{n+.5}=V_{2}+\frac{\Delta t}{2} E_{n}
\end{gathered}
$$

where $\Omega=\Delta t B_{n} / 2$. The clever idea in the Boris pusher is that the middle expression that describes a time-centered implicit rotation can be solved without resorting to a $3 X 3$ linear system for $V_{2}$. To see this, rewrite the middle equation as

$$
V_{2}-V_{2} \times \Omega=V_{1}+V_{1} \times \Omega
$$

and let both sides of the equation equal yet another variable $V^{\prime}$. We next add each of these equations together, yielding

$$
V_{2}-V_{2} \times \Omega+V_{1}+V_{1} \times \Omega=2 V^{\prime}
$$

Rearranging, we have

$$
V_{2}+V_{1}-\left(V_{2}-V_{1}\right) \times \Omega=2 V^{\prime}
$$


Taking the cross product from the right with $\Omega$, gives

$$
\left(V_{2}+V_{1}\right) \times \Omega-\left[\left(V_{2}-V_{1}\right) \times \Omega\right] \times \Omega=2 V^{\prime} \times \Omega
$$

The first term on the left reduces to $\left(V_{2}-V_{1}\right)$ using eqn. (2). The second term reduces to

$$
\left[\Omega \cdot\left(V_{2}-V_{1}\right)\right] \Omega-\Omega^{2}\left(V_{2}-V_{1}\right) .
$$

We know, again from eqn. (2), that $\Omega$ is perpendicular to $\left(V_{2}-V_{1}\right)$ so the first term in this expression vanishes. Eqn. 7 then reduces to

$$
\left(1+\Omega^{2}\right)\left(V_{2}-V_{1}\right)=2 V^{\prime} \times \Omega .
$$

The net result of this manipulation is that eqn. (2) that describes particle rotation can be replaced by the two equations

$$
V^{\prime}=V_{1}+V_{1} \times \Omega
$$

and

$$
V_{2}=V_{1}+\frac{2}{\left(1+\Omega^{2}\right)} V^{\prime} \times \Omega
$$

The net result is that we may move the particles with time-centered implicit pushes using eqns. (1), (9a), (9b), and (3). If care is taken with precisely what one uses for $B_{n}$, it is possible to obtain a pusher that will provide the correct cyclotron frequency regardless of DT. We do not keep the extra terms (of order $\mathrm{DT}^{3}$ ) but details can be found in Hockney and Eastwood or Birdsall and Langdon.

The transformation of $\mathrm{Vx}$ and $\mathrm{Vy}$ into $\mathrm{Vr}$ and $\mathrm{Vq}$ is accomplished by

$$
\begin{aligned}
& r(1)=\operatorname{sqrt}(x * x+d y * d y) \\
& \operatorname{costh}=x / r(1) \quad \text { sinth }=d y / r(1) \\
& v x(1)=\cos t h * v x h+\operatorname{sinth} * v y h \\
& v y(1)=-\operatorname{sinth} * v x h+\cos t h * v y h
\end{aligned}
$$$$
\text { THROUGHOUT GYMNOS, we use VX }
$$$$
\text { for } V R \text { and } V Y \text { for } V Q \text {. }
$$

where the generation of the new $\mathrm{R}$ from the new $\mathrm{X}$ and $\mathrm{Y}$ is obvious. [We find it convenient to use the variable $\mathrm{Vx}$ for $\mathrm{Vr}$ and $\mathrm{Vy}$ for $\mathrm{Vq}$ for ease of storage although on occasion in PARMOV, they actually contain the Carteasian components.] With axisymmetry in 2.5D, no loss of generality is experienced if the particle is assumed to be back at $\theta=0$ for the start of the next time step-so long as the correct value for $\mathrm{Vq}$ is maintained.

The remaining physics part of PARMOV is a straightforward reapplication of these steps for the first-order extrapolation of velocities at time $t_{n+1 / 2}$ (that will be stored) to time level $n+1$ (which will not be stored) for use in the "raw count" source accumulation.

Notice that PARMOV runs one particle at a time through this loop for the entire buffer load set up by TRANS. No thought is given, in this version, to vectorization or optimization(see sections discussing VPARMOV). For the new user of this basic code, loss of readability and flexibility cause more wasted CPU cycles than are usually recouped using clever coding. 
We have accumulated "raw counts" in each cell. Returning to TRANS after all particle buffers have been processed, we divide each particle source term by the volume associated with that cell radius. The density DEN, for example, returned to TRANS from PARMOV contains a simulation particle count in each cell. When a species is finished, DEN(I,J,N) will be divided by the volume of the computational cell EFFVOL that turns DEN into a number density of simulation particles in each cell and is ultimately converted to a real plasma density by multiplying by the number of real particles/simulation particle RPPSP. (see discussion of TRANS, Sec. III.A.4.a)

\section{IV.A.2.b) TRAP of the Particles Outside Plasma Region}

\section{IV.A.2.b.1) Processing LOST PARTICLES}

At the start of a time step, we know all particles are in legitimate simulation regions. Particles are moved to their $n+1$ location in the top of PARMOV. The call to TRAP after this time step checks each of these new positions to see if they have moved into a prohibited region. TRAP accomplishes this by looking at the cell center indices the particle now has

c

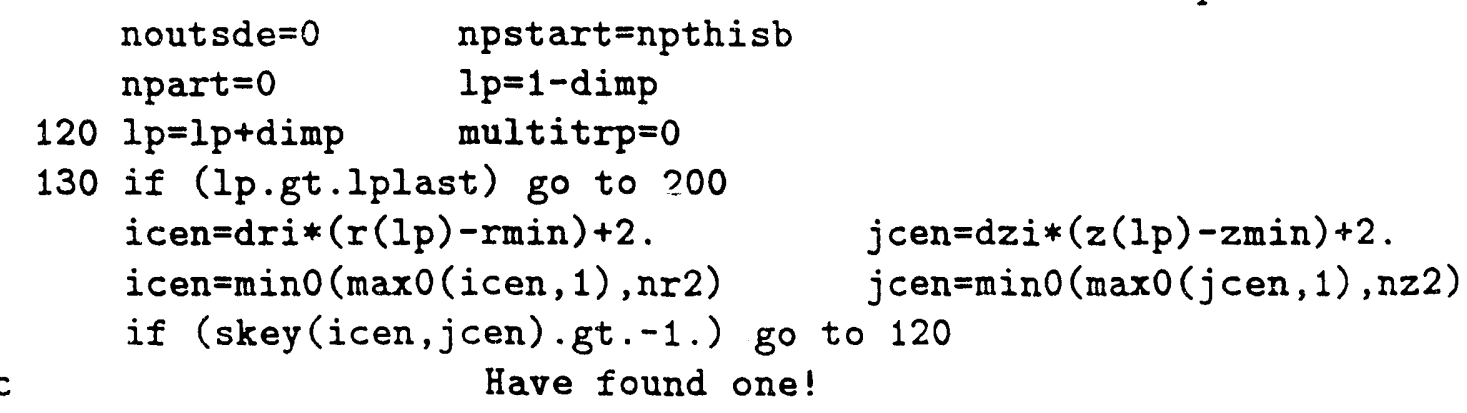

and looking at the value of SKEY with those same indices. SKEY(ICEN,JCEN) will be -1 . if the volume represented by these indices is inside a structure. Even particles that move well outside the simulation region will have ICEN,JCEN moved back to the mesh edges via the MIN0,MAX0 functions and these boundaries are always structures of some kind. (Unless the boundary is periodic-see details in the discussion of structures in GEOSET in subroutine BCSET.)

\section{IV.A.2.b.1.a) Determining Closest Corner}

When we find a particle "outside", we next determine its CFL number (the number of cells through which it is now moving per $\Delta t$ ) in each direction. We then cycle back with smaller time steps DTCLS (such that CFL $<1$ ) if necessary to find where last particle/boundary crossing occured. TRAP may use multiple passes through this procedure to catch those whose reflection puts them in yet another structure. The coding looks like this.

c

Have found one!

$\operatorname{pcflr}=a b s(d r i * v x(l p) * d t)$ 


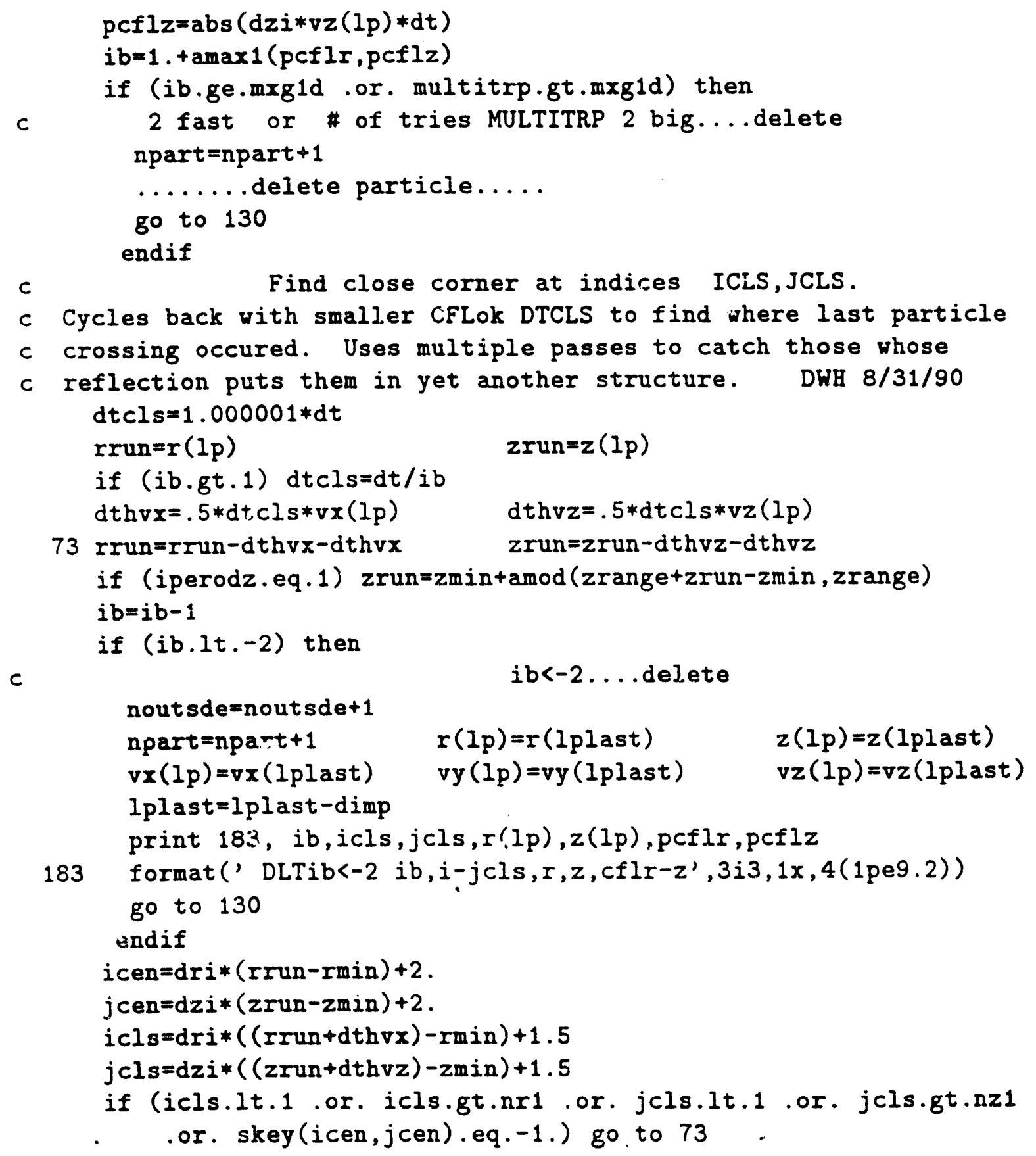

The purpose of this procedure is to find the indices of the corner closest to the place where the particle crossed into a forbidden structure. Armed with the indices of the "closest corner" ICLS,JCLS, the code then interegates the array IPBC to determine what to do with the offending particle.

\section{IV.A.2.b.1.b) Perfect Reflection or Delete}

Since the particles outside the periodic dimensions have already been returned, it is the 
physics properties of the closest corner determine which of two remaining courses of action will be followed. 1) If the closest corner is a point on a perfectly reflecting surface (denoted by IPBC(ICLS,JCLS)=51), the particle is perfectly reflected. If not then 2) the particle is logged and deleted by overwriting this particle with the last particle in the current buffer. All processes other than perfect reflection involve absorption and reemission.

Perfect reflection involves first determining the "orientation" of the surface associated with the closest corner ICLS,JCLS. The orientation is found in the array KORNT that has been set in routine GEOMSET using SKEY data. The best documentation is already contained in TRAP comment cards that are reproduced here. 
.

c

c

c

C

c

c

c

$c$

C

c

C

c

c

c

c

C

c

c

C

c

c

c

c

c

ok PERFECT REFLECTION-orient the surface with KORNT

$R$-reflect if

$(R(L P)-R G(I C L S)) * V X \quad>=0$

and

KORNT * $(\mid$ KORNT $\mid-10) * V X<0$

or

$\mid$ KORNT $\mid=1$

Z-reflect if

$(Z(L P)-Z G(J C L S)) * V Z \quad>=0$

and

KORNT* $(\mid$ KORNT $\mid-1) * \mathrm{VZ}<0$

or

$\mid$ KORNT $\mid=10$

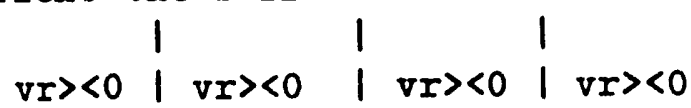

$\mathrm{vz}><0|\mathrm{vz}<0| \mathrm{vz}<0|\mathrm{vz}\rangle<0$

vr>0 $|8 / / / / / 10 / / / / 2|$ vr $<0$

vz $><0 \mid / / / / / / / / / / / / /$ vz $><0$ $1 / / / / / / / / 1 / / / / / / 1$

$------+-1 \quad---$ KORNT--- 1+--------

$1 / / / / / / / / / / / / / / / 1$

vr>0 $\mid / / / / / / / / / / / / / / /$ vr<0

vz $><0|-12 / /-10 / / \quad-8| v z><0$

vr $><0 \mid$ vr $><0 \mid$ | $r r><0 \mid$ vr $><0$

$v z><0|v z>0| v z>0 \mid v z><0$

Besides the obvious conditions of |KORNT|=1 or 10 for the plain sides, we use two types of conditions for reflection. The first depends of a product of velocity and position as

$$
\begin{array}{r}
(R(L P)-R G(I C L S))>0 \text { so that } V R>0 \text { r-reflects } \\
<0 \text { so that } V R<0 \text { r-reflects } \\
(Z(L P)-Z G(J C L S))>0 \text { so that } V Z<0 \text {-reflects } \\
<0 \text { so that } V Z>0 \text { z-reflects }
\end{array}
$$

The second condition depends on corner orientation KORNT $*(\mid$ KORNT $\mid-10)<0$ for $8,9,-11,-12$ so only VR>0 r-reflects $>0^{\circ}$ for $11,12,-8,-9$ so only VR<0 r-reflects

KORNT*( $\mid$ KORNT $\mid-1)<0$ for $-11,-12,-8,-9$ so only VZ>0 $z$-reflects $>0$ for $11,12,8,9$ so only VZ<0 $z$-reflects

Either the velocity-position or the KORNT conditions will suffice for outside corners because troublesome locations are not "inside" boundaries. To do the inside corner correctly for all cases requires both conditions to be satisfied. Far example, we want no z-reflection from inside +9 corners for particles in upperright quadrant, when incident from upperleft with vr>0 and vz>0. Without the KORNT condition it will z-reflect. Similarly, we want no $z$-reflection from inside +9 corners for particles in upperright quadrant when incident from upperleft with $v r>0$ and $v z<0$. In this case KORNT will reflect but $x-v$ condition will not.

flect $2=0$.

ikornt $=i a b s(k \circ r n t(i c l s, j c l s))$ 


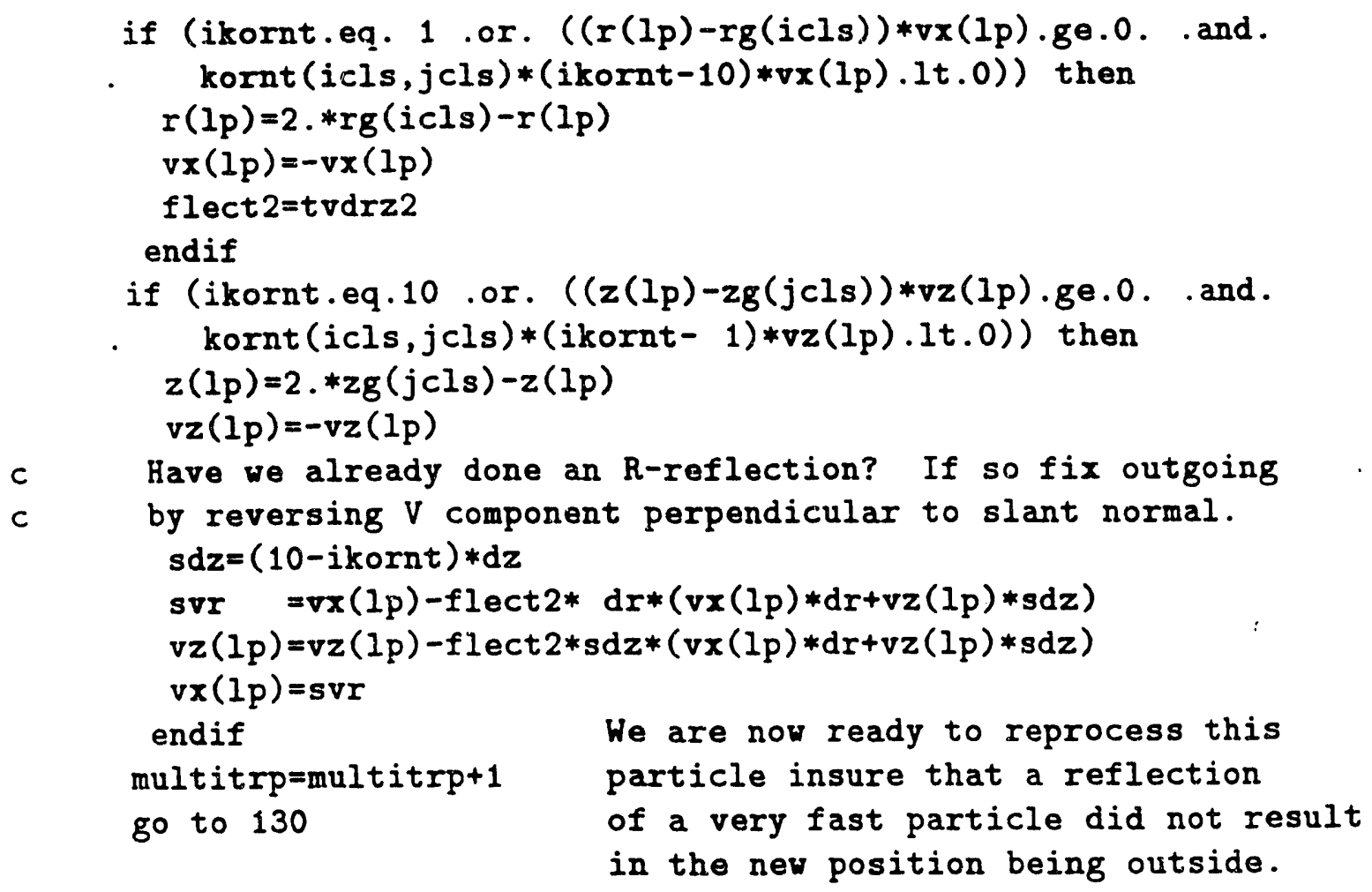

After some bookkeeping to account for all the particles lost, we are ready to consider thermal reemission or, if there is room in this buffer, injection or field emission.

\section{IV.A.2.b.2) Thermal Reemission}

If any particles have been deleted in previous operation in TRAP, we will have kept track of the total charge absorbed in each corner ICLS,JCLS in array QABSRB. (If an closest corner was unable to be identified for some reason, the particle's charge is simply lost.)

\section{IV.A.2.b.2.a) Controls from PBCSET}

c QABSRB (ICOR, JCOR) is the charge absorbed by cell corner ICOR,JCOR

$c$ Thermal reemission uses QABSRB as the resevoir. Reemit

c .02*|IPBC|*QABSRB/SQ using \#s in NSTPROP.

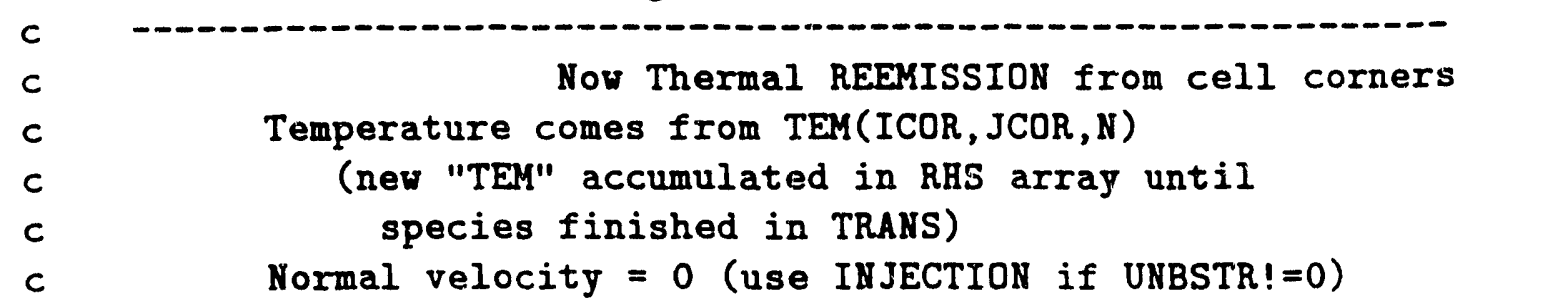


The controls for thermal reemission are set in PBCSET by a call before processing on the present species was begun. The primary control array for all structures is NSTPROP(NSTR,N). This array is used to determine IPBC(ICOR,JCOR) for each corner from the definition in PBCSET

$$
\begin{aligned}
& \text { nbtyp=iabs (nstprop (nstr, } n)) / 100 \\
& \text { nbpct }=i a b s \text { (nstprop (nstr, n)) }-100 * \text { nbtyp } \\
& 690 \\
& \text { ipbc }(k c i j(k c o r, 1), \operatorname{kcij}(k \operatorname{cor}, 2))=n b p c t
\end{aligned}
$$

and was discussed in section A2. Note that no normal velocity is allowed.

\section{IV.A.2.b.2.b) Mechanics}

TRAP begins the reemission process by cycling through the structures determine, first. if the structure allows reemission from the structure in question. If reemission is allowed. then the corner points of that structure are considered to see how many, if any, particles were absorbed at that corner and, finally, what fraction of those absorbed are allowed to be reemitted. If a corner passes all these tests, particles may then be given birth at this corner with all the additional specifications given by BEAM and NSTRPROP. The code in question follows

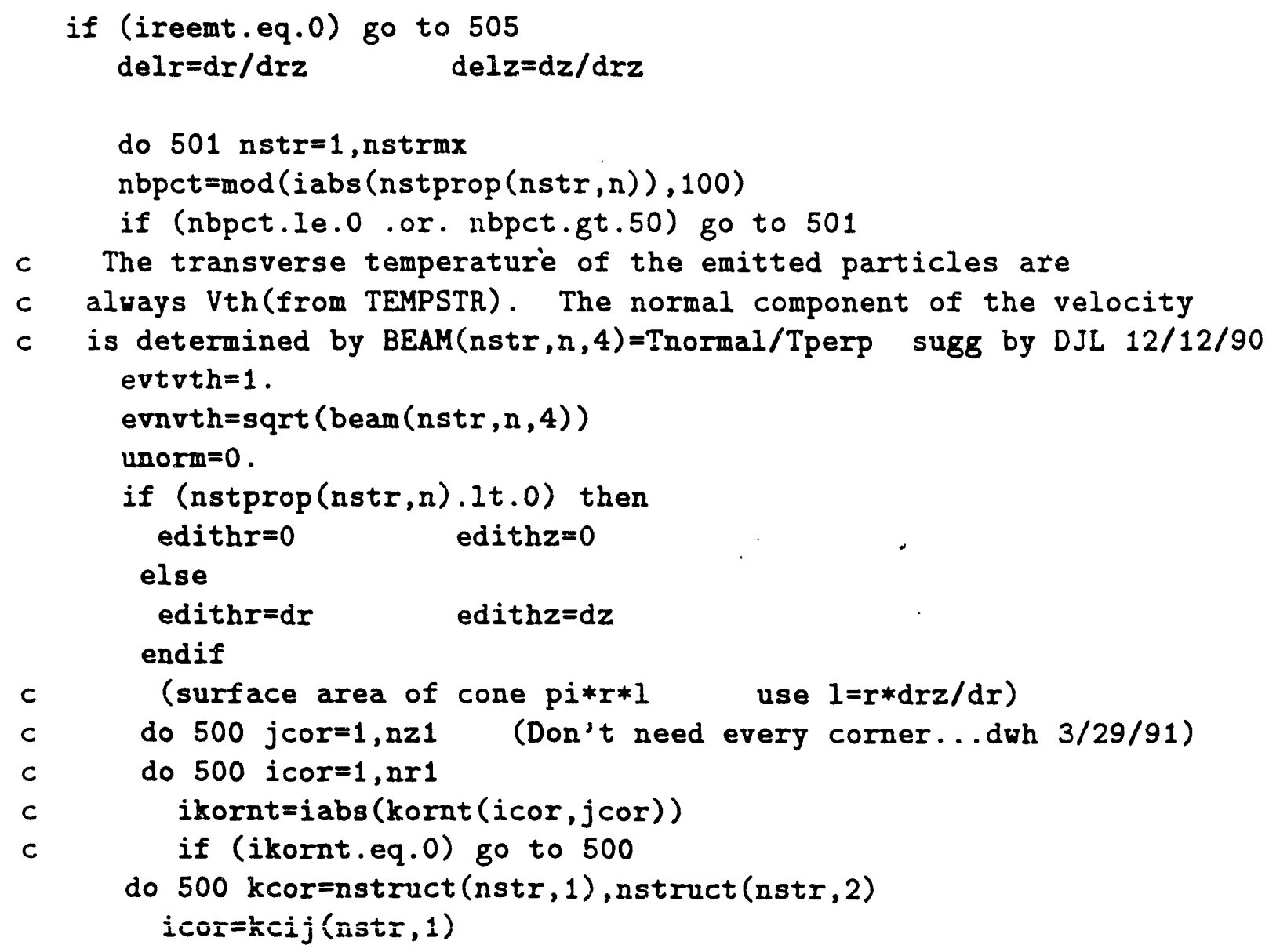




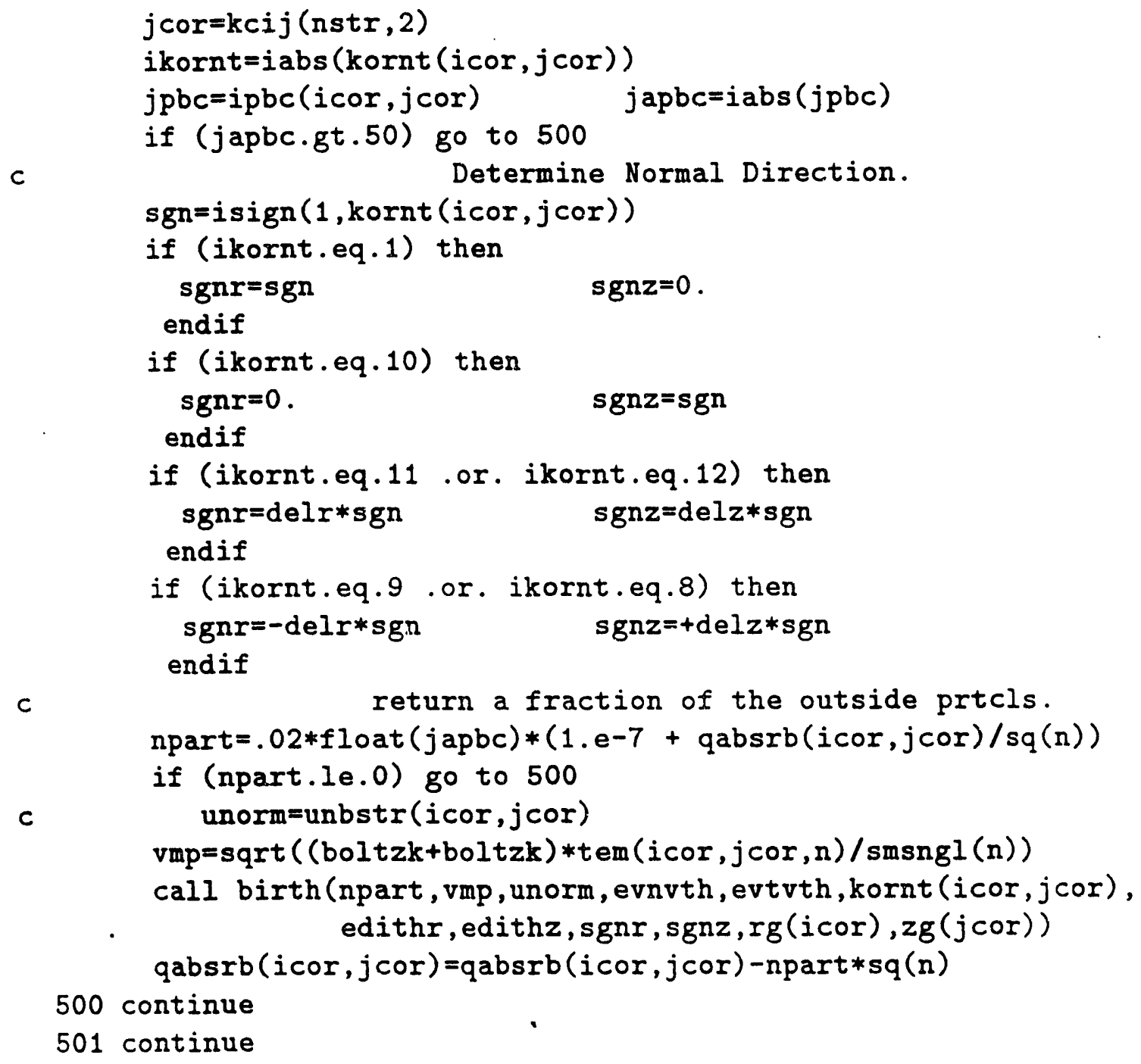

BIRTH obviously creates NPART particles with the other parameters in the argument list. Details are found in a appendix.

\section{IV.A.2.b.3) Injection and Field Emission}

In many ways similar to Thermal Reemission, the injection/filed emission section differs in that 1) absorption need not have taken place so that is reason to create new particles. 2) All that matters is that BEAM has a finite density to be emitted, that it is time for it to be "turned on" and that there is a structure that allows that process. Other significant differences are that non zero normal velocities are allowed, and that particles are created from cell faces [Left OR Down, LORD, means the face to the left of (or down from) the corner $\mathrm{KCOR}$ if $\mathrm{LORD}=1(2)]$.

\section{IV.A.2.b.3.a) Controls from PBCSET}

C QBRS(KCOR,LOID,N) is the resevoir for field emission and injection. 


\section{IV.A.2.b.3.b) Mechanics}

505 if (mpb.le.npthisb .or. dt.le.0.) return

if (npnjct(n).eq.0 and. npfemt(n).eq.0) return

C

NJECT and FLDEMT after advance before accumulation

Nnput $=0$

do 910 nstr $=1$, nstrmx

nbtyp=iabs (nstprop (nstr, n)) $/ 100$

if (nbtyp.eq.0) go to 910

nject $=$ nbtyp-1

if (nstprop(nstr, $n$ ).lt.0) then

edithr=0. $\quad$ edithz $=0$.

else

edithr=dr edithz=dz

endif

c The transverse temperature of the emitted particles are

c always vth(from TEMPSTR). The normal component of the velocity

$c$ is determined by $\operatorname{BEAM}(n s t r, n, 4)=$ Tnormal/Tperp sugg by $D J L 12 / 12 / 90$

evtrth=1.

evnvth=sqrt (beam (nstr, $n, 4)$ )

do 900 kcor=nstruct (nstr, 2), nstruct (nstr, 3)

c new stuff $3 / 20 / 90$ dwh

if $(q b r s(k \operatorname{cor}, 1, n) . e q .0$. and. qbrs $(k \operatorname{cor}, 2, n)$.eq.0.) go to 900 $i=\operatorname{kcij}(\operatorname{kcor}, 1) \quad j=k \operatorname{loj}(\operatorname{kcor}, 2)$

if ( $(i . e q .1$. and. j.eq.1) .or. $\operatorname{kornt}(i, j)$.eq.0 .or. kornt $(i ; j)$.eq.+11 or $\operatorname{kornt}(i ; j)$.eq.-12) go to 900 


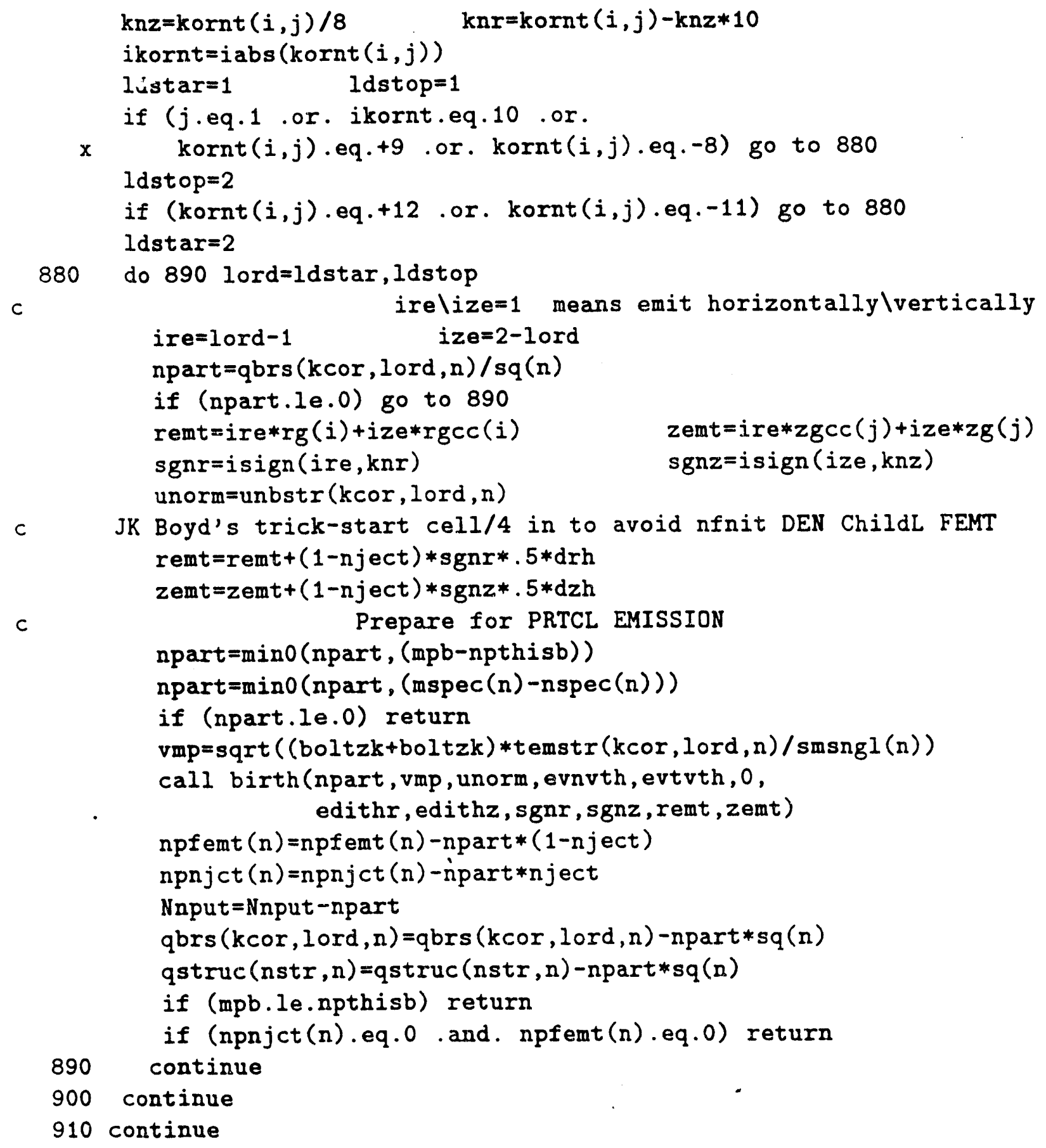

Further details about BIRTH can be found in an appendix. 
IV.A.3) Finish a Species, AUTOVLM, Restore E and B, DAPART Convert Raw Counts To Densities, Save Histories

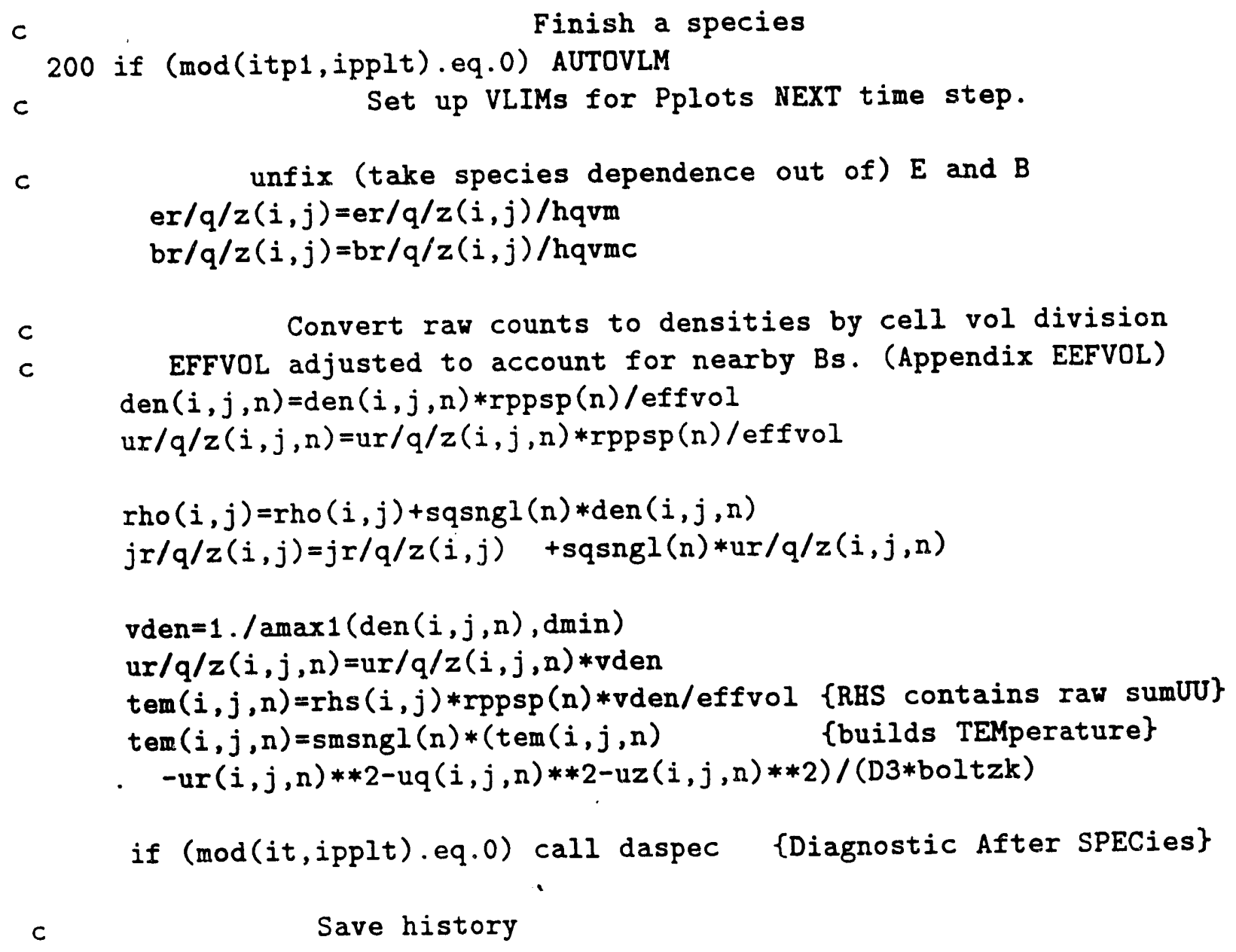

The commemt to save history is accomplished by a call to HISTORY and that rnutine is again documented in the DIAGNOS routine, subsection $F$.

\section{IV.B) Call DSOURCE (See VI. Diagnos)}

The diagnostics of the total plasma source terms summed over all species is carried out by the entry DSOURCE in the subroutine DIAGNOS. Details of this routine are given in Sec. VI.D

IV.C) Call FIELD-The The Director of the Field algorithm, using FLDSET (See V. BCSET)

The purpose of the subroutine FIELD is to provide the $E$ and $B$ fields needed for the next particle time step given the instanteous plasma source terms. Whether magnetostatic or Darwin, the field system is elliptic rather than hyperbolic so no previous field history are needed. Previous values make excellent 1 st guesses for iterative solvers. however. We 
compute $\mathrm{Ar}, \mathrm{Aq}, \mathrm{Az}$ and $\Phi$ using the latest set of source terms. The needed B fields are then computed from the curl of $A$.

The variable IWHEN has no physics or FORTRAN purpose: should the code die, a check of IWHEN with a debug routine such as DBX reveals the section of FIELD quickly.

The three components of $A$ and $\Phi$ are computed from Poisson-type elliptic equations. The $\mathrm{r}$ and $\theta$ component of $\mathrm{A}$ are in fact derived from the curl curl operator. The most important requirement, given the existence of VDKR is to have the boundary conditions arrays KEY, KN, BV, BVR, and BVZ properly set. This is taken care of in FLDSET that is called using appropriate arguments before each call to VDKR.

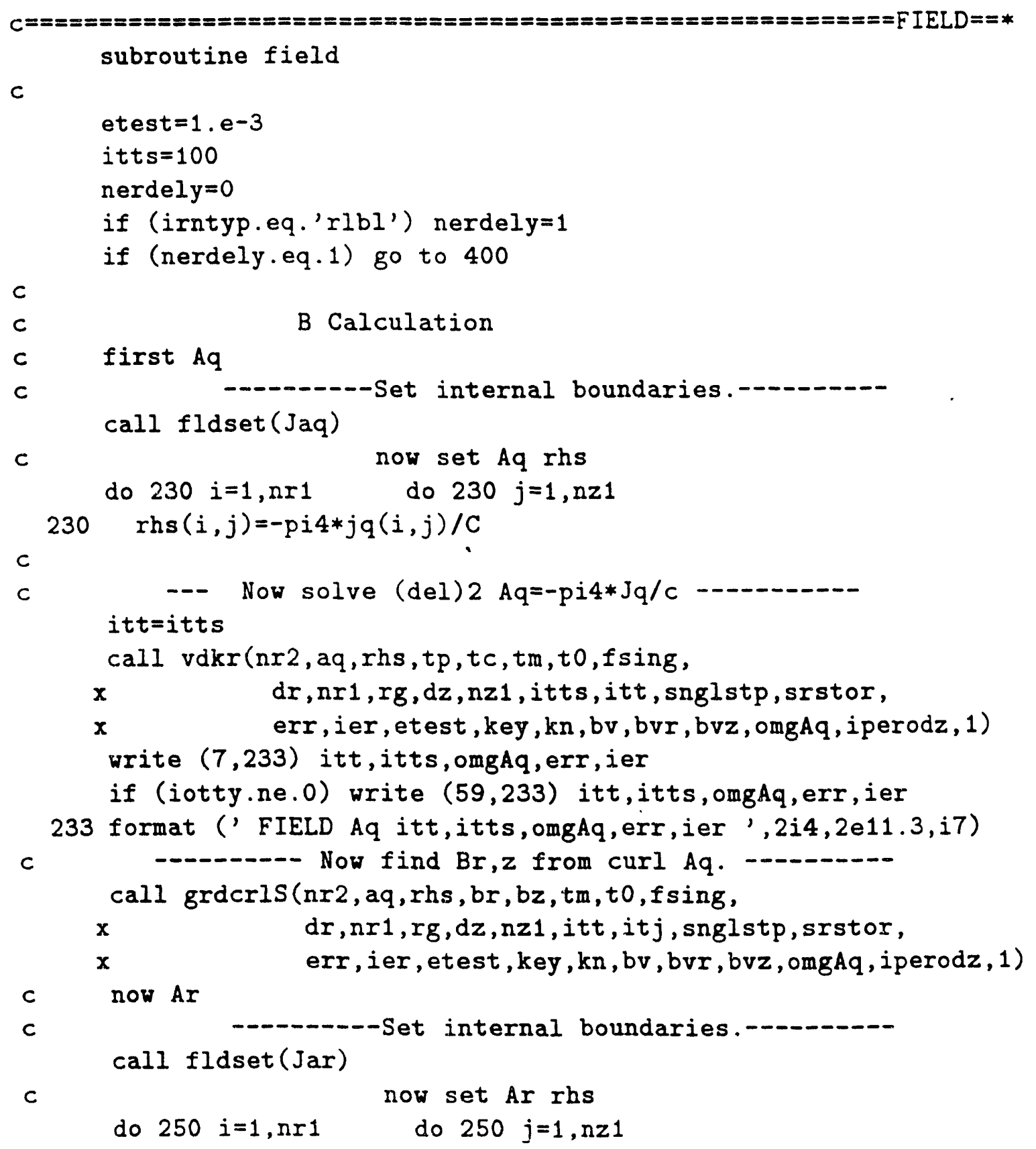




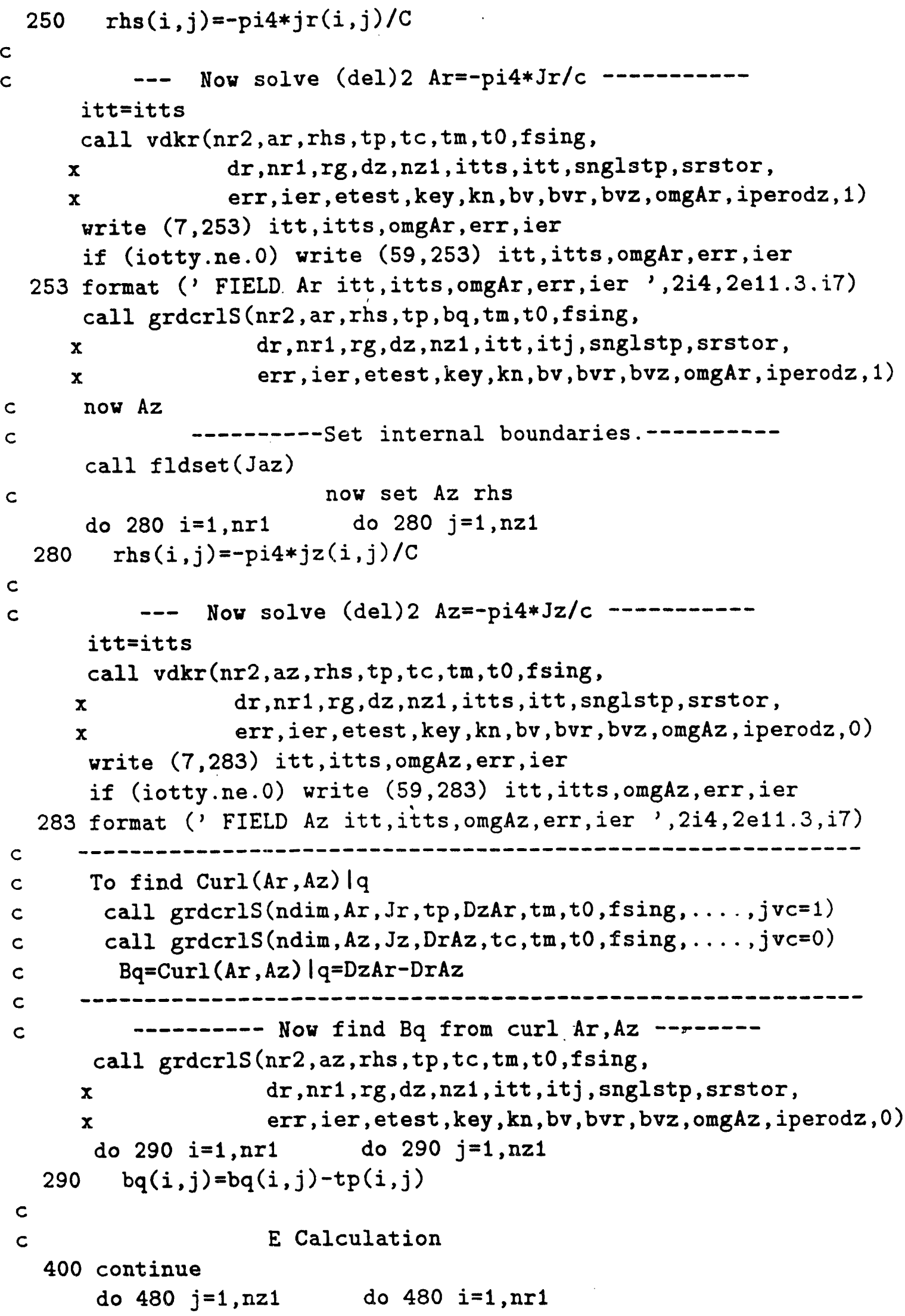




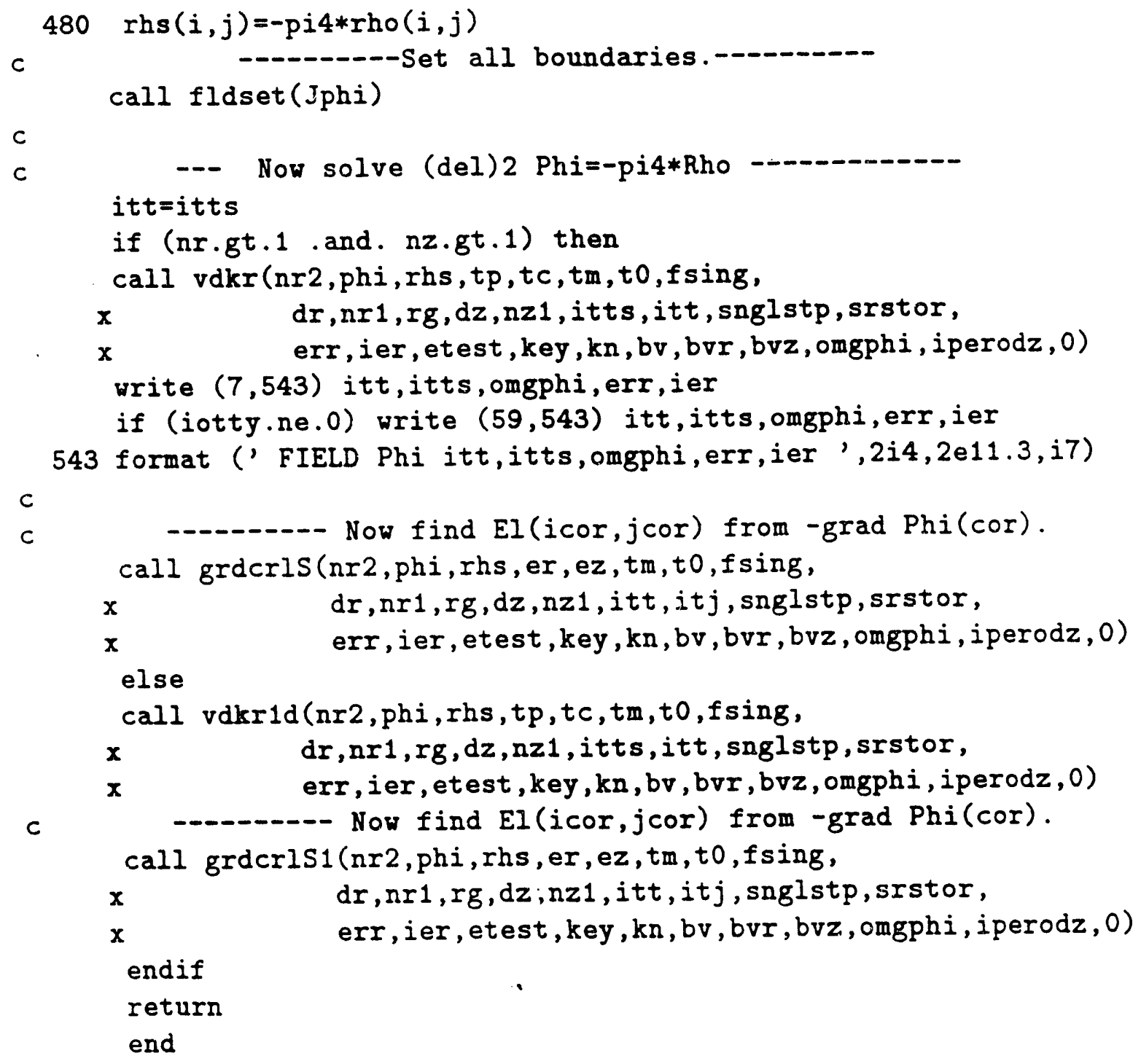

\section{IV.D) Call DFIELD (See VI. DIAGNOS)}

The fields are diagnosed and plotted in a straightforward way in DFIELD. Details are found in Sec. VI.E. 


\section{V) User Specified Physics Routines}

\section{V.A) Macroscopic Initial Profile Generation by INPROF}

For each species, INIT calls the routine INPROF for every cell center. This is the place for the general user to build his/her personalized initial configuration. A few examples are given here.

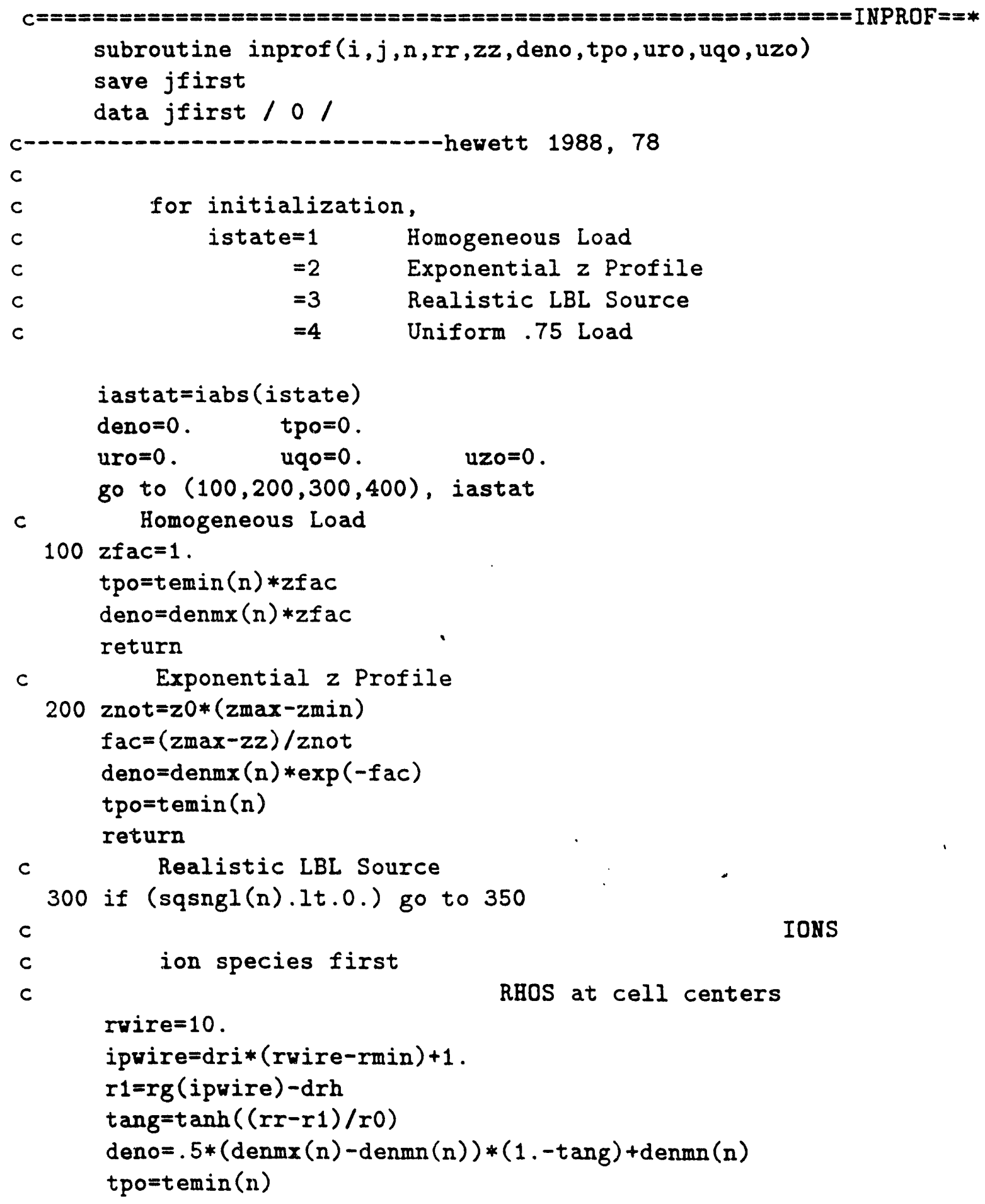




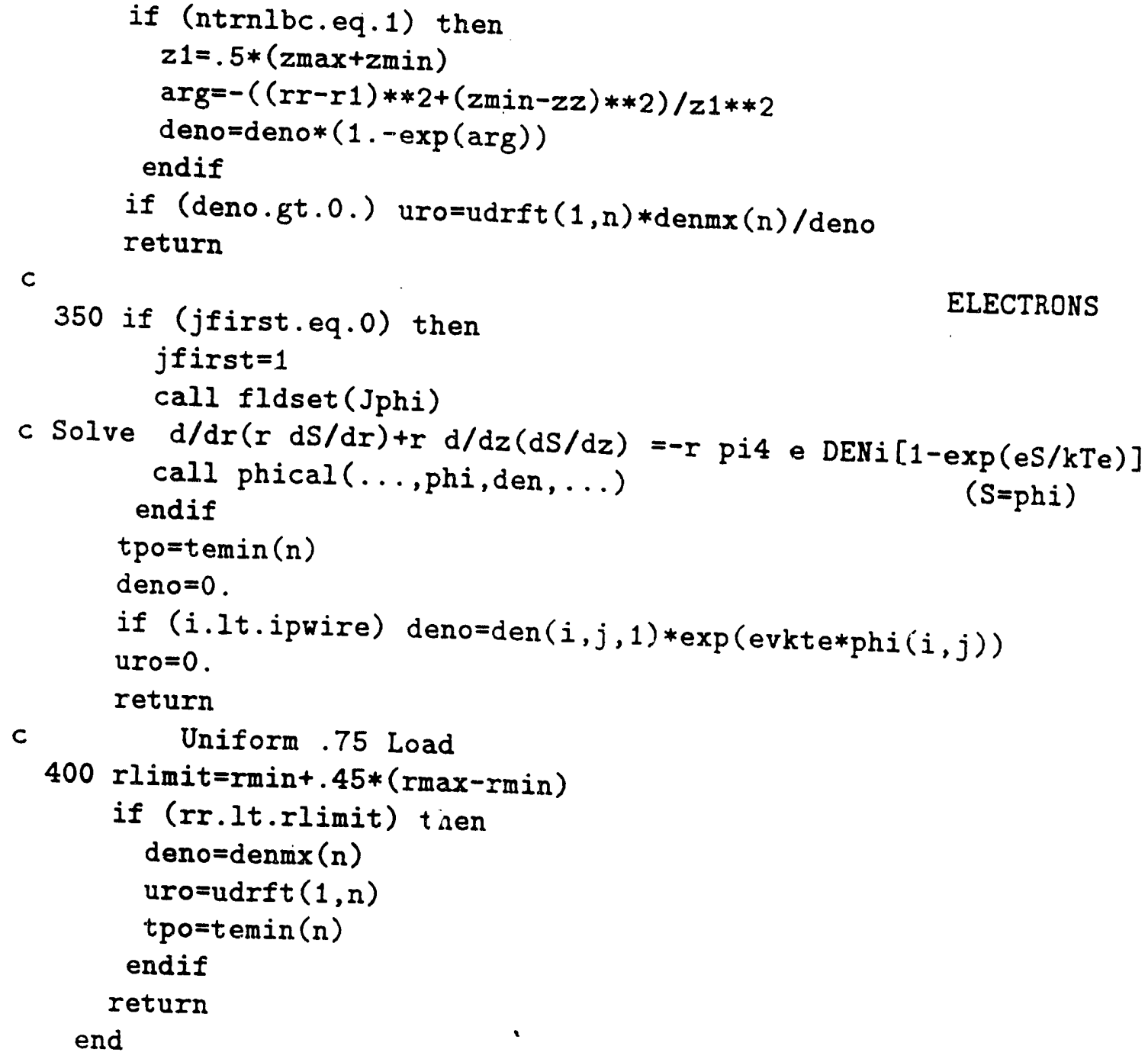

\section{V.B) User Specified Boundary/Structure Physics in BCSET}

BCSET is a general group of boundary conditions setting routines. They are quite separate in their functions but can be logically grouped within this one routine using several entries. The advantage is that the close proximity facilitates the physics interplay
that needs to occur between the various boundary requirements.

\section{V.B.1) GEOSET}

This entry is called very early in the run for the purpose of specifying the boundary in internal structures. It is here that the arrays STRGEN and NSTPROP are set thus determining the physical geometry of the simulation. Presumably, this routine could be
called during the run if we want a moving structure. 


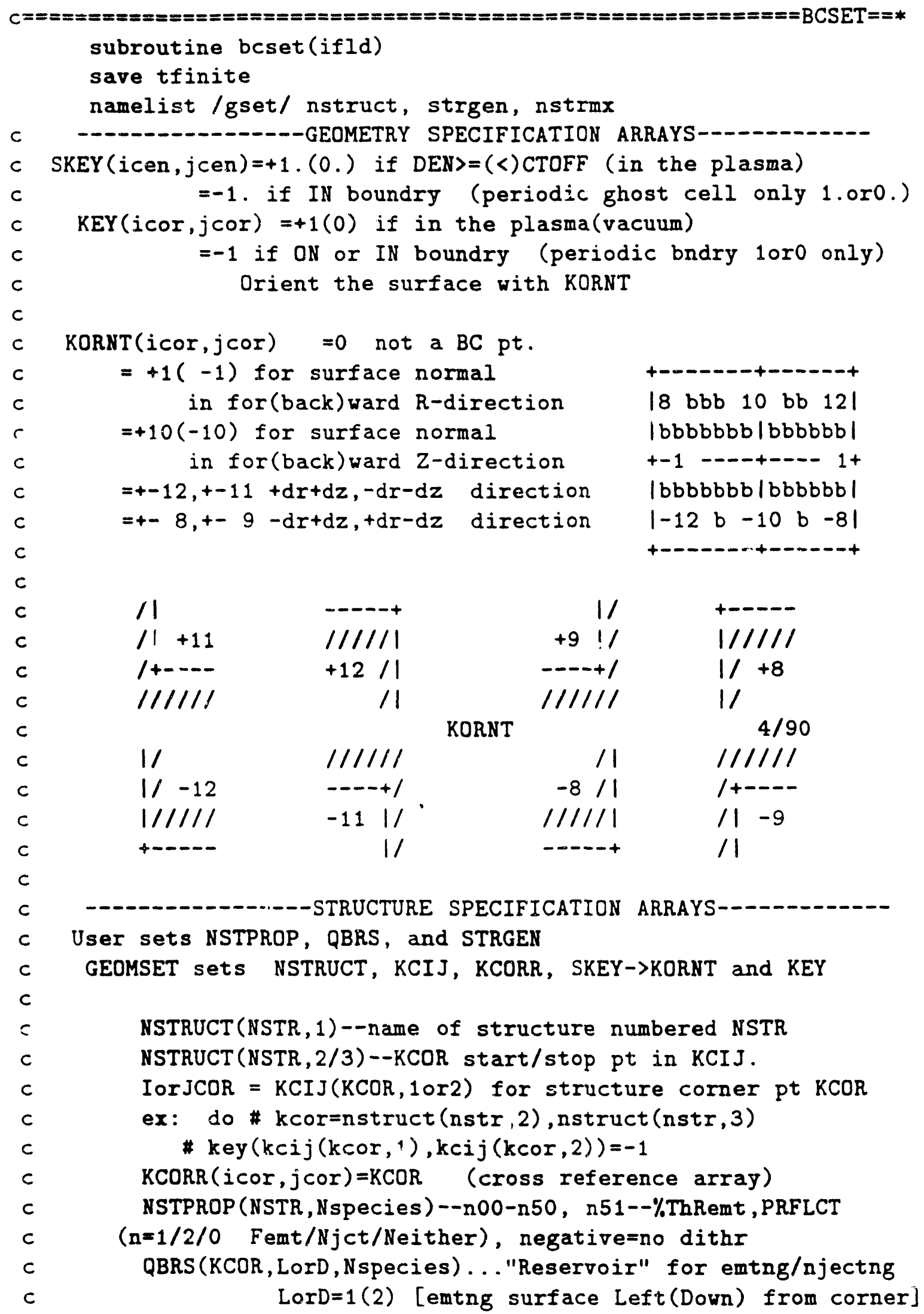


c

c

C

C

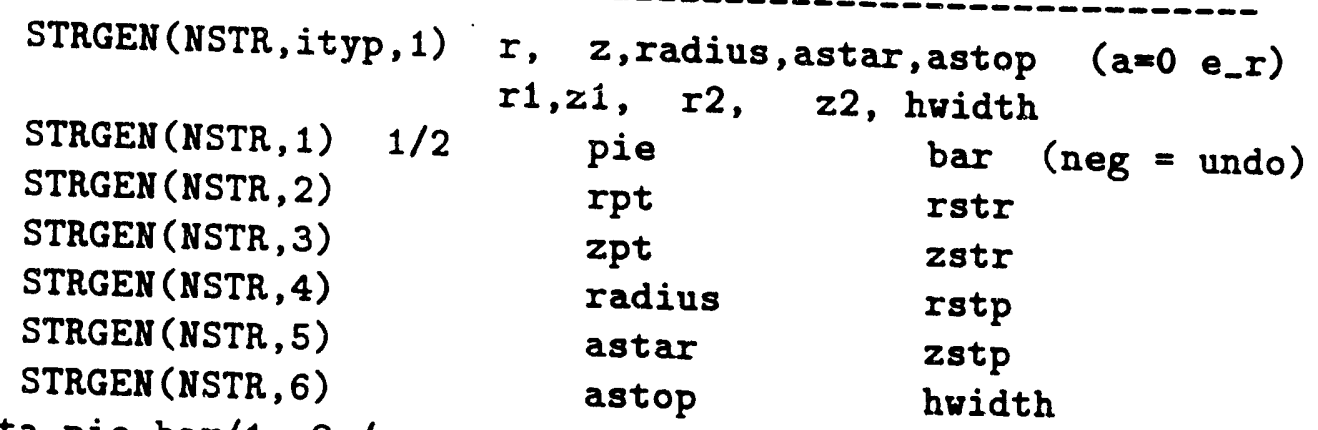




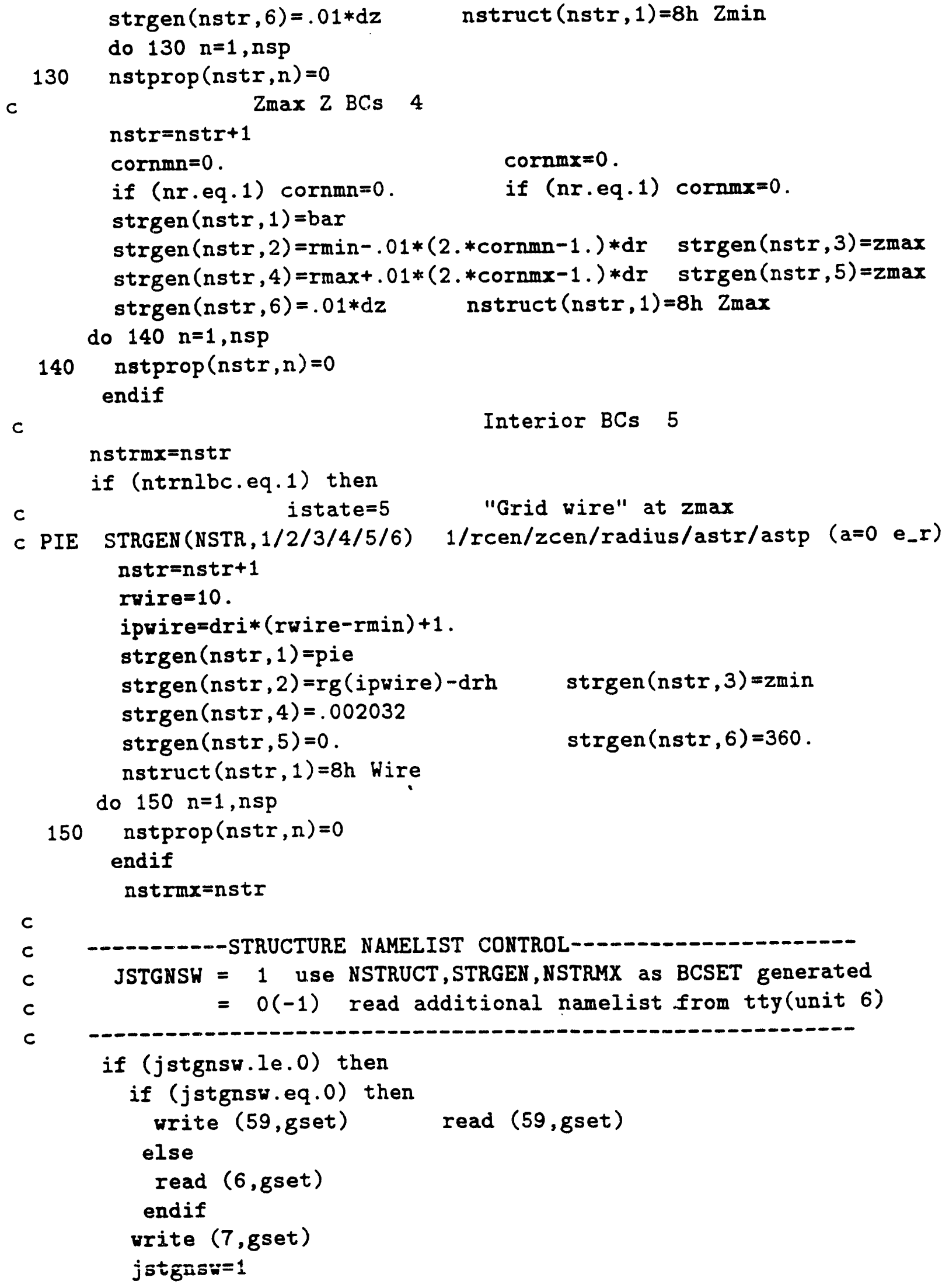




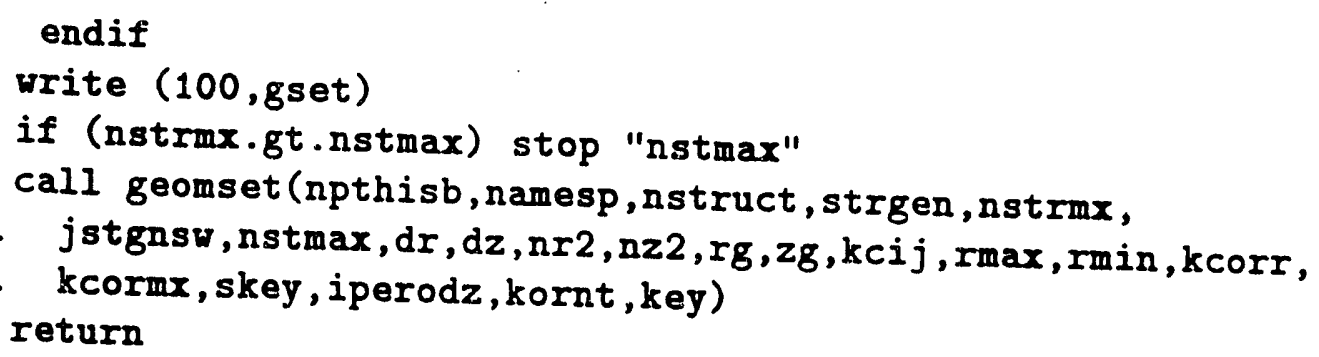

\section{V.B.2) PBCSET}

PBCSE'T is called as processing is initiated on each new species $N$ in TRANS. If, for example, we wish to field emit electron, TRANS calls PBCSET with the latest E field before any electrons are pushed. PBCSET then computes the surface charge density that this field would induced at each corner on all surfaces that are to emit. This charge density is converted to a net charge by multiplying the induced desity by the surface area associated with that corner. This charge then loaded into the charge reservoir QBRS(KCOR,LORD,N) to be emitied from either the surface to the left LORD=1 or down $L O R D=2$ from the $K_{C O R}{ }^{t h}$ corner for this species $N$ (electrons in this example).

This particular version given here has two options for injection. We often use the run type IRNTYP as a flag to switch options so that the code can be used for several types of work at the same time. The case for IRNTYP $=$ ' $b x$ ch' (for Beam a X the CHamber) is a simple injection algorithm where the normal velocity, stored in UNBSTR(KCOR,LORD,N) is obtained from BEAM(NSTR,N,2) and net charge to be added to the reservoir, again QBRS(KCOR,LORD,N), is the beam amplitude BAMP (input from BEAM(NSTR,N,1) and suitably modified by the time modifiers also stored in BEAM(NSTR,N,5/6/7/8)] times the species charge SQSNGL(N) times the injection velocity UNBSTR(KCOR,LORD,N) times the area IRE*AREAR+IZE*AREAZ [modified for horizontal or vertial injection] times DT. This is just the charge that such a beam would have moved through the surface
in one time step.

The second option is a "DEMAND" injection option. We wish to inject only the particles that are needed to maintain a preselected density, again from BEAM(NSTR,N,1). The idea here is to compare the preselected density with the average density DENAV and add the needed charge to the reservoir to be injected.

Count are made of the number of new particles to be injected and some care is taken to insure there is enough room to accomodate all the particles needed to satisfy these injection/emission requirements. Finally IPBC is set for all corners in the simulation for
the species now to be pushed.

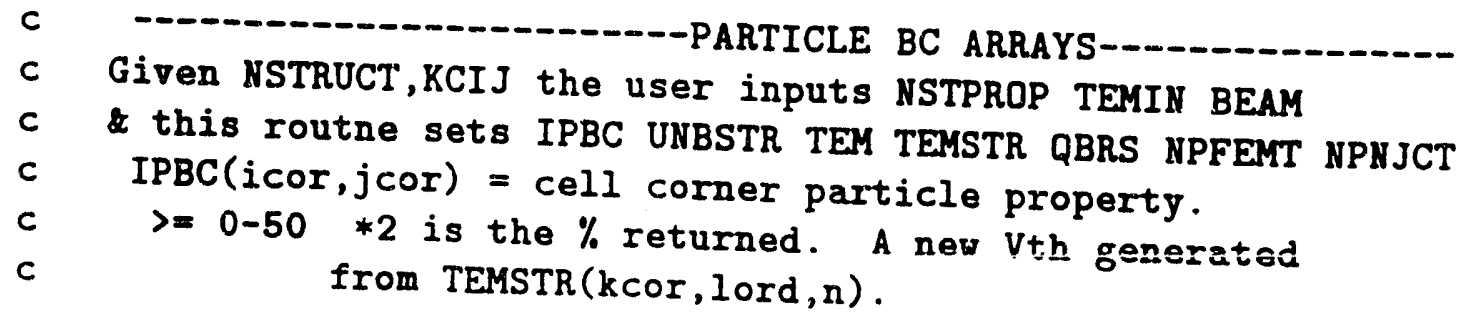




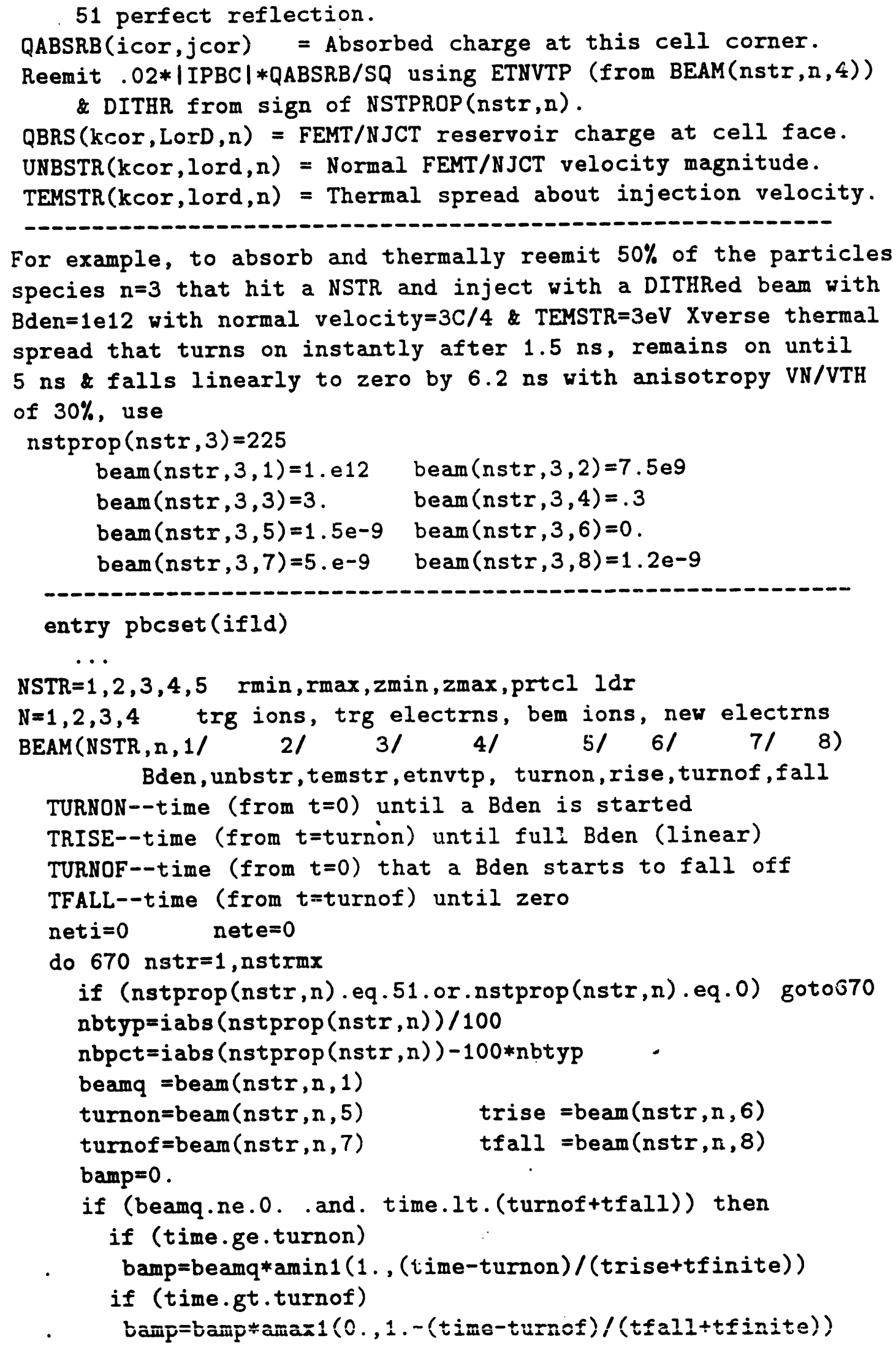




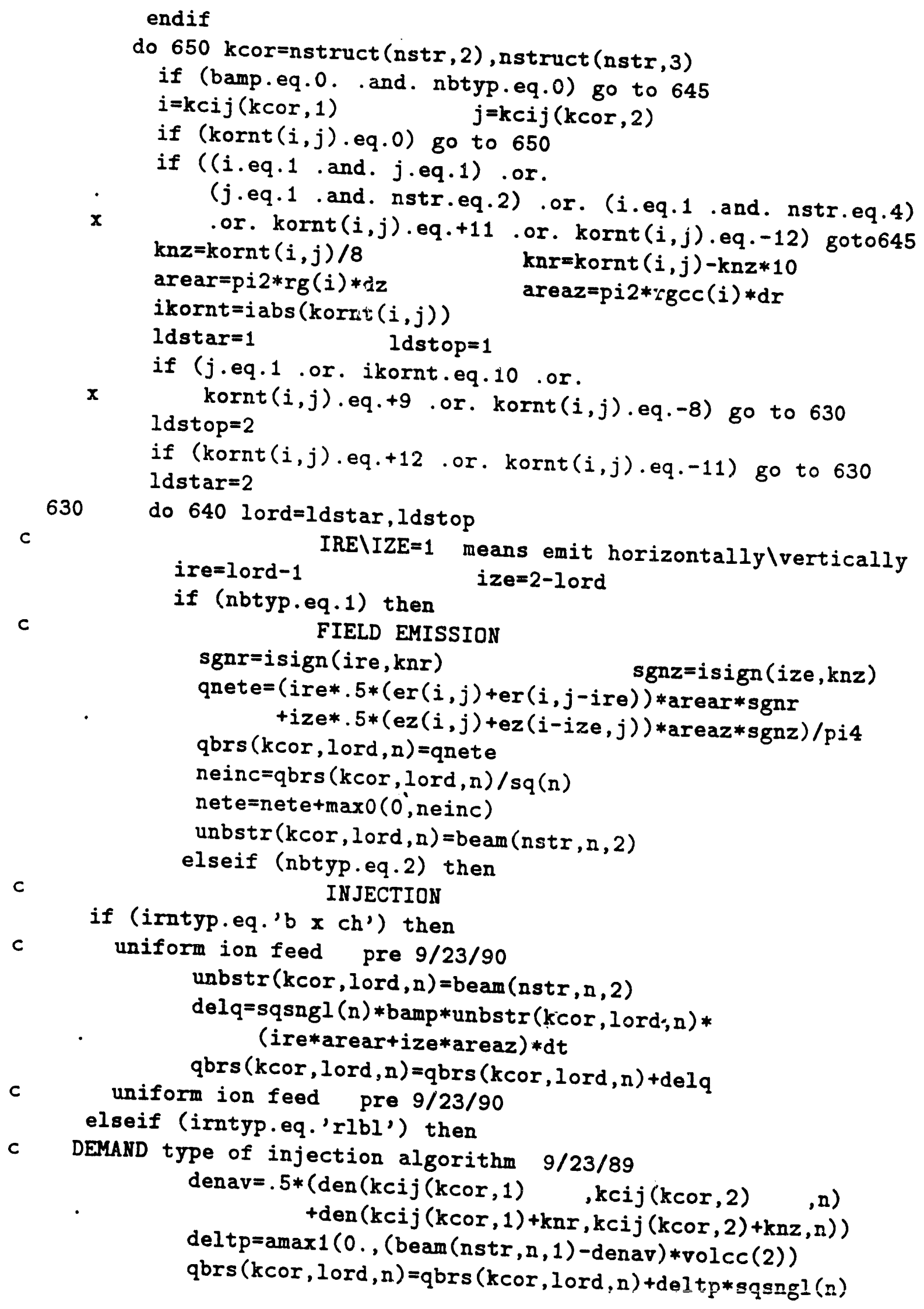




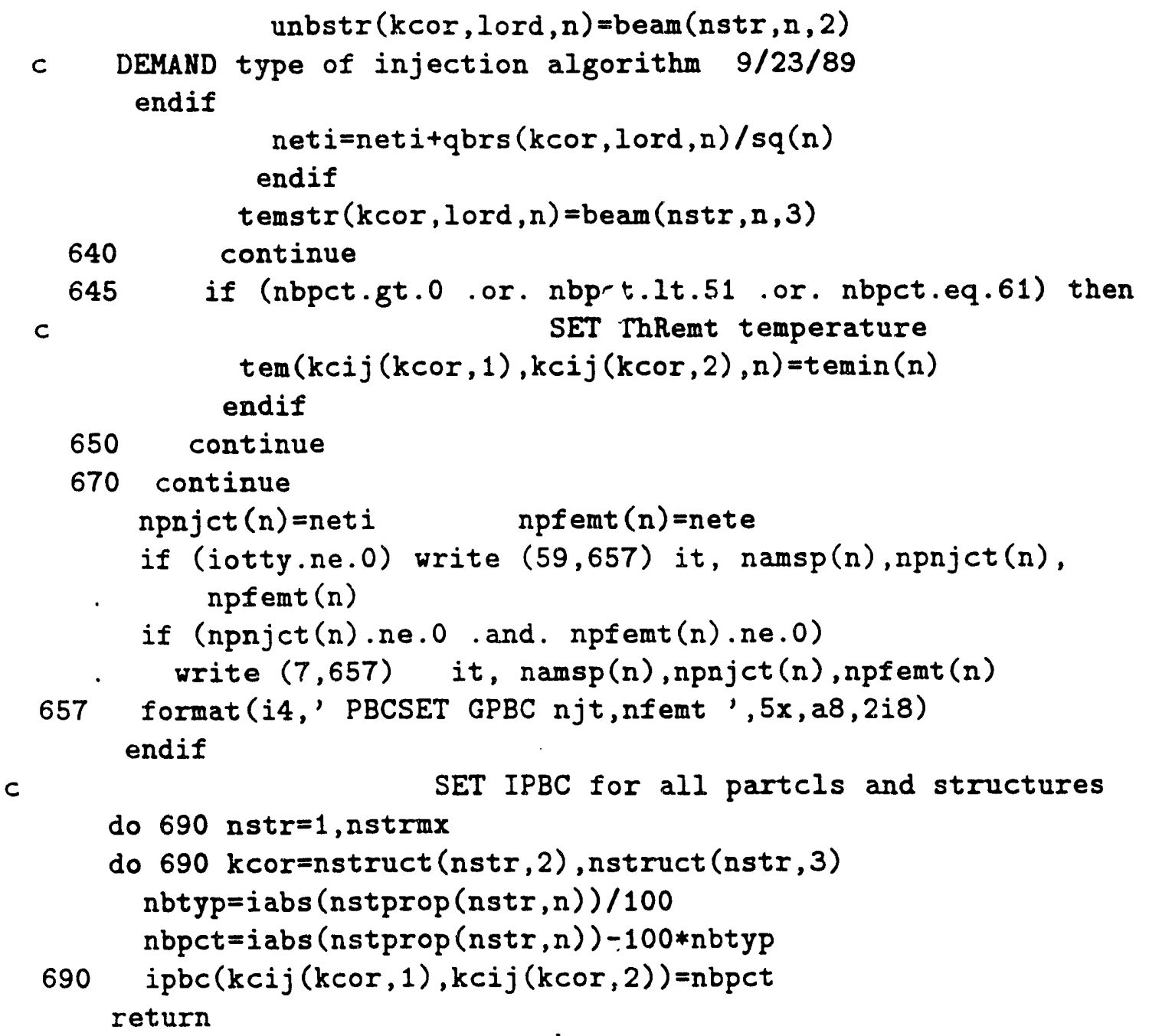

\section{V.B.3) FLDSET}

Here again we have used IRNTYP to switch between various types of runs. We also use the notation INN, IND, etc to express boundary conditions on various surfaces. The second character, either $\mathrm{N}$ for Neumann or $\mathrm{D}$ for Dirichlet, represents the boundary condition to be applied on the lower boundary and the third character represents the condition to be applied on the top boundary. IPD is also allowed and that gives a periodic condition. This stuff is mostly a holdover from earlier work and it is not as useful as in earlier codes that had no internal boundaries.

The coding below the statement label 2000 contains time dependent boundary condition provisions motivated by physics associated with external circuits. Though primitive at the moment, this section contains the ideas that surfaces connected to external voltage sources can be expected to control Dirichlet boundary conditions for the electrostatic potential and external voltages produce $\mathrm{E}$ fields that produce finite time derivatives in $\mathrm{A}$ the magnetic vector potential. The time derivitives can then determine a time dependent Dirichlet condition for A that ultimately shows up in a modified B field-just what external 
voltages are supposed to do.

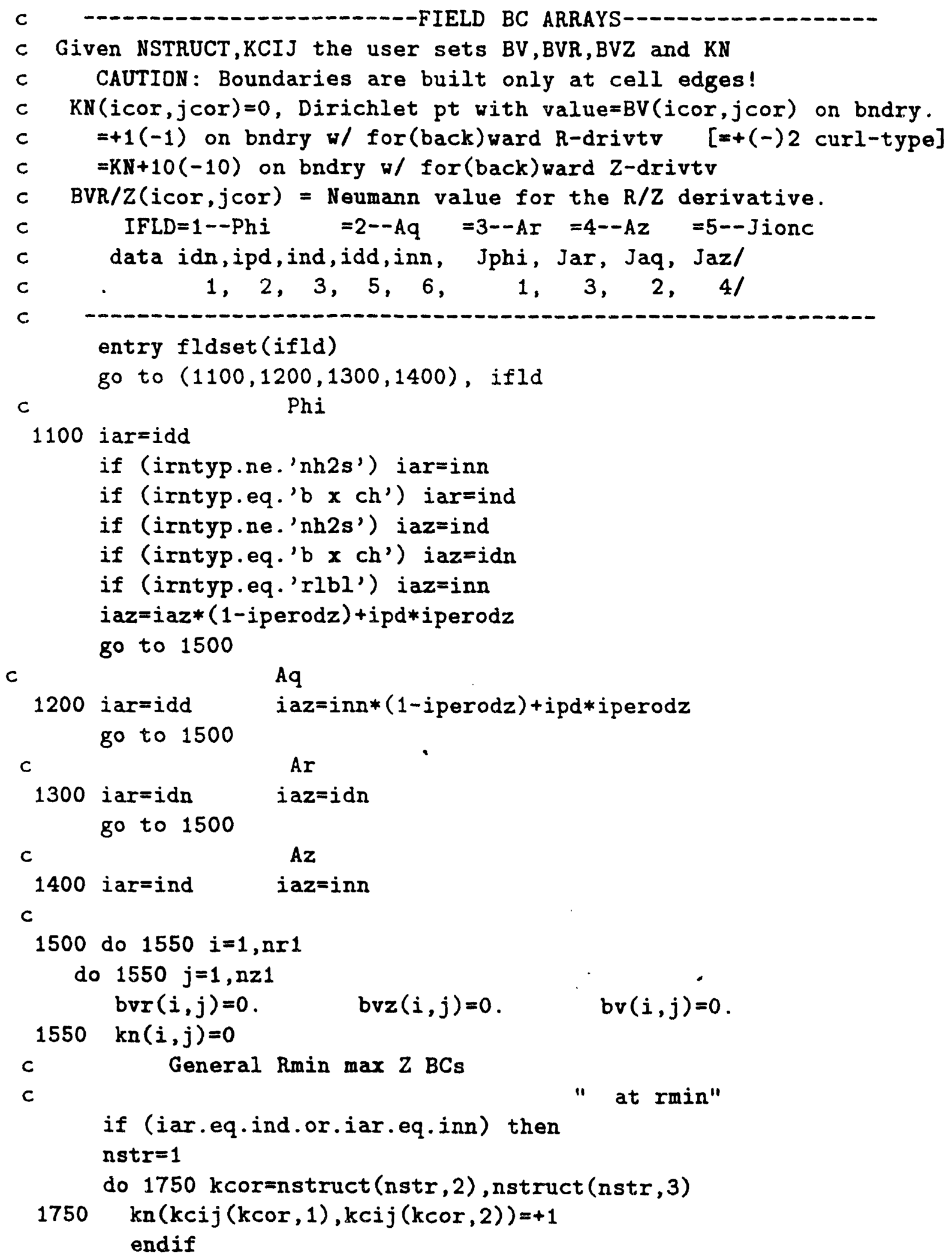


$c$

if (iar.eq.idn.or.iar.eq.inn) then

\section{nstr $=2$}

do 1755 kcor=nstruct (nstr, 2), nstruct (nstr , 3)

$1755 \operatorname{kn}(\operatorname{kcij}(k \operatorname{cor}, 1), \operatorname{kcij}(\operatorname{kcor}, 2))=-1$

endif

c

c

if (iperodz.eq.1) go to 2000

if (iaz.eq.ind.or.iaz.eq.inn) then nstr $=3$

do 1760 kcor=nstruct (nstr, 2), nstruct (nstr, 3)

$1760 \operatorname{kn}(\operatorname{kcij}(k \operatorname{cor}, 1), \operatorname{kcij}(\operatorname{kcor}, 2))=$

- $k n(k \subset i j(k \operatorname{cor}, 1), k c i j(k \operatorname{cor}, 2))+10$

endif

c

if (iaz.eq.idn.or.iaz.eq.inn) then

nstr $=4$

do 1765 kcor=nstruct (nstr ,2), nstruct (nstr, 3)

$1765 \operatorname{kn}(\operatorname{kcij}(k \operatorname{cor}, 1), \operatorname{kcij}(\operatorname{kcor}, 2))=$

endif

c

2000 continue

do $2010 i=1, n r 1$

do $2010 j=1, n z 1$

$\operatorname{bvr}(i, j)=0$.

$2010 \mathrm{bv}(i, j)=0$. $\operatorname{kn}(\operatorname{kcij}(k \operatorname{cor}, 1), \operatorname{kcij}(k \operatorname{cor}, 2))-10$

go to $(2100,2100,2300,2400)$, ifld

C

2100 if (time.le.tpsave) return

do 2180 nstr $=1$, nstrmx

c

c

c

C

c

c

Phi

\section{Voltq--increases NSTR flux with time} TURNON--time (from time=0) that a voltage is applied TFALL--time (from time=turnof) for linear falloff

PHISTR(NSTR, 0\&1\&2\&3\&4\&5) mag0, mag, turnon, rise, turnoff, foff TRISE--time (from time=turnon) until full voltage (linear) TURNOF--time (from time=0) that a voltage is turned off phibm $=$ phistr (nstr, 1$)$ turnon=phistr (nstr, 2) turnof =phistr (nstr , 4)

trise =phistr (nstr, 3 )

tfall =phistr (nstr, 5) if (phibm.ne.0. and. time.lt.(turnofttfall)) then phib=phibm*amax1 (0., amin1 (1., (time-turnon)/(trise+tfinite))) phib $=$ phib*amin1 $1 ., \operatorname{amax} 1(0 ., 1 .-($ time-turnof $) /($ tfall+tfinite $)))$ 


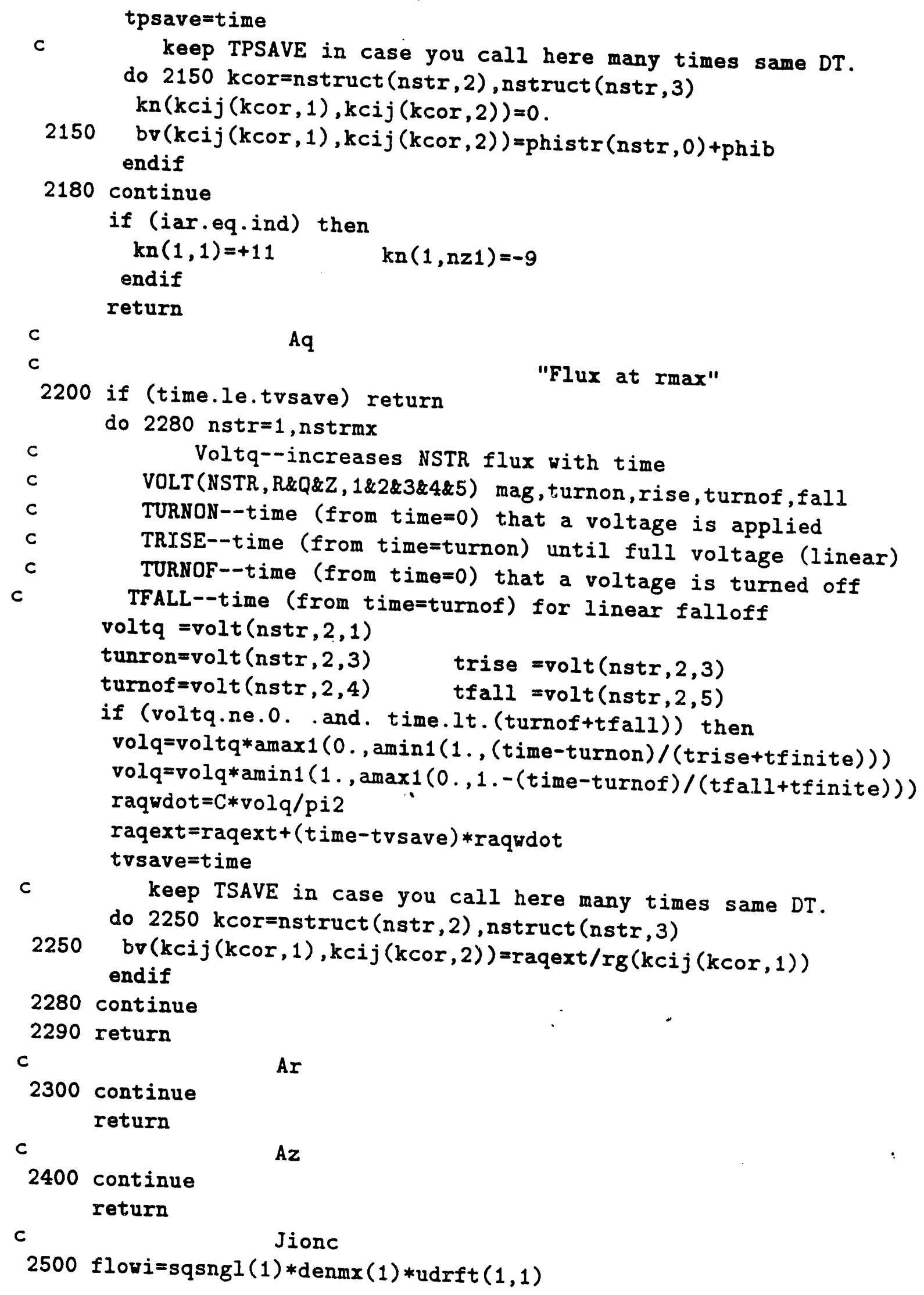




\section{nstr $=3$}

do 2320 kcor=nstruct (nstr, 2), nstruct (nstr, 3)

$2320 \operatorname{bvr}(k \operatorname{cij}(k \operatorname{cor}, 1), \operatorname{kcij}(k \operatorname{cor}, 2))=-$ flowi

c

$$
\text { do } 118 k=1, n r 1 \quad \operatorname{key}(k, 1)=-1
$$

$118 \operatorname{key}(k, n z 1)=-1$

do $119 k=1, n z 1 \quad \operatorname{key}(1, k)=-1$.

$119 \operatorname{key}(\mathrm{nr} 1, \mathrm{k})=-1$.

c

$$
\begin{array}{ll}
\operatorname{kn}(1,1)=+11 & \operatorname{bvr}(1,1)=-f \text { lowi } \\
\operatorname{kn}(1, n z 1)=-9 & \operatorname{bvr}(1, n z 1)=- \text { flow }
\end{array}
$$

return

end 


\section{VI) User Specified Diagnostics in DIAGNOS}

The intent of subroutine DIAGNOS is to provide a common location for all physics I/O for the code. However, diagnostics have much diversity and so in effect DIAGNOS is simply a grouping of these several types of independent diagnostic routines, each with its own entry point, under the one master subroutine name. DIAGNOS itself is never called. The discussion that follows is a guide to some of the diagnostics that have been found useful in the past. Although there are many places $i_{\perp}$ GYMNOS that the inexperienced user is advised NOT to make changes, the choice of diagnostics and how they are displayed do need strong input and involvement by the user. These examples should serve as a guide.

The details of the plot routines used here are given in the Appendix on graphics.

\section{VI.A) DPART Generates Particle Phase-Space Plots}

One of the reasons to run a PIC model rather than a fluid or MHD model is that the kinetic behavior of the particles is important. The most effective windows into this behavior are prtcl phase-space plots-point or "scatter" plots of individual particle velocities as functions of their coordinates. A good example is found in the VR vs. R plots of one of the ion source test cases that display clearly the kinetic ion behavior. The corresponding plot of VR vs. $Z$ would display the transverse position of the most active ions.

Since it is expensive to run through the particles if they are stored externally on disk. we try to take advantage of the buffering cycle that must occur to get the particles through PARMOV in the normal course of a time step. After a buffer load of particles has been processed, a call is made to DPART (Diagnostics on PARTicles) to see if this is a time step to do phase-space plots. If it is, the frames and scaling for these plots are set up on the first call and those particles that need to be plotted from the current buffer in core are plotted. Control is then returned to TRANS and the next buffer of particles is brought into core. After this next buffer is advanced in time in PARMOV, DPART is again called. This time the point plot PPLOT routines place points in the plot regions drawn previously.

One can see this logic, set up with the variable JFIRST, in the section DPART in routine DIAGNOS. We find it useful to put all six combinations on a given plot frame. To put them on separate frames would require either separate passes through the particle buffering routines (as occurs in DAPART below) or the ability to open up separate plot channels. A strong benefit of having all six phase-space plots on one frame is the correlation in space immediately available from the individual plots. What we are attempting to describe is a concept similar to a blueprint an object with the 3 standard views, front, top and side. We are arguing that it is useful to have all 3 views on the same piece of paper.

\section{VI.B) DAPART Generates Particle Position-Space Plots}

DAPART (Diagnostics After PARTicles) is necessary because another type of information, their relative locations in $\mathrm{R}, \mathrm{Z}$ space, can be obtained only by plotting information from individual particles. These plots are expensive, because they require a separate pass through the buffering routines, but are essential to the formation of certain types of insight. One could find a way to put all this information on one frame and thus do these plots in DPART but that's too much information on one frame even for me! 


\section{VI.C) DASPECS Plots Macroscopic Source Terms from Each Species}

The other way to get particle source information is to sum over particles of each species to get the macroscopic moments, density DEN, temperature TEM and drift velocities $U$. TRANS orchestrates these activities as the particles go through PARMOV; these quantities are necessary for the field solution. We plot this information either in contour plots CPLOTs, vector plots VPLOTs, of averages in one or the other direction with APLOTs.

\section{VI.D) DSOURSE Plots Species-Summed Macroscopic Source Terms}

Another of the many activities of TRANS is that when it is finished, when control is returned to main, it will have summed the various species together to form the total charge and current density for use in the field solution. The diagnostic entry DSOURCE is the place where these sources are plotted in various ways.

\section{VI.E) DFIELD Plots Potentials and Fields $\mathrm{A}, \Phi, \mathrm{E}$, and $\mathrm{B}$}

By this point, we have nothing to say that will be a surprise. After the fields are calculated, we plot various parts and pieces with the usual complement of CPLOTs, VPLOTs and APLOTs. It is worth noting that we plot the electron information here because it is not known until after the fields have been advanced in time.

\section{VI.F) HISTORY Plots Histories After "Unraveling" Pointer}

The difficulty with maintaining historical information for plotting at the end of the run is obvious; it uses too much storage. Another approach is to maintain smaller arrays for each of the quantities for which history plot are needed, retain only the most recent entries, and plot before we have obtained more new entries than we can store. We have implemented this strategy in GYMNOS. We dimension the history arrays ITHMX and. therefore, save the last ITHMX values: We plot these values the next time HISTORY is called. To achieve full coverage over the course of a long run, one needs to call HISTORY (controlled by IHIST) more frequently than every ITHMX time steps.

To avoid constantly working with a "pushdown" stack when each new entry is added. we keep track of a pointer that contains the location of the oldest element in the file. Every IHIST time steps, a new element is stored there and the pointer is moved to the next oldest entry. When HISTORY is called the array is reordered before plotting so that the oldest information is in the first cell and the latest information is in the ITHMX cell, or the IT cell if IT < ITHMX. Provisions are made to save plot the lastest ITHMX values even if IHIST is bigger than ITHMX, thus causing the loss of some information.

The coding that accomplishes this is illustrated by an example of the history of the electric field energy EEF. For convenience the storage is kept in the common block HISTY that is spread throughout the code using the cliche SHIST.

\footnotetext{
common /histy/ mn6f, ihistpt, eef $(0: i \operatorname{thmx}), \ldots, \operatorname{mn} 61$

c TRANS Historical data saved every timme step in TRANS.

$c \quad$ IHISTPT is the pointer to the oldest data.
} 


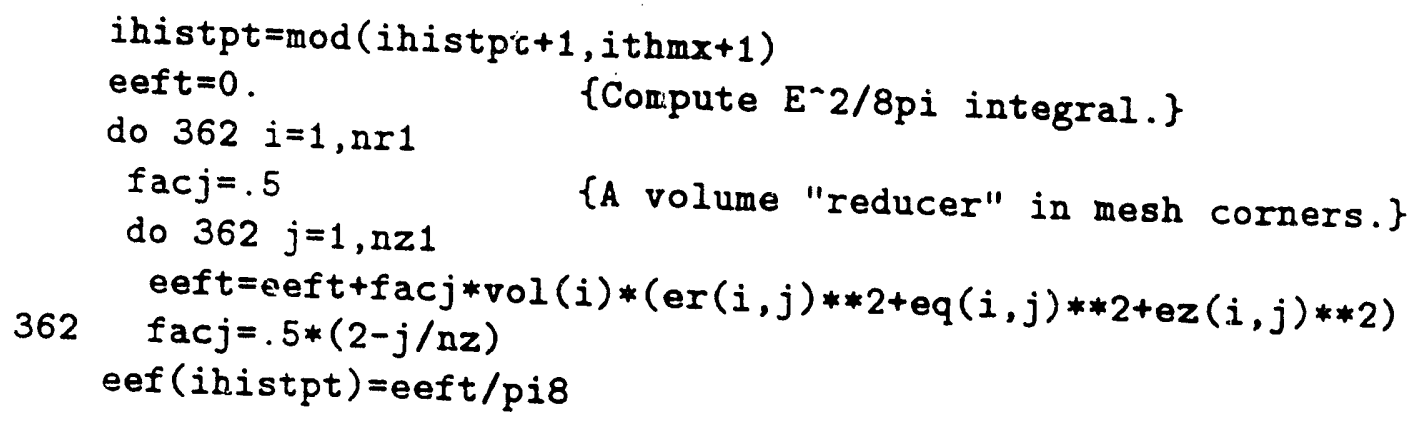
The following Fortran in the subroutine DIAGNOS unrolls and plots the latest ITHMX
history values of EEF.

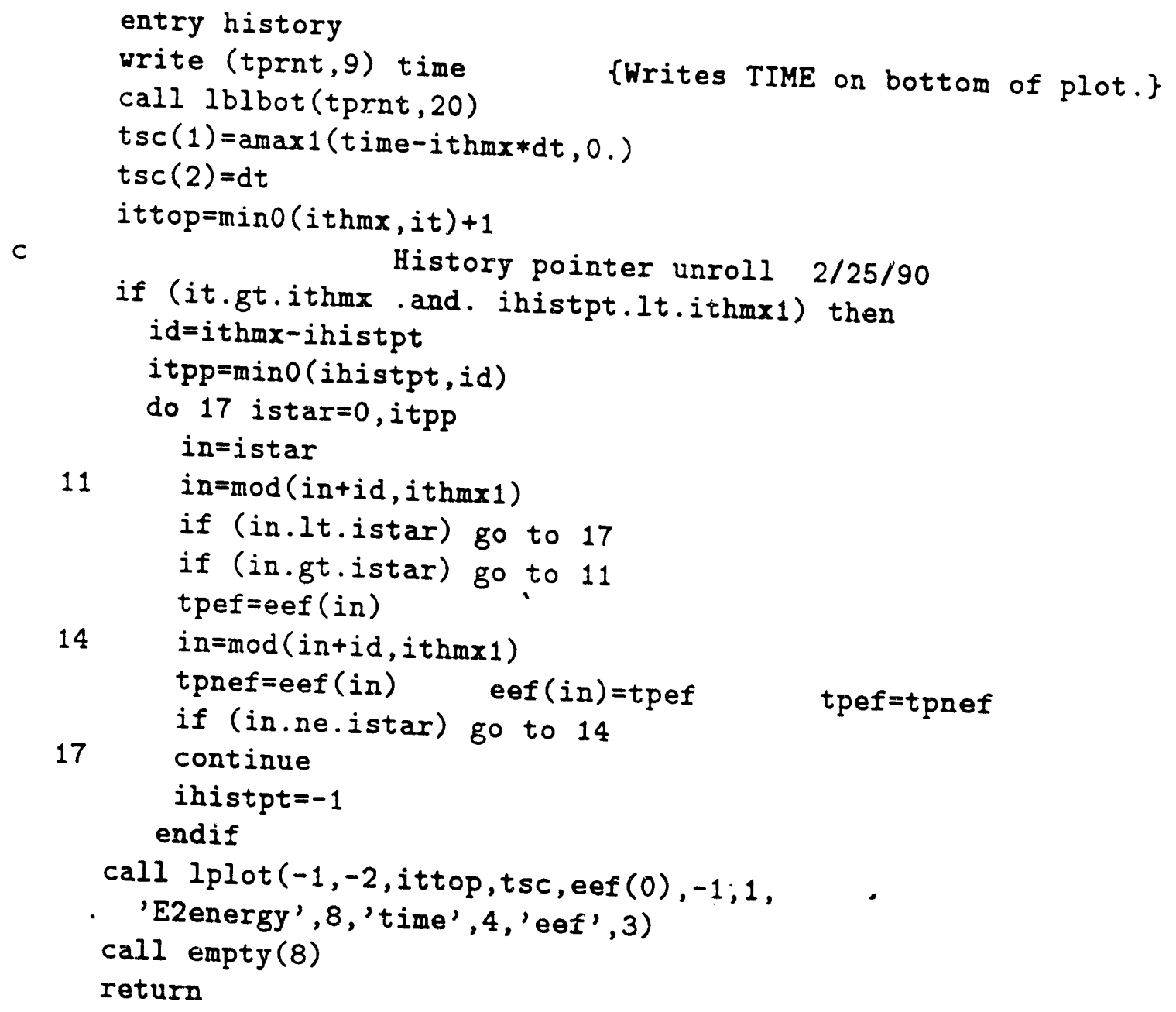




\section{VII) Running GYMNOS (CTSS)}

We have set up the file GYMNOS to contain some sample input decks as well as the main code. Thus when GYMNOS is run as input to the COSMOS controller, the input files and controllee are generated whin COSMOS is finished.

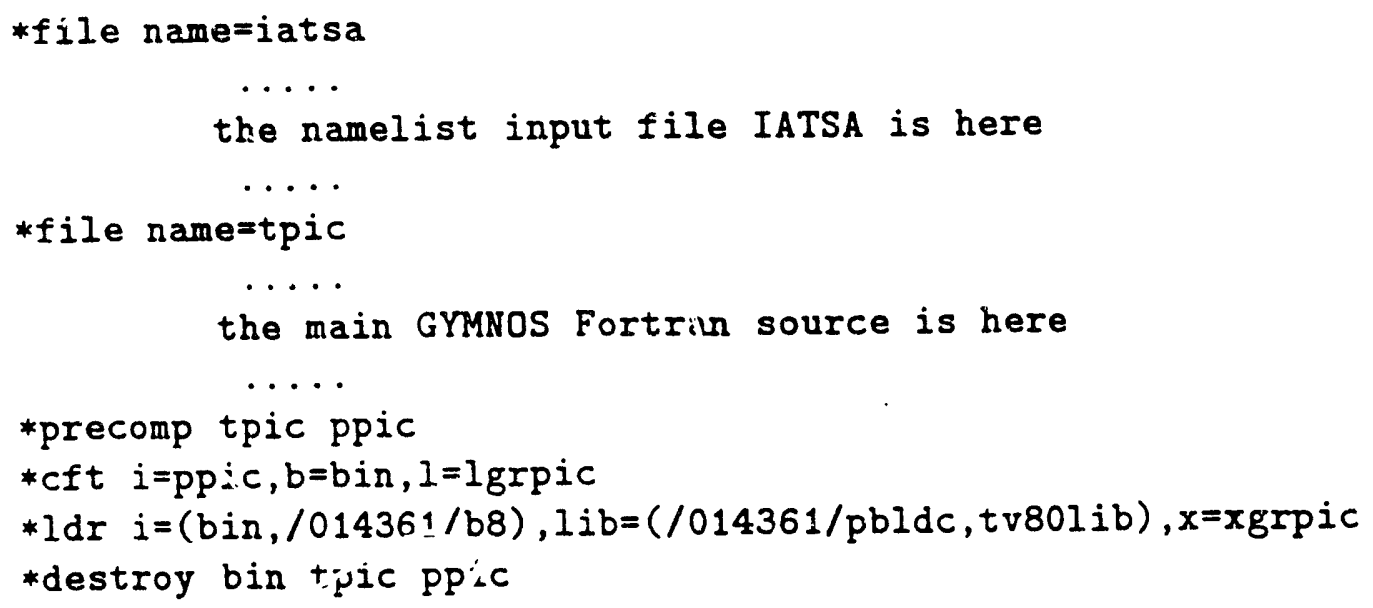

Here the controllee is XGRPIC and the ATSA run can be started by typing XGRPIC AT.iA / 13.

XGRPIC is a creation by the loader LDR of the fortran inary BIN created here from the GYMNOS source here called PPIC. The loader will merge BIN with the other binary file B8 containing the support routines such as VDKR, VTRIK, etc. (compiled earlier and residing in local file spice) and the plot library PBLDC.

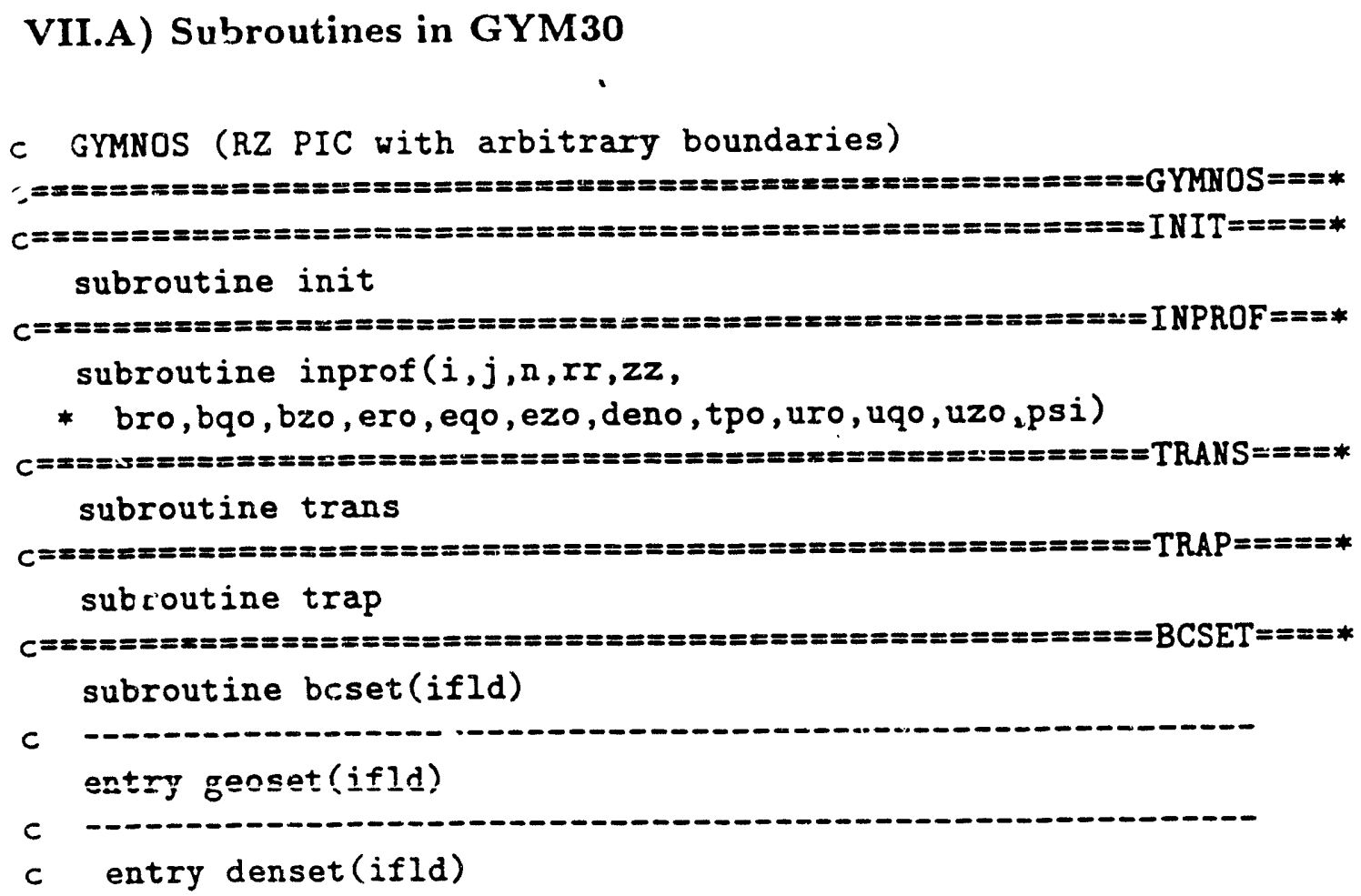




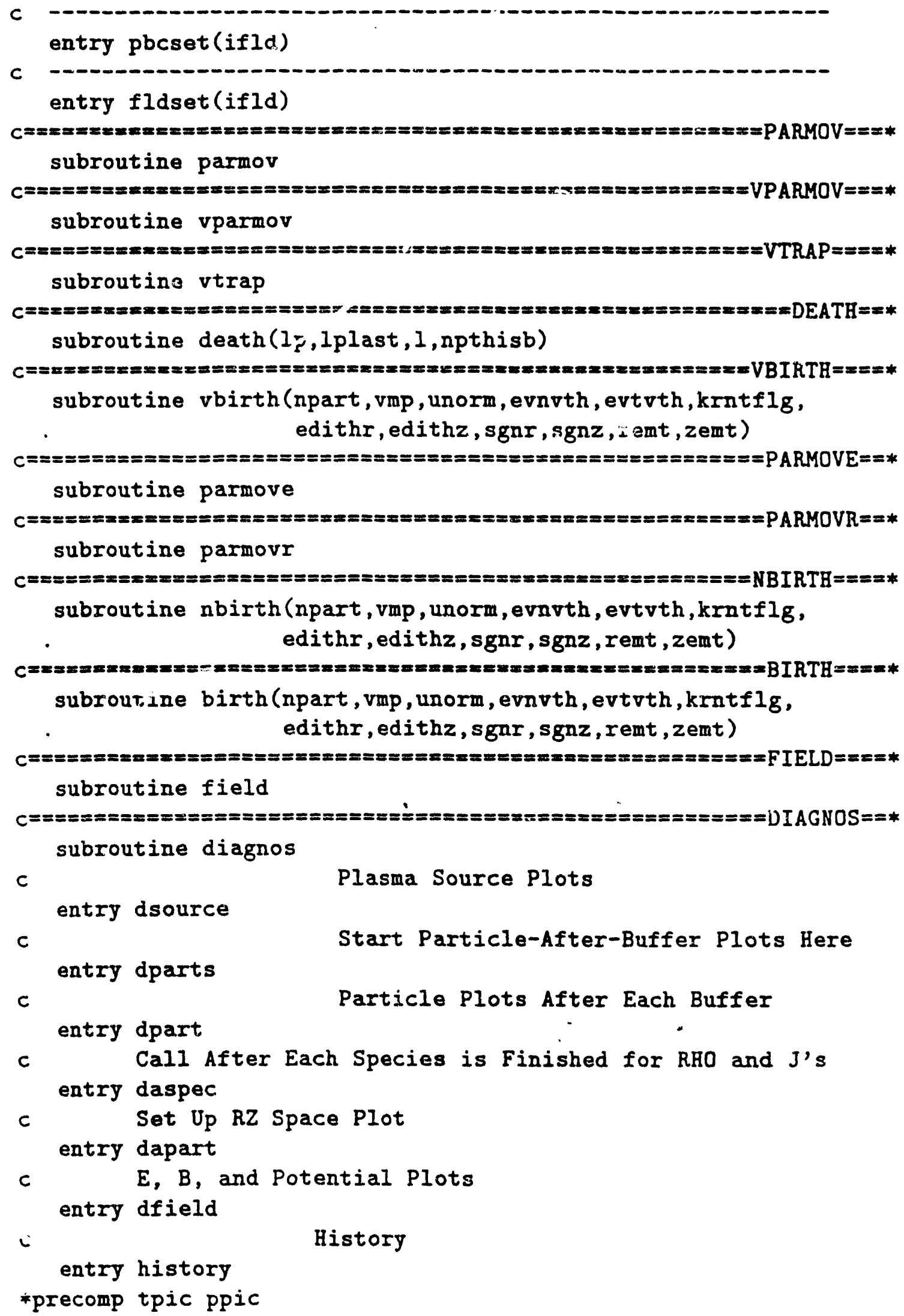


*cft $i=p p i c, b=b i n, 1=1$ grpic

*ldr $i=(b i n, / 014361 / b 8), l i b=(/ 014361 / p b l d c, t v 801 i b), x=x g r p i c 7$

*destroy tpic ppic

\section{VII.B) Subroutines in GYMS22}

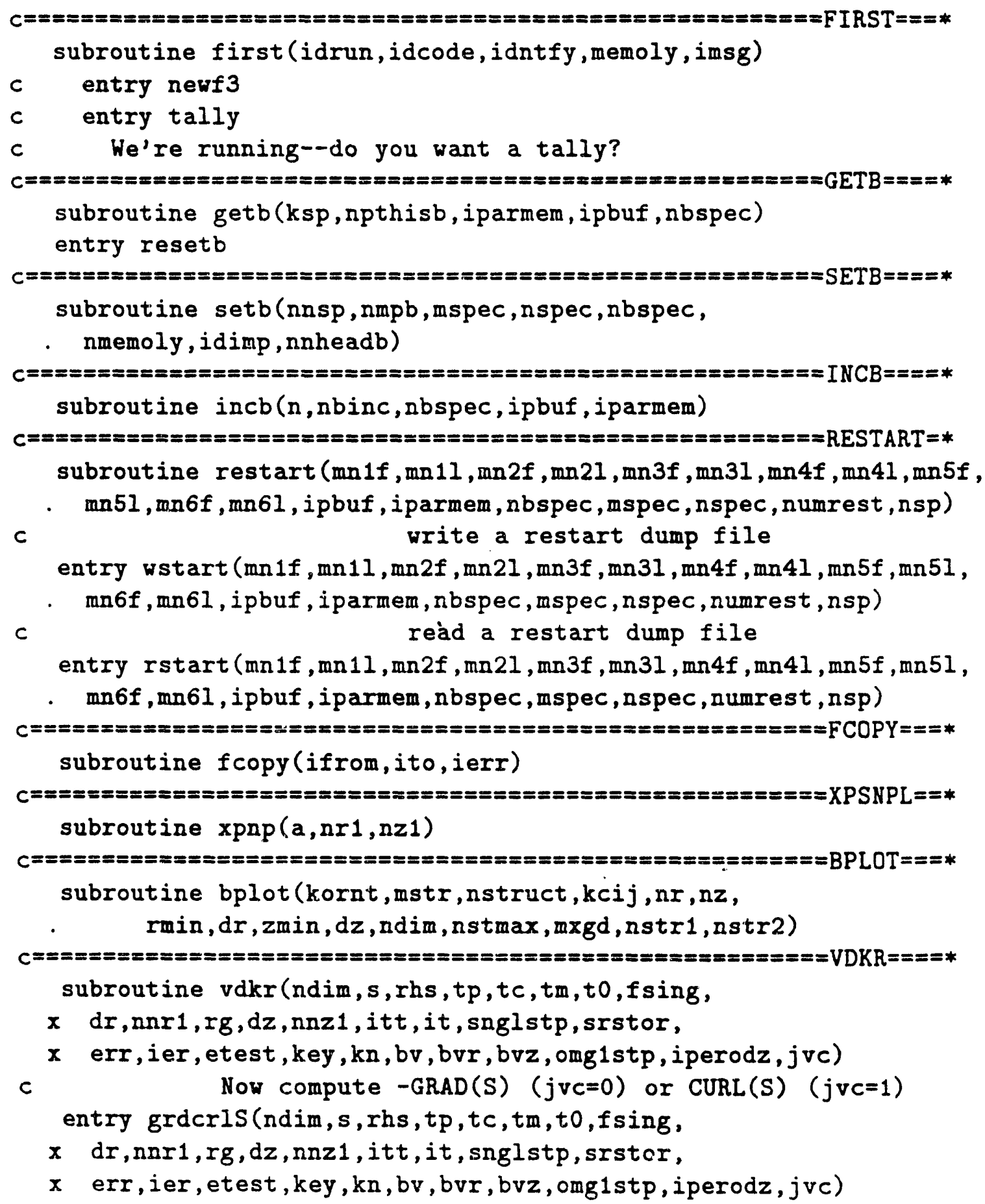




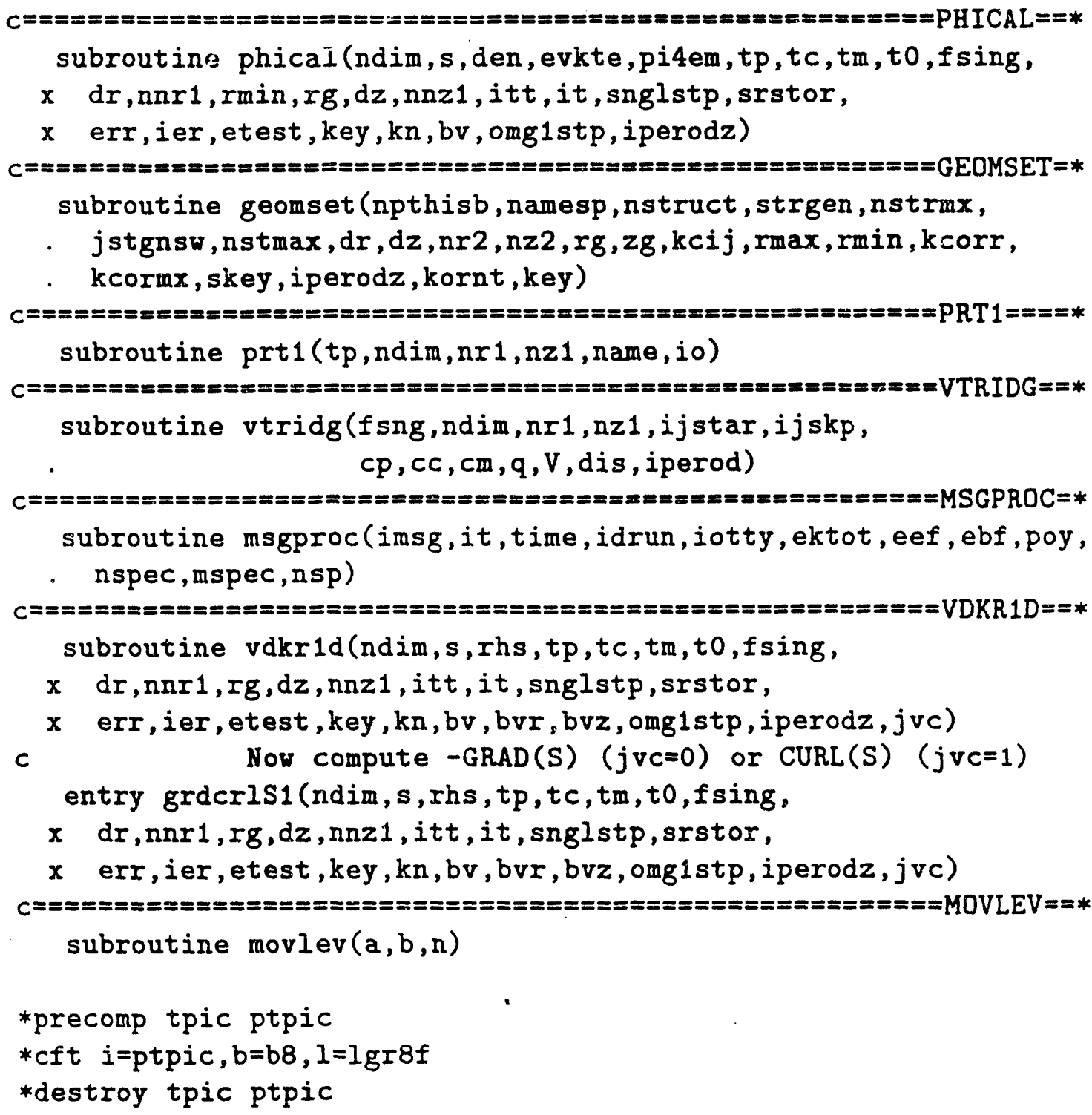

\section{VII.C) A Test Case: The LBL Source}

We give here a typical input deck for an LBL source simulation. The first line is an ASCCI label that is printed across the top of all plot frames. Next is the namelist input for namelist NDATA, followed by namelist GSET, and finally, input for namelist NECHSP once for each of the two species.

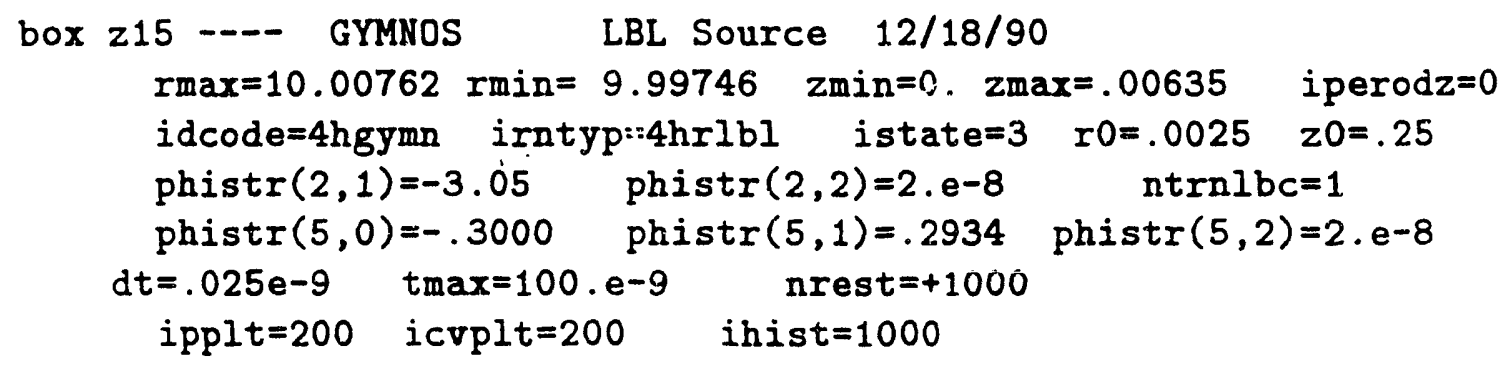


$\$$

nspchk $=2 \quad$ jstgnsw $=-1$

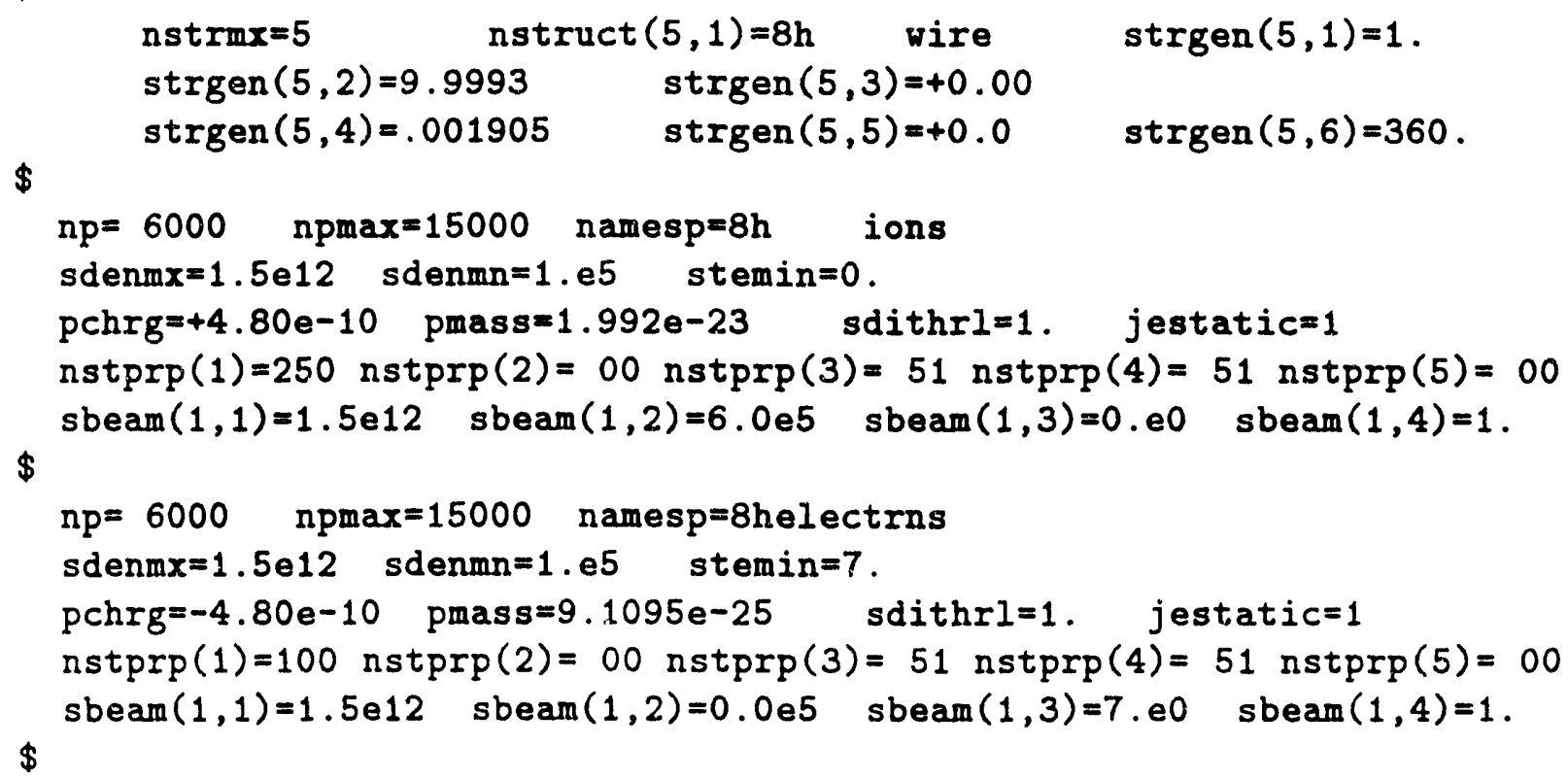




\section{VIII) Summary and Conclusions}

Most of the information in this document is in fact contained in the comments statments within the code. The FORTRAN in the code remains the ultimate reference. Plans for the immediate future certainly include the proof testing of the VPARMOV series and the implementation a fluid pusher extracted from a code written in DWH's previous life so that selected species (perhaps even neutral gases) can be represented by a fluid.

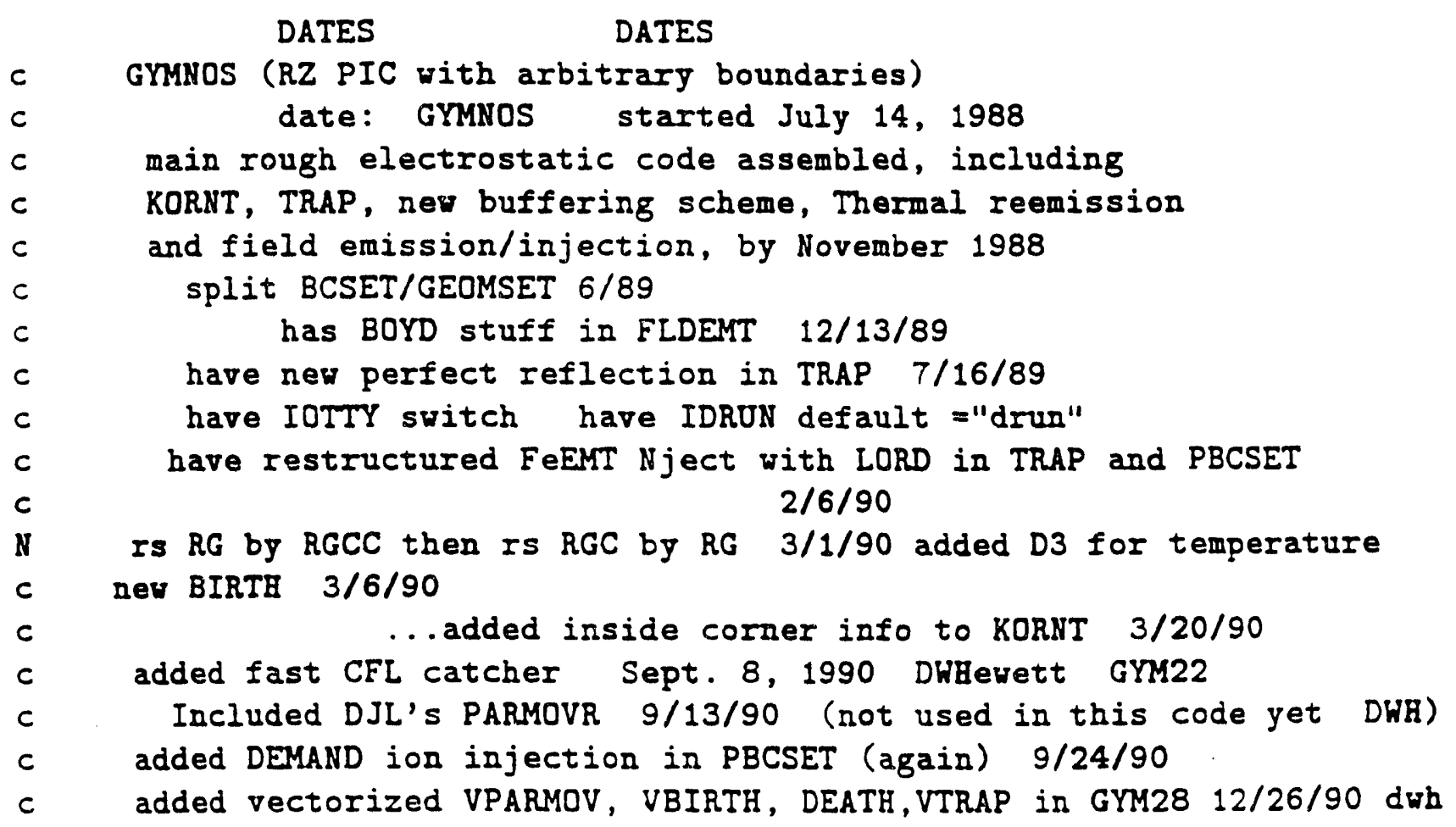




\section{APPENDICES}

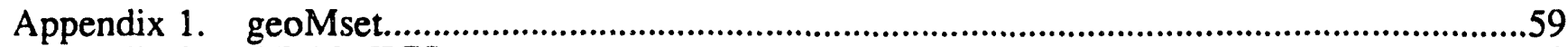

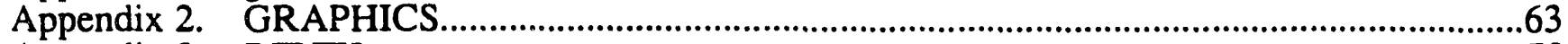

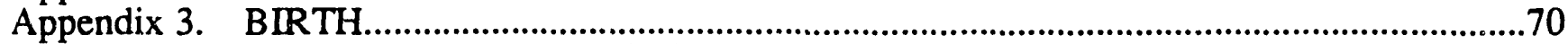

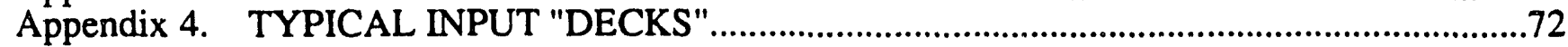

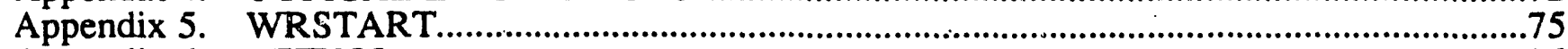

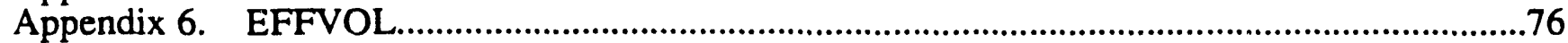

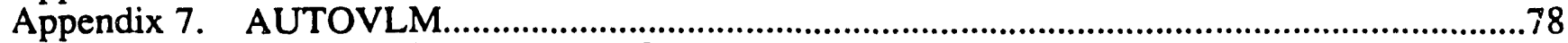

Appendix 8. PARTICLE BUFFERING ROUTINES: SETB, GETB, RESETB........................79

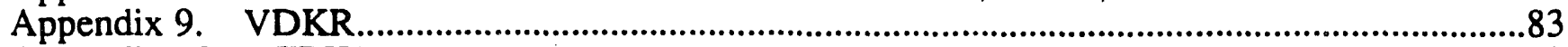

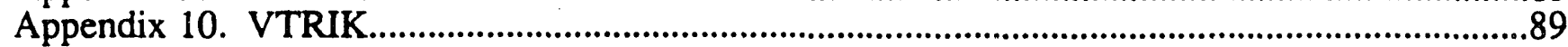

Appendix 11. VPARMOV/VTRAP/VBIRTH/DEATH........................................................90

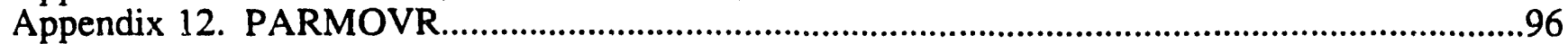

Appendix 13. Santa Fe Numerical Simulation Abstract.........................................................98

Appendix 14. GYM28 SUMMARY SHEETS....................................................................... 102 
Appendix 1. geoMset

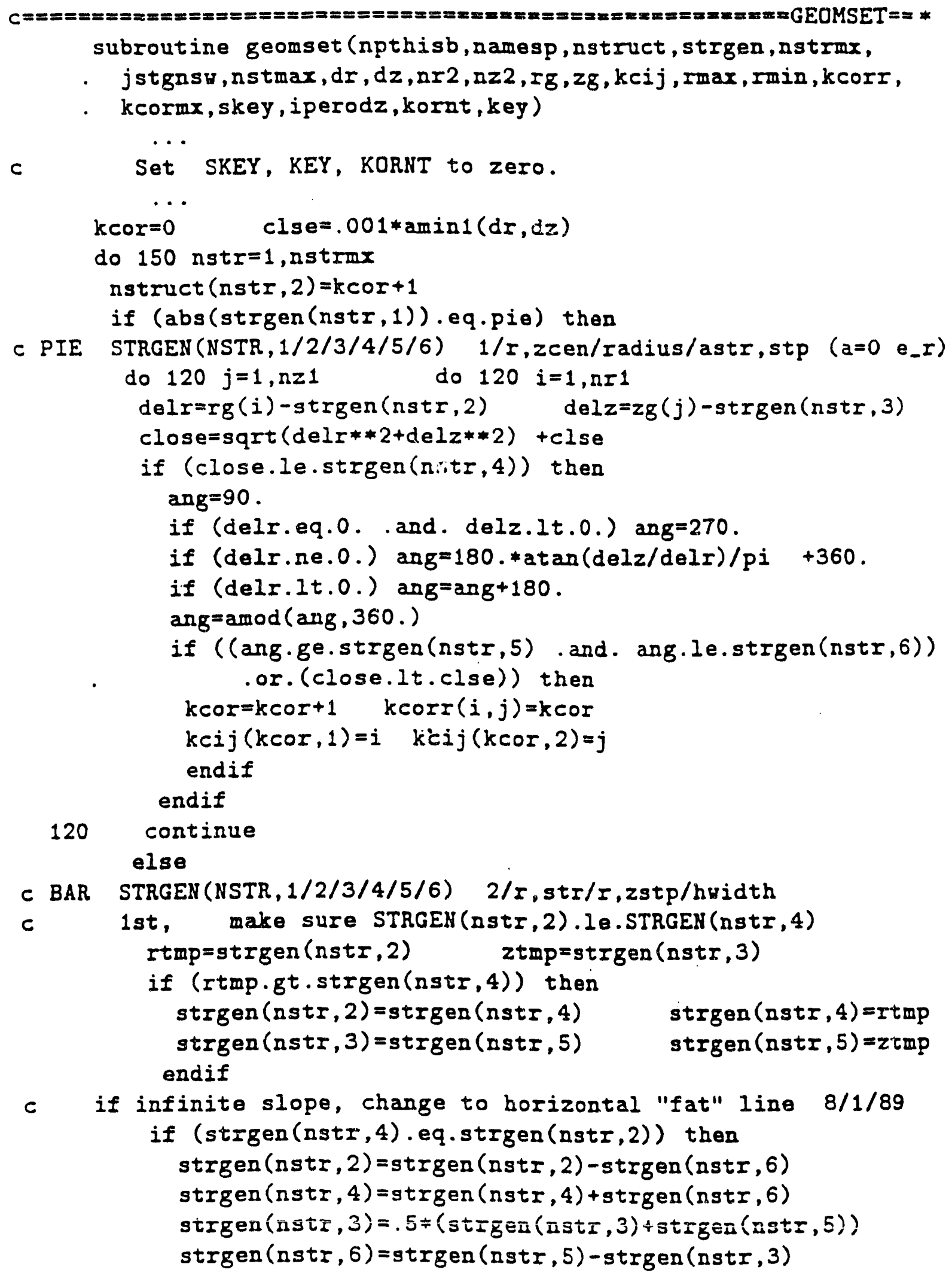




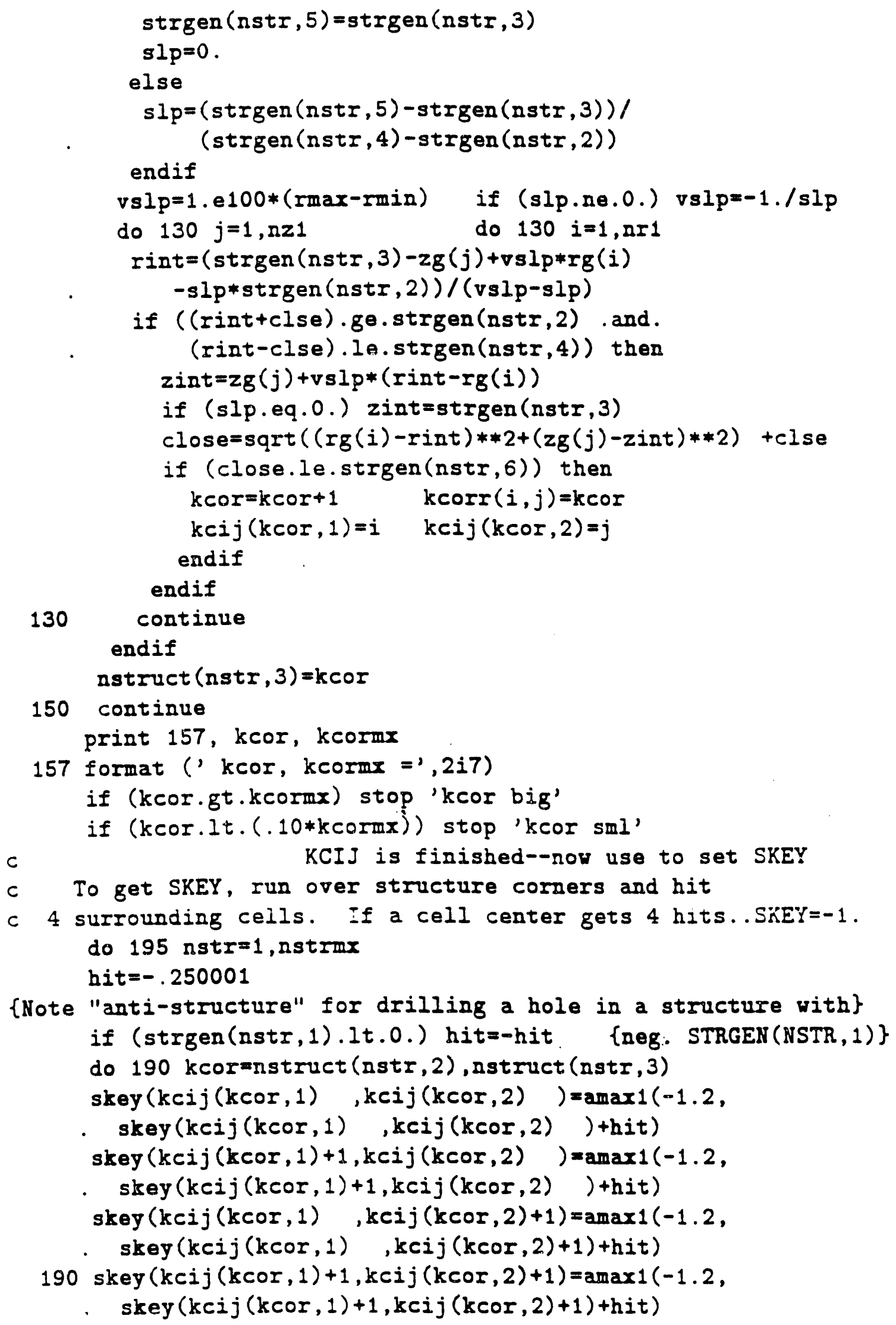




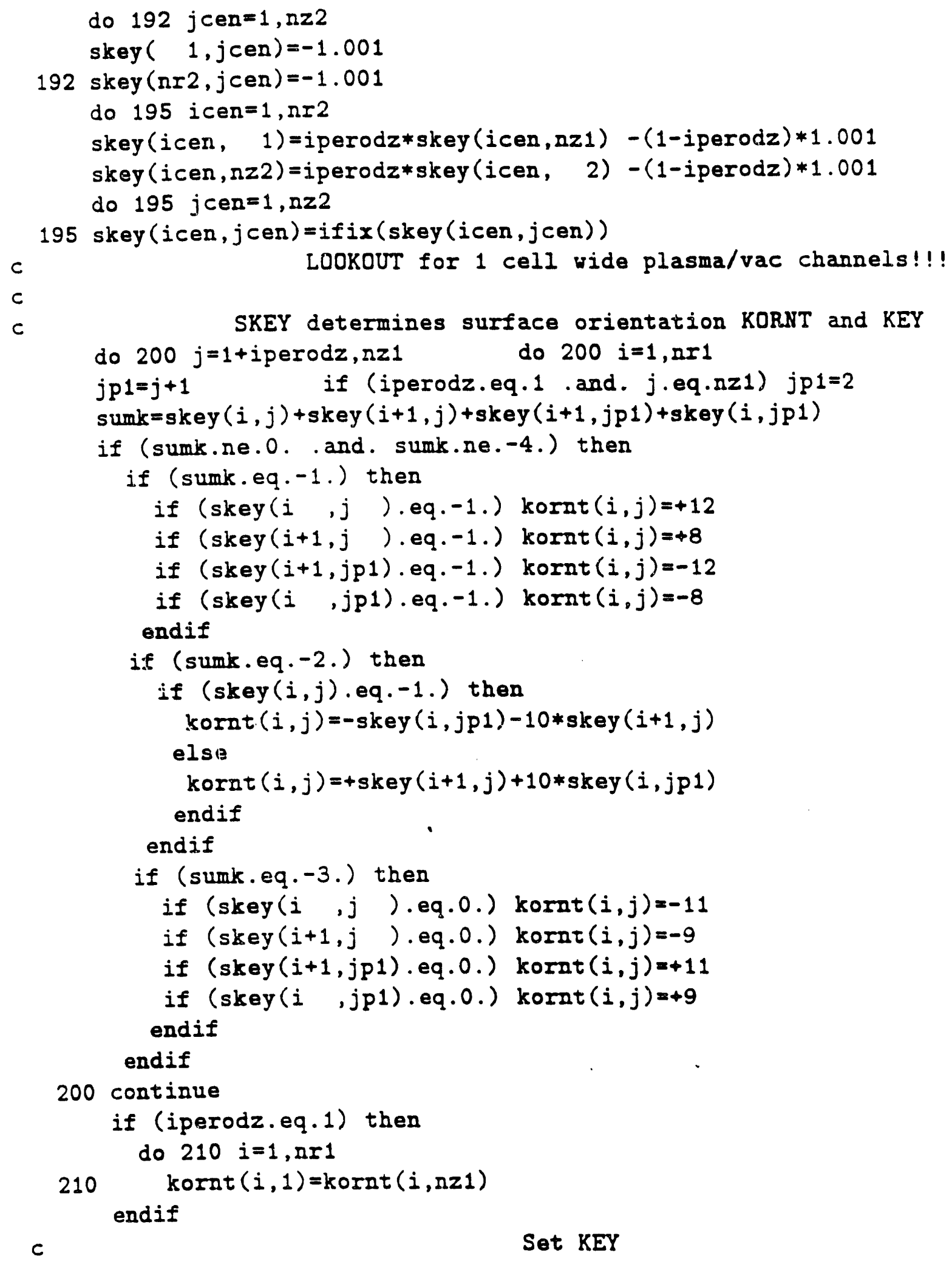




$$
\begin{array}{lc}
i c=\min 0(i, n r 1) & j c=m i n o(j, n z 1) \\
\operatorname{key}(i c, j c)=-1 & \operatorname{key}(i c m 1, j c)=-1 \\
\operatorname{key}(i c, j c m 1)=-1 & \operatorname{key}(i c m 1, j c m 1)=-1 \\
\text { endif } &
\end{array}
$$

255 continue

return

end 


\section{Appendix 2. Graphics}

Almost all piot libraries have the basic routines needed: routines that draw and scale axes. line plotters (with linear-linear. log-linear, etc.) contour plotters, vector plotters and point plotters. GYMNOS uses a personal plot package maintained by DWH but a similar one (derived from this one in 1983 and extended by LANL CTR Division) is available. Our version itself evolved from an even earlier version written by many people in LANL in the early and middle 70's. Our bias (and therefore our advise) is to get by with minimal embellishments such as fancy labels. These codes use lots of CPU time and we wants LOTS of pictures to build intuition. Only one in 10,000 pictures ever gets published but they all rob CPU cycles from the computational effort. Therefore, every effort is made to display as much information as cheaply and crudely as possible. Extra effort is expended only on those graphs intended for display. The plot package is capable of many things but, not surprisingly, certainly does not make publication quality pictures! Here is enough documentation to use the plot routines in GYMNOS.

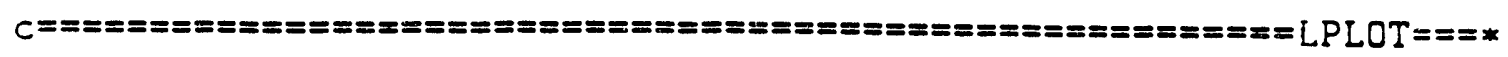

subroutine lplot (imx, imy, npts, $x, y, i n c$, iop,

1 Ititle, ntitle, Ixname, nxname, lyname, nyname)

LPLOT plots NPTS values of $x$ and $y$ and connects them with a line. It draws a box around the plot with scaling and places labels on the left and bottom axis.

The plot's position on the frame is determined by IMX, IMY. An IMY=1 specifies that the $y$-coordinate range spans a full frame; IMY $=2,3$ specify the upper, lower halves of the frame respectively; and $I M Y=4,5,6$ specify the upper,middle, lower thirds of the frame. IMX=1 specifies that the $x$-coordinate range spans a full frame, \& $I M X=2,3$ specify the left, right halves of the frame. IMX=iais (4), IMY $=1$ gives a square plot area--useful if the $X$ range and $Y$ range are equal to see proper aspect ratio. IMX $=5,6$ are not allowed. For example, (imx,imy) $=(1,1$ ) specifies a plot filling the full frame, and $(3,3)$ specifies a plot in the lower right-hand quadrant. Frame advance is automatic with the first plot that extends into the upper left-hand corner of the frame. Such a plot must be the first in any plot sequence intended to appear on one frame. The plot's range usually is expanded to a "round" decimal number by the automatic scaling routnes from the minimum range implied by the data. Expansion may be prevented by appending a minus sign to either/both IMX and IMY in any plot call. For example, if $x m i n=0.17$, xmax $=359.78$, and IMX is positive for automatic scaling, the $x$-axis scale goes from 0.0 to 400.0 . If IMX is negative for exact scaling, the $x$-axis scale goes from 0.15 to 359.78 with "rounded" tick marks at the correct positions. 
Summary of IMX,IMY positions

$$
1,4
$$

$$
1,2
$$

1,1

$$
1,5
$$

1,6

$$
\begin{array}{llll}
2,2 & 3,2 & 2,4 & 3,4 \\
2,3 & 3,3 & 2,5 & 3,5 \\
& & 2,6 & 3,6
\end{array}
$$

$(4,1) \quad 1,3$

(square)

4,1 gives square full frame

IMX, IMY < 0 gives exact scaling; > 0 gives automatic scaling.

|NPTS| The number of $y$ values to be plotted. NPTS $<0$ draws a curve onto a frame previously setup by LPLOT with IOP<O.

$X(Y)$ is the table of abcissa(ordinate) values to be plotted.

|INC| The spaciong between successive $X$ and $Y$ elements to be plotted. INC<O $X(1)=X$ min and $X(2)=D X$

eg:---If we want to plot 20 points separated by 3 locations with $x(1)=X m i n$ and $x(2)=D X$, we chose NPTS $=20$, INC $=-3$ so that the code will search through 60 locations in order to find the 20 elements to plot.

IOP $>0$ scales and draws frame and plots data from $X$ and $Y$.

$<0$ draws frame and scales but does not drav curve.

$=1000 * \mathrm{~N} 3+10 * \mathrm{~N} 2+\mathrm{N} 1$.

$\begin{array}{ll}\mathrm{N} 1=1 & \text { Iinear } X-a x i s \text { and linear } Y \text {-axis } \\ N 1=2,3,4 & \text { linear-log, log-linear, log-log } \\ N 2 & \text { Indicates the decimal equivalent of the character }\end{array}$ to plotted at the data point. Default is no symbol.

N3 $=0$ The points are not connected by lines.

$\begin{array}{ll}0<N 3<9 & \text { A symbol is placed at the first and } \\ \text { Other points are connected by lines. }\end{array}$

LIITLE is the the title for the graph.

LX(Y)NAME is the the label for the $X(Y)$-axis.

NTIILE, NX(Y)NAME is the number of characters

in ltitle,LX(Y)NAME, maximum of 16 .

NYNAME < 0 scales and tick marks for plot not drawn. 


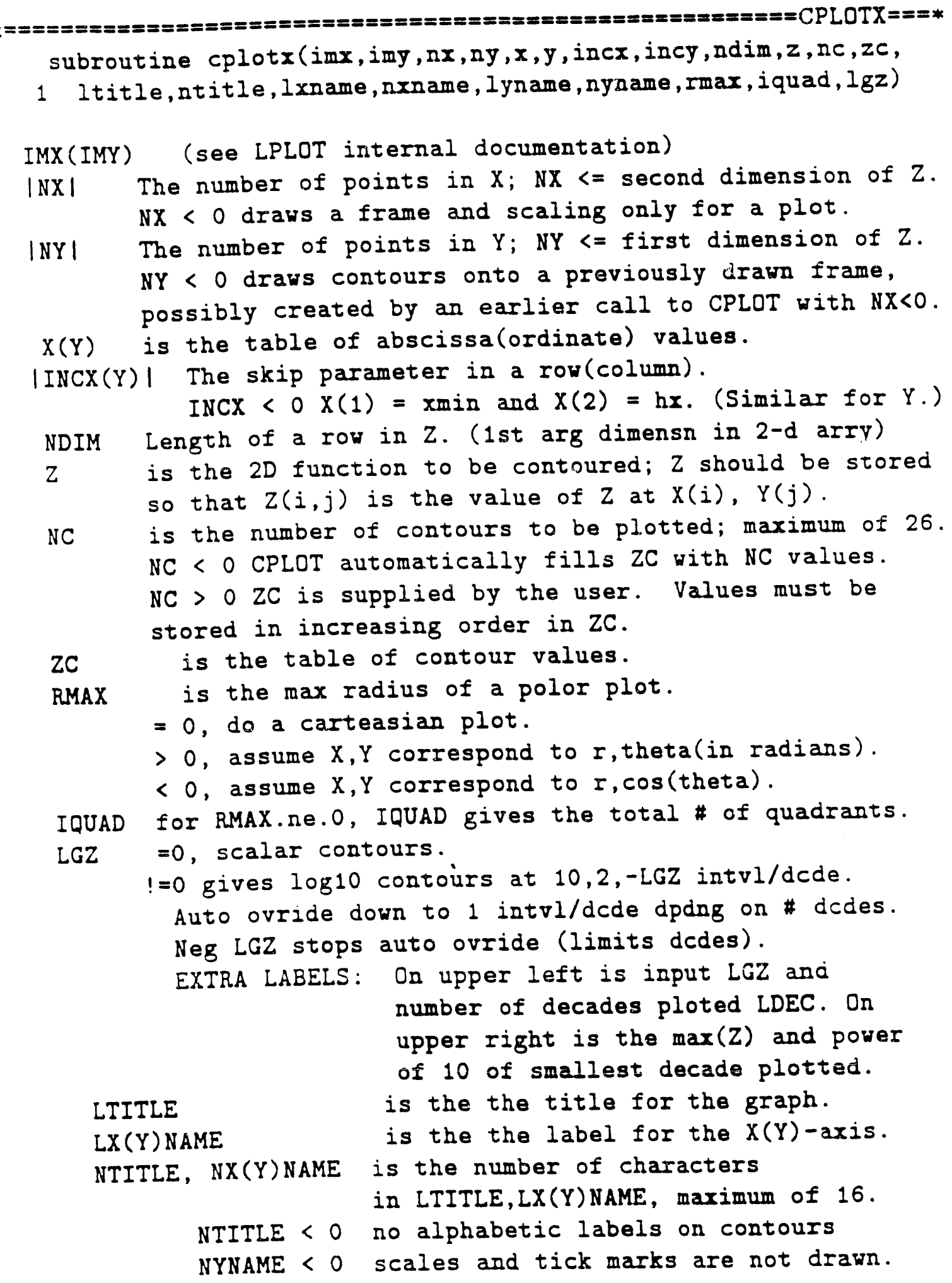

LTITLE

LX(Y)NAME NTITLE, NX(Y)NAME

NTITLE < 0 no alphabetic labels on contours NYNAME < 0 scales and tick marks are not drawn. 


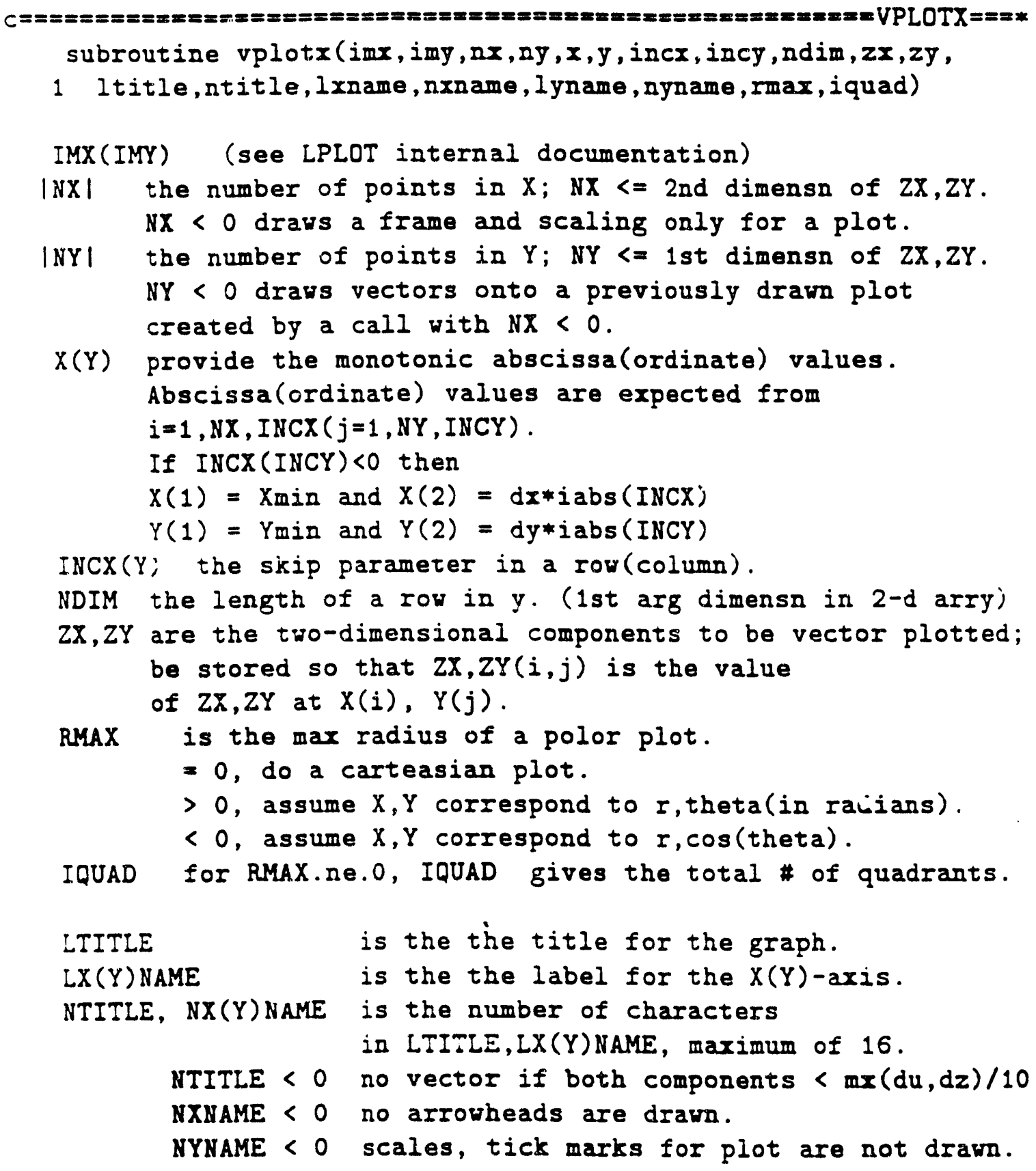

LTITLE

LX(Y) NAME

NTITLE, NX(Y)NAME

NTITLE < 0 no vector if both components $<m x(d u, d z) / 10$ NXNAME < 0 no arrowheads are drawn.

NYNAME < 0 scales, tick marks for plot are not drawn. 


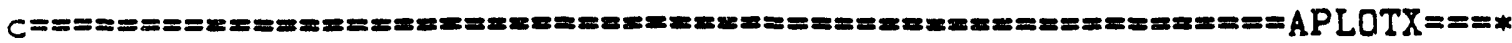
subroutine aplotx(s,ndim,avy,avx,xsc, ysc,mxya,myya,mxxa,myxa, $1 n x, n y, n f t 1, n f t 2,1 t i t l y, n t i t l y, 1 t i t l x, n t i t l x, i x a, i y a, i r z)$

APLOTX( $Y$ ) averages over a matrix of values in one direction and plots the results with respect the other direction. This subroutnes only performs the averaging calculation; it calls LPLOT to drav the resulting curve.

$S$ is the $2 D$ table of values 1 st dimensioned NDIM.

AVX (AVY) is array that holds the average of $S$ in the $x(y)$-direction. It is dimensioned $N X(Y)$ in user's program. If a user wants only average returned from the APLOT, set IXA(IYA) $=0$. $X S C(Y S C)$ is the $x(y)$-axis information. (i.e. XSC $(1)=X m i n, X S C(2)=D X)$

MXXA , MYXA , MXYA , MYYA

indicates the location and length of the $x(y)$-axis. the meaning is the same as in LPLOT.

(i.e. MXXA,MYXA = IMX,IMY for the xavg plot) (see LPLOT $i \cdot$ ternal documentation)

$<0$ gives exact scaling; > 0 gives automatic scaling.

$N Y(X)$ is the (usual) number of points in the $y(x)$ average.

NFT1 and NFT2 specifies a band of points to average over--X-direction only.

NFT1 is $i$ index of the 1 st cell in the average calculation. The value for averaging over the whole function is 1 .

NFT2 is \# of cells in the band to average over. The value for averaging all ceil in the $x$-direction is $N X$.

$N X(Y)<0$ Vertical scale fixed by previous call to LPLOT.

LIIILX $(Y)$ title for the average plot in the $x(y)$-direction.

$\operatorname{NTITLX}(Y)$ \# of characters in LTITLX(Y); max of 16.

$\operatorname{IXA}(Y)=1$, average plot in $x(y)$-direction, drawn by LPLOT.

$=0$, average made not plotted, returned in AVX $(Y)$.

$=-1$, neither an average nor a plot is. made.

IRZ $=0(1)$ label graphs $x(r)$-avg and $y(z)-a v g$. 


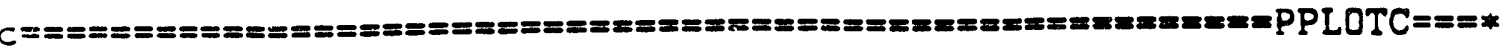

subroutine pplotc(imx,imy,npts, $x, y, i n c, z, z \min , z \max )$

PPLOT plots values in $X$ and $Y$. Each point is ploted as dot, adjacent points are not connected. PPLOTC plots the point only if its $Z$ < ZMAX and > ZMIN. Both subroutnes assume that the frame, scale and labels for this IMX and IMY plot have been generated by LPLOT with IOP $=-1$. Only linear-linear scaling is allowed. NPTS is the number of elements in $X, Y$, and $Z$.

IMX (IMY) (see LPLOT internal documentation)

IMX, IMY < 0 gives exact scaling; > 0 gives automatic.

NPTS is the number of elements in the arrays $X, Y$, and $Z$.

$X(Y)$ is the table of abcissa(ordinate) values.

IINC| The spacing between successive $X$ and $Y$ elements.

INC $<0 X(1)=X \min$ and $X(2)=D X$

$Z$ is a function of $X$ and $Y$.

$\operatorname{ZMIN}(Z M A X)$ is the smallest(largest) value plotted.

Typically, a user encodes the TIME his code has reached and uses LBLBOT to write it at the bottom of the frame.

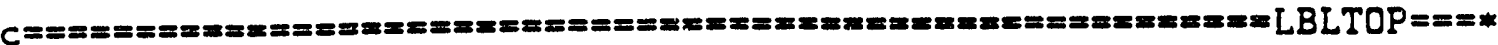

subroutine lbltop(label,nlabel)

LBLTOP enables a user to specify an 80-character label at the top of a page of plots. LBLBOT enables a user to specify a 40-character label at the bottom of a page of plots. Labels are centered at the top and bottoin of the page. Subroutnes should be called before any plot calls on a page. Label calls remain in effect for each succeeding page until another label call with a different character string is given. e.g.

call lbltop ('plasma physics plotting package', 31)

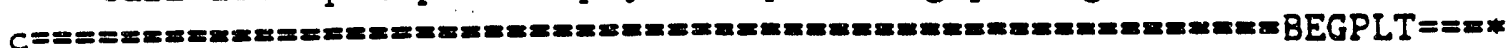

subroutine begplt (iname,ntitle,ltitle)

BEGPLI initializes the plotting routnes; must be called before any plotting is done. Creates a file named f3abcdox in the user's local file space on CTSS. The $a, b, c$, and $d$ in the name are 4 characters specified by user in INAME. e.g. If INAME='abcd', graphics output is witten into $f 3 a b c d 0 x$. If additional file space needed, new graphics file is created by incrementing the 4 th letter as followg: $f 3 a b c e 0 x$, $f 3 a b c f 0 x$, etc. For unique eye-readable microfiche title, call the fortlib routine, SETBOX, before BEGPLT.

e.g. call setbox('box 130 reversed field pincb 1', 30) The string must start with your box number and the following 23 


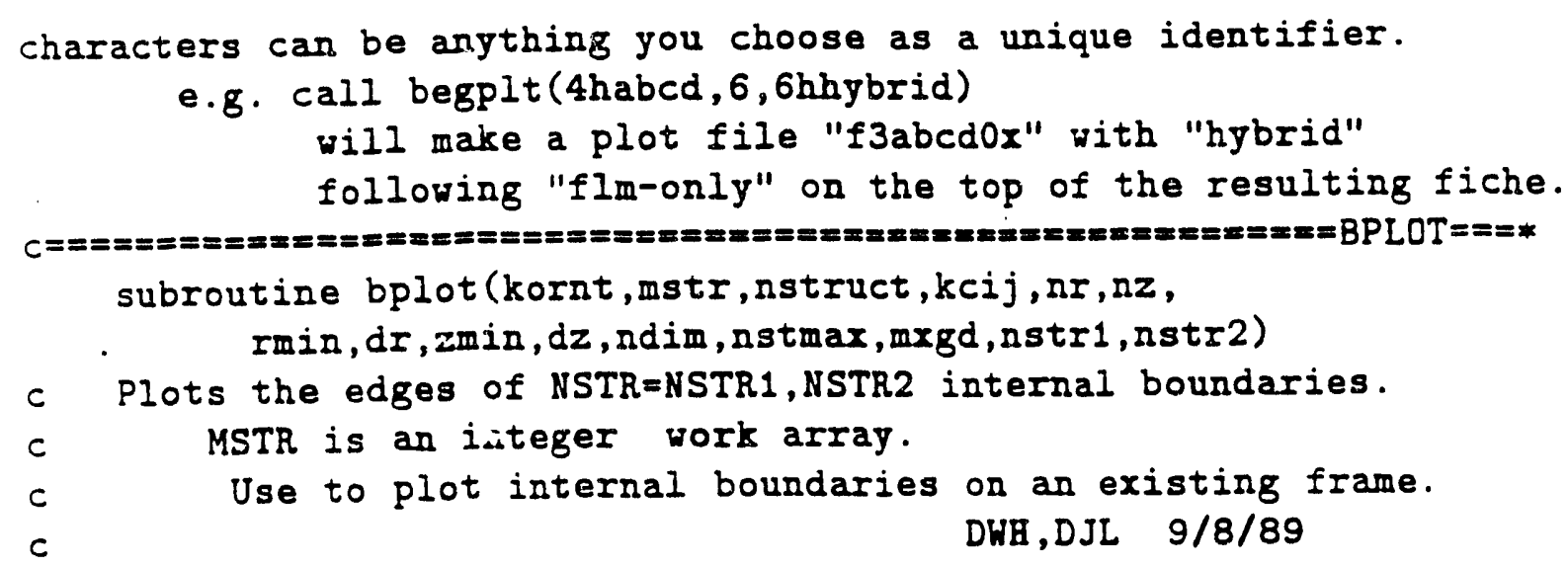




\section{Appendix 3. BIRTH}

BIRTH is the routine that creates new particles during the course of a run. It is called only from routine TRAP during the processing of buffers by PARMOV as directed by TRANS. BIRTH creates NPART particles just before they experience the first order velocity extrapolation and then are integrated or "accumulated" into the source terms of their species $\mathrm{N}$.

The new particles are created at location REMT.ZEMT with a thermal spread given by VMP. the most probable thermal velocity. BIRTH orients the normal velocity magnitude U.NORM using the values COSA.SINA (called SGNR,SGNZ in TRAP) when called by the emit/inject section and using KORNT values for the orientation of the emitting corner if the thermal reemission section is the calling routine.

call birth (npart, vmp, unorm, evnvth, evtvth, kornt (icor,jcor), edithr, edithz,sgnr,sgnz, Ig(icor), zg(jcori)

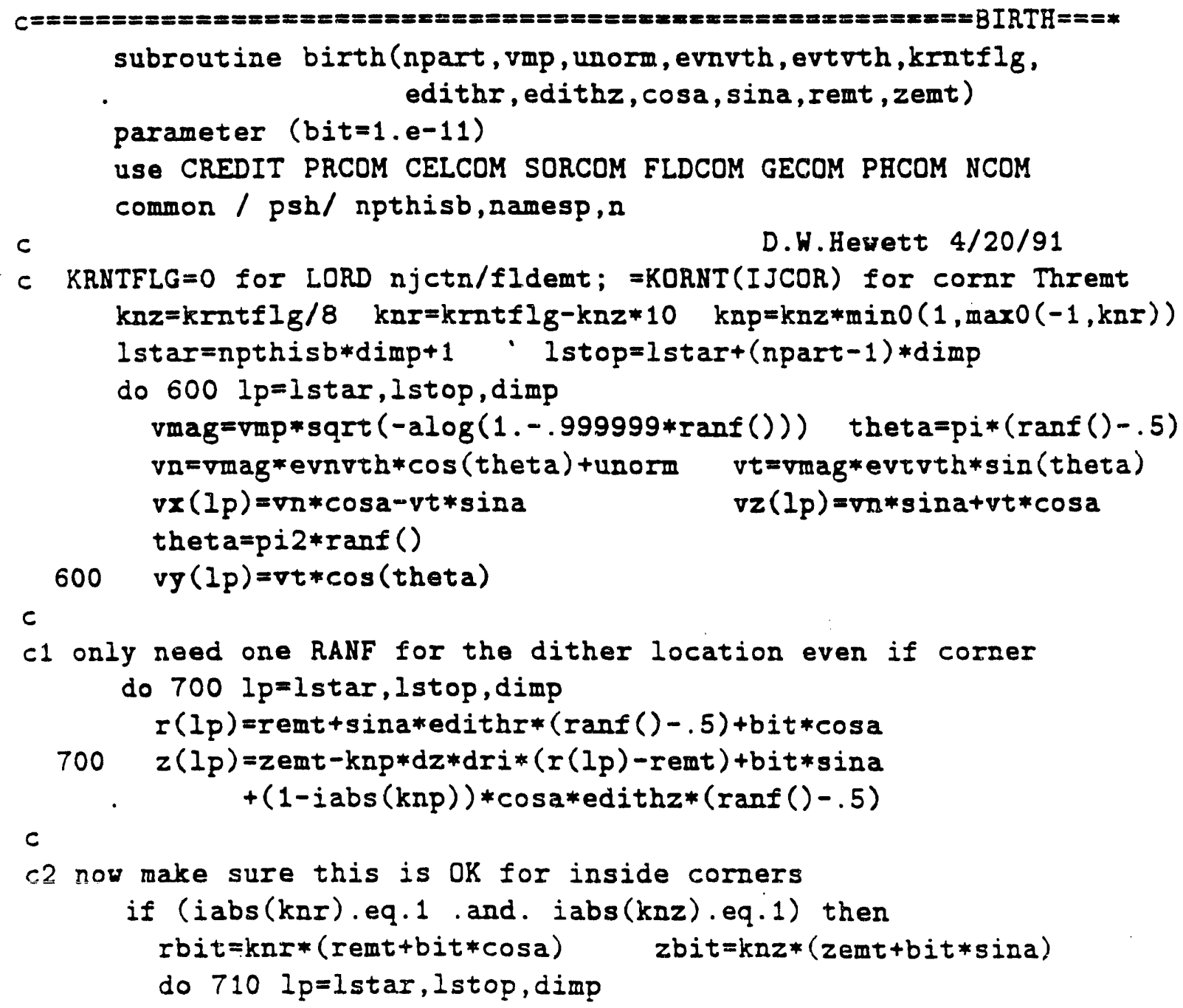




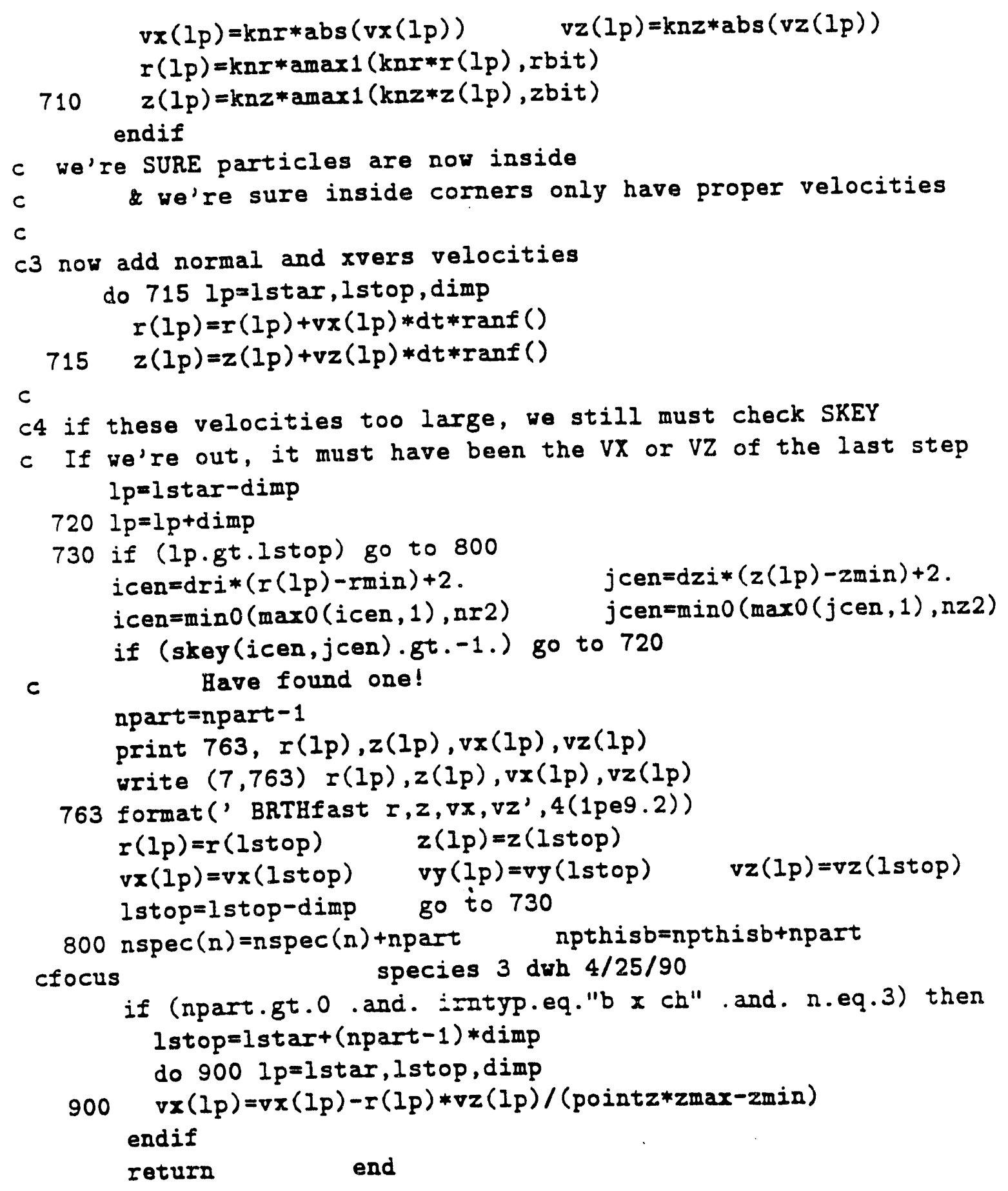




\section{Appendix 4. Typical Input "Decks"}

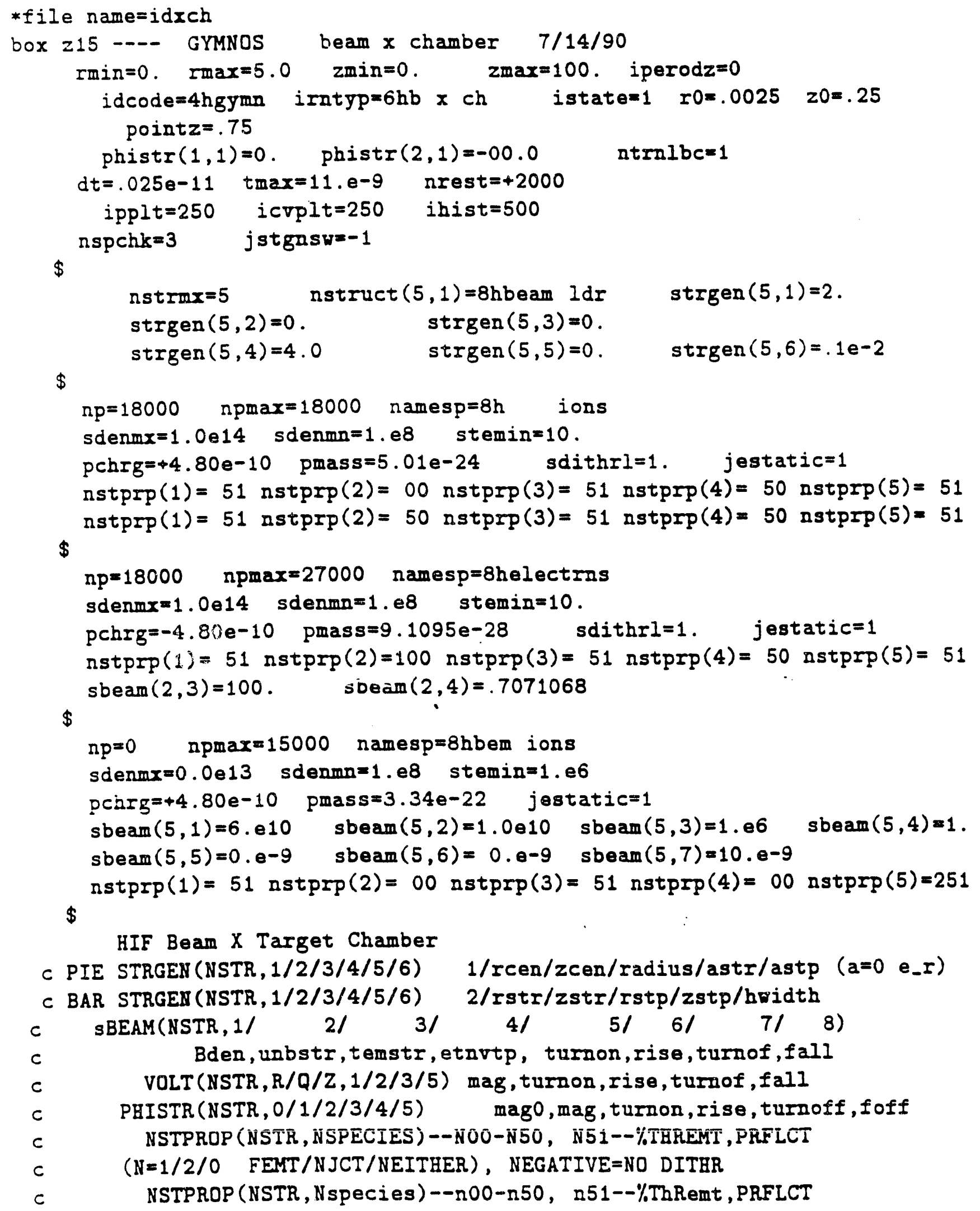


c $(n=1 / 2 / 0$ Femt $/ \mathrm{Njct} /$ Neither $)$, negative=no dithr

*file name=inh2s

box $215-\cdots$ GYMNOS 1Dinhom $T-S \quad 2 / 08 / 90$

$r \min =0.499 \quad \mathrm{max}=0.5 \quad z \min =0 . \quad z \max =.4 \quad$ iperodz $=0$

idcode $=4$ hgymn irntyp $=6$ hnhom $2 \mathrm{~s} \quad$ istate $=2 \quad r 0=.0025 \quad z 0=.25$

phistr $(1,1)=0$. phistr $(2,1)=-00.0 \quad$ ntralbc $=1$

$d t=.010 e-12 \quad t \max =10 . e-12$ nrest $=+1000$

ipplt $=500$ icrplt $=500 \quad$ ihist $=2000$

nspchk $=4 \quad$ jstgnsw $=-1$

$\$$

$\begin{array}{lrr}\text { nstrmx }=5 & \text { nstruct }(5,1)=8 \mathrm{hbeam} l d r & \operatorname{strgen}(5,1)=2 . \\ \operatorname{strgen}(5,2)=0 . & \operatorname{strgen}(5,3)=+0 . & \\ \operatorname{strgen}(5,4)=77.0 & \operatorname{strgen}(5,5)=+0.0 & \operatorname{strgen}(5,6)=.1 \mathrm{e}-2\end{array}$

$\$$

$\mathrm{np}=2000 \quad \mathrm{npmax}=2000$ namesp $=8 \mathrm{~h}$ ions

sienmx $=5.0$ e14 sdenmn=1.e8 stemin=100.

pchrg $=+3.84 e-8 \quad$ pmass $=3.34 e-22 \quad$ sdithrl $=0 . \quad$ jestatic $=1$

$\$$

nstprp $(1)=51 \mathrm{nstprp}(2)=51 \mathrm{nstprp}(3)=50 \mathrm{nstprp}(4)=50 \mathrm{nstprp}(5)=50$

$n p=2000 \quad$ npmax $=2000$ namesp $=8$ helectrns

sdenmx $=4.0$ e16 sdenmn $=1 . e 8 \quad$ stemin $=100$.

pehrg $=-4.80 e-10$ pmass $=9.1095 e-28 \quad$ sdithrl=0. jestatic $=1$

$\$$

nstprp $(1)=51 \mathrm{nstprp}(2)=51$ nstprp $(3)=50 \mathrm{nstprp}(4)=-100 \mathrm{nstprp}(5)=50$

np $=0 \quad$ npmax $=5000$ namesp $=8 \mathrm{hbem}$ ions

sdenmx $=0.0$ e13 sdenmn=1.e8 stemin=1.e6

pchrg $=+9.60 \mathrm{e}-9$ pmass $=3.34 \mathrm{e}-22$ jestatic $=1$

sbeam $(5,1)=6$. e $13 \quad \operatorname{sbeam}(5,2)=1.0010 \quad \operatorname{sbeam}(5,3)=1$. e6 $\quad$ sbeam $(5,4)=1$.

$\operatorname{sbeam}(5,5)=0 . e^{-9} \quad \operatorname{sbeam}(5,6)=0 . e^{-9} \quad \operatorname{sbeam}(5,7)=10 \cdot e^{-9}$

$\$$

nstprp $(1)=51 \operatorname{nstprp}(2)=51$ nstprp $(3)=50$ nstprp $(4)=00 \operatorname{nstprp}(5)=-\Sigma \Xi 1$

np=0 npmax $=5000$ namesp $=8 \mathrm{hbem}$ elec

sdenmx $=0.0 e 15$ sdenmn $=1 . e 8 \quad$ stemin $=100$.

pchrg $=-4.80 e-10$ pmass $=9.1095 e-28 \quad$ jestatic $=1$

sbeam $(5,1)=1.2 \mathrm{e} 15 \quad \operatorname{sbeam}(5,2)=1.0 e 10 \quad \operatorname{sbeam}(5,3)=100 . \quad \operatorname{sbeam}(5,4)=1$.

$\operatorname{sbeam}(5,5)=0 . e^{-9} \quad \operatorname{sbeam}(5,6)=0 . e^{-9} \quad \operatorname{sbeam}(5,7)=10 \cdot e-9$

nstprp $(1)=51 \mathrm{nstprp}(2)=51 \mathrm{nstprp}(3)=50 \mathrm{nstprp}(4)=00 \mathrm{nstprp}(5)=-251$

$\$$

Inhomogeneous Target Two Stream

*file name=iatsa

box z15 --. GYMnnS I.BL Source 12/18/90

Imax $=10.00762 \operatorname{Imin}=9.99746 \mathrm{zmin}=0 . \quad z \max =.00635$ iperodz=0

idcode $=4 \mathrm{hgymn}$ intyp $=4 \mathrm{hrlbl}$ istate $=3 \quad \mathrm{r} 0=.0025 \mathrm{z} 0=.25$ 


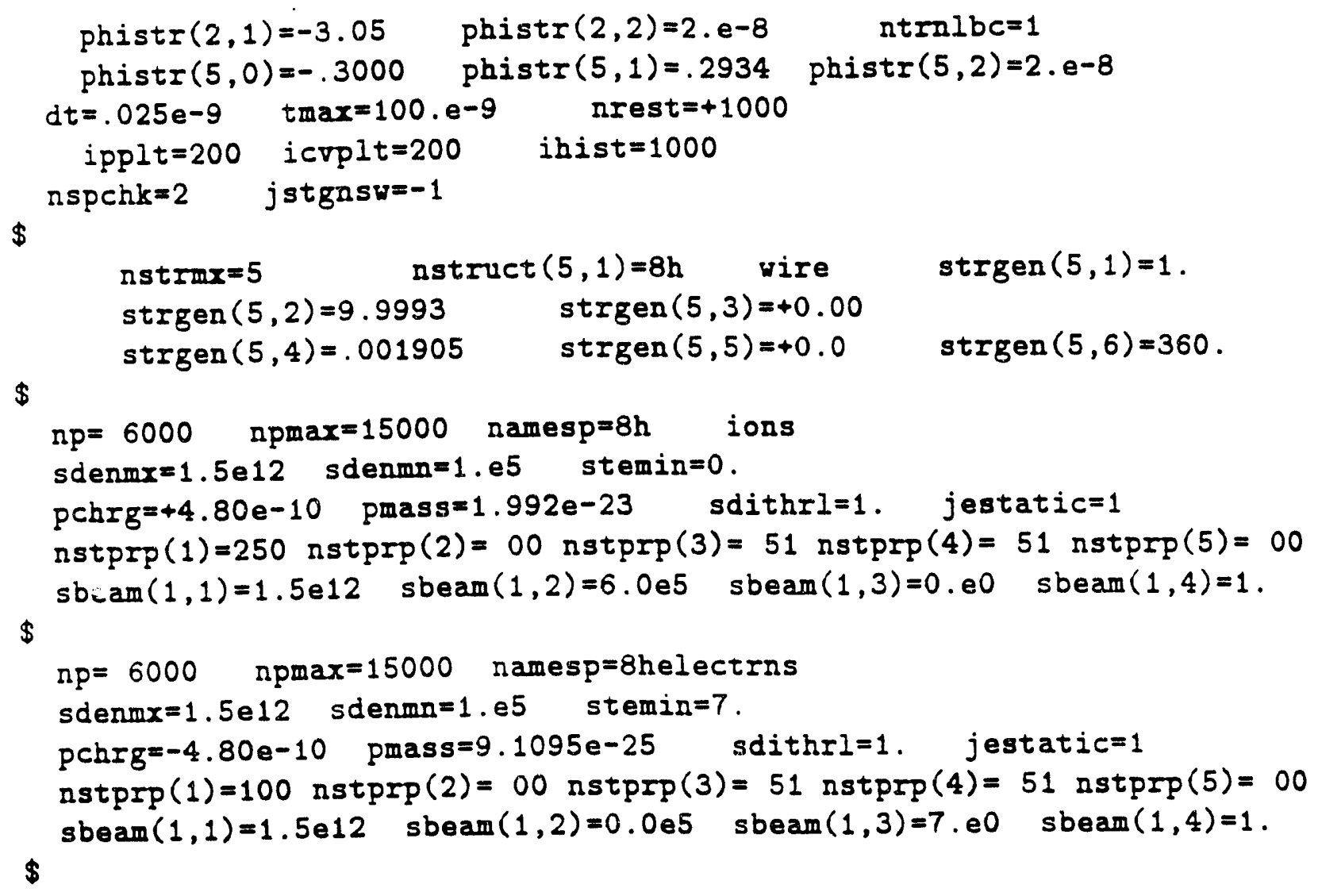

Included below some of the parameter statements used the code when running with the above input decks.

c (prtcl parameters and buffer arrays) PRCOM

Inhomogeneous two stream runs NH2S

c parameter ( $n$ headb $=10$, mpbuf $=1000$, dimp $=5$, memoly=71000,nsp=4) NB2S Ion source runs RLBL

c parameter (nheadb $=10$, mpbuf $=1000$, dimp $=5$, memoly $=151000, n s p=2$ ) RLBL

Beam across chamber runs BXCH

C parameter (nheadb $=10$, mpbuf $=1000$, dimp $=5$, memoly $=301000, n s p=3$ ) BXCB

c (cell parameters and work arrays) CELCOM

c parameter ( $\mathrm{nr} 2=3, \mathrm{nz2}=2001, \operatorname{mrg} 1 \mathrm{~d}=2001, \mathrm{nstmax}=8$, ithmx $=250$,

c. $k \cos m=4385$ ) NH2S

c parameter ( $n r 2=19, n z 2=73, \operatorname{mxg} 1 d=73, n s t m a x=8, i$ thm $=2000$,

c. $k \cos m x=250)$ BXCH

c parameter ( $\mathrm{nr} 2=146, \mathrm{nz2}=19, \operatorname{mxg} 1 \mathrm{~d}=146, n s t m a x=8$, ithmx $=2000$,

c. $k \cos m x=700)$ RLBL 


\section{Appendix 5. WRSTART}

The RESTART subroutine will write WSTART a restart file that contains everything needed to restart RSTART from that file (given the original input deck) and continue the run as if the interuption had never occured. This is almost a requirement for a production code. that may run many hours, to allow decision points about the need to run further, to prodect against machine failures, to allow the addition of more diagnostics, or change the frequency of the plots. Restarts are nearly indispensible for debugging problems that run significant CPU time before they experience difficulty.

In the routine RESTART, there are entries for both reading and writing these files. As you can see from the comments, the routine automatically provides two layers of backups as a hedge against losing everything-requiring the run be started over from scratch. The variables that are saved in these files can be adjusted by moving them in or outside of the locator flags that are used for the starting and stopping points of the BUFFER IN/OUT routines used for the variables in common blocks. For example, all variables stored between MN1First and MN1Last are included in the restart file and thus CANNOT be changed even though they may appear in the input deck. (Such variables can be changed only dynamically with DDT.)

subroutine restart $(m n 1 f, \operatorname{mn} 11, \operatorname{mn} 2 f, \operatorname{mn} 21, \operatorname{mn} 3 f, \operatorname{mn} 31, \operatorname{mn} 4 f, m n 41, \operatorname{mn} 5 f$, mn51, mn6f, mn61, ipbuf , iparmem, nbspec, mspec, nspec, numrest, nsp) dimension ipbuf (1), iparmem (1),nbspec (nsp), mspec(nsp), nspec(nsp), $\operatorname{mn} 1 f(1), \operatorname{mn} 2 f(1), \operatorname{mn} 3 f(1), \operatorname{mn} 4 f(1), \operatorname{mn} 5 f(1), \operatorname{mn} 6 f(1)$ character $* 8$ rfile, Irfile, idum data rfile,rrfile / $r$ ", "rr"/

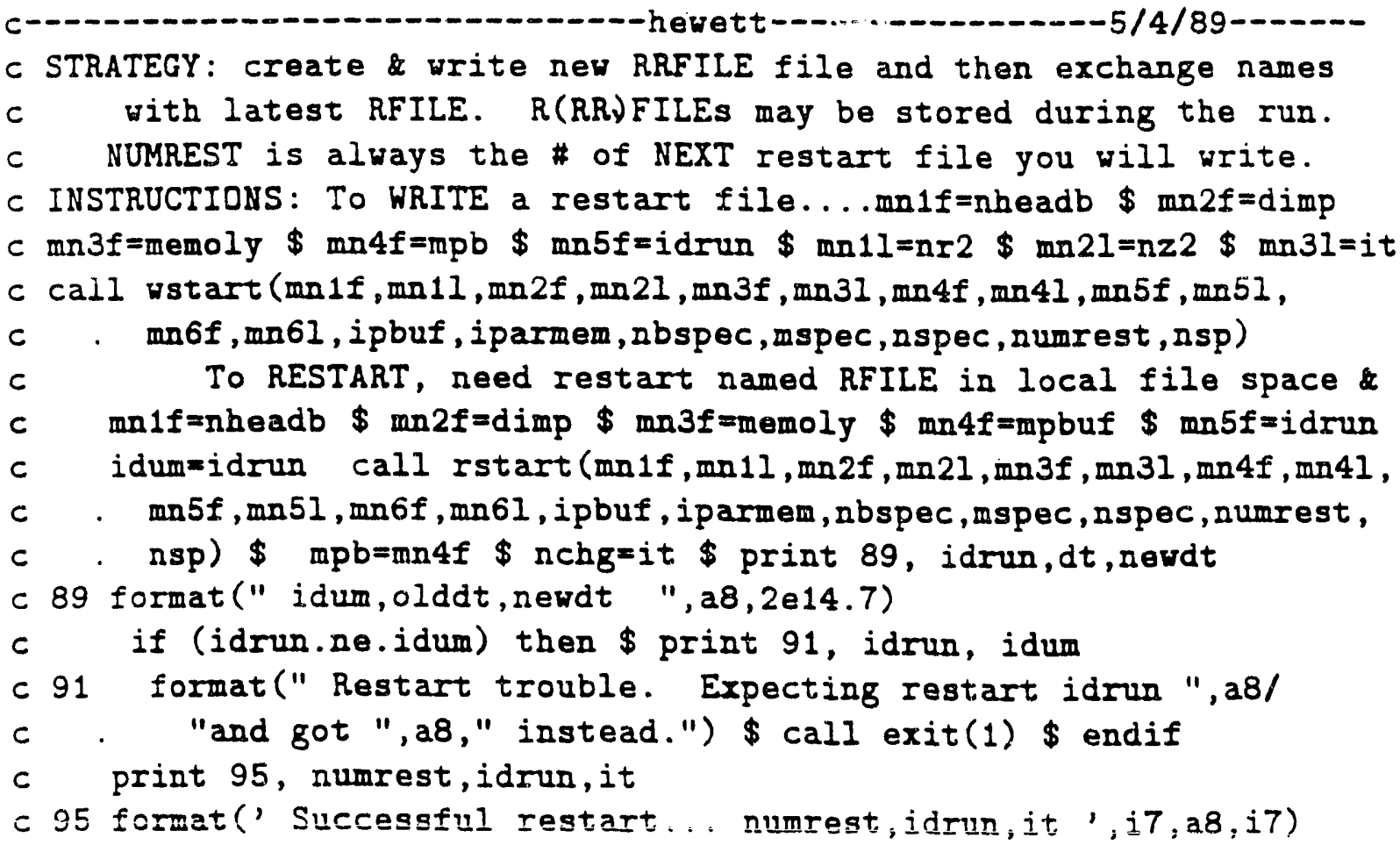




\section{Appendix 6. EFFVOL}

This section discusses the conversion of the "raw particle counts" that PARMOV accumulates at cell corners for each species into particle and current densities. There are two importart issues here-one is a simple geometric consideration and the other is a subtle $r$-dependent systematic error. The systematic error is due to a particle near the small $r$ side of a cell volume contributing the same amount to the cell density as a particle near the large $r$ side of a cell volume. In the limit of a infinite number of particles to represent the plasma, the density loss by particles on the inside is just cancelled by the density gained by those on the outside. The trouble is that on a boundary we only have one type of particle or the other. In particular, at $r=R M I N$ where the error is largest, we only have the outside particles and this systematic error gives rise to density spikes on the axis. if uncorrected. The correction factors, computed in the limit of an infinite number of particles by integration, reduce to the simple forms

$$
R F A C O T=\frac{3(4 R G(I)+D R)}{4(3 R G(I)+D R)}
$$

for cell corners with $L R=+1$ and

$$
R F A C I N=\frac{3(4 R G(I)-D R)}{4(3 R G(I)-D R)}
$$

for cell corners with $L R=-1$.

The geometric corrections are applied in addition to the systematic corrections. The cell corners, where raw counts are accumulated, may be in the 1) plasma region, 2) in a boundary, or 3 ) on a boundaries. If 1 ), just divide by the volume of the associated cell, $\operatorname{VOL}(\mathrm{I})$. If 2 ) it doesn't matter, there will be no particles. If 3 ) we have to consider further possibilities.

For a cell corner on a boundary, we must decide the orientation of the corner. KORNT(I,J) contains all the required information and it is decoded into unit "vectors" LR and $\mathrm{LZ}$ that are $+(-) 1$ if the direction into the plasma region is in the +(-)direction. If we are simply on a edge, say $L R=0$ and $L Z=+1$ for a point on the bottom or ZMIN boundary, then we wish to divide the particle count at that corner by one half the normal cell volume at that radius $\operatorname{VOL}(\mathrm{I})$. If the point is $L R+=1$ and $L Z=0$, then we must divide by the outside part of the cell volume. Similar considerations apply for cell corners but now we must distinguish between "inside" and "outside" corners. In the case of an outside corner, the cell volume is roughly $3 / 4$ of VOL(I) and only roughly $1 / 4$ for the inside corner. Of course the usual $\mathrm{r}$ corrections are employed to correct for the outside cell volume being slightly larger than the inside cell volume.

Now that the reader knows what to expect, it should be easy to pick out the various parts of the effective volume EFFVOL calculation in the Fortran below.

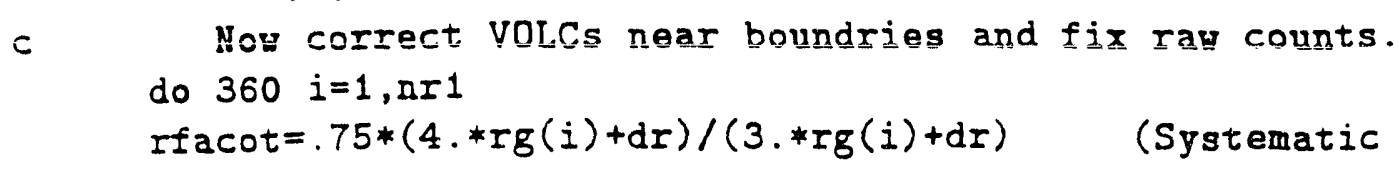




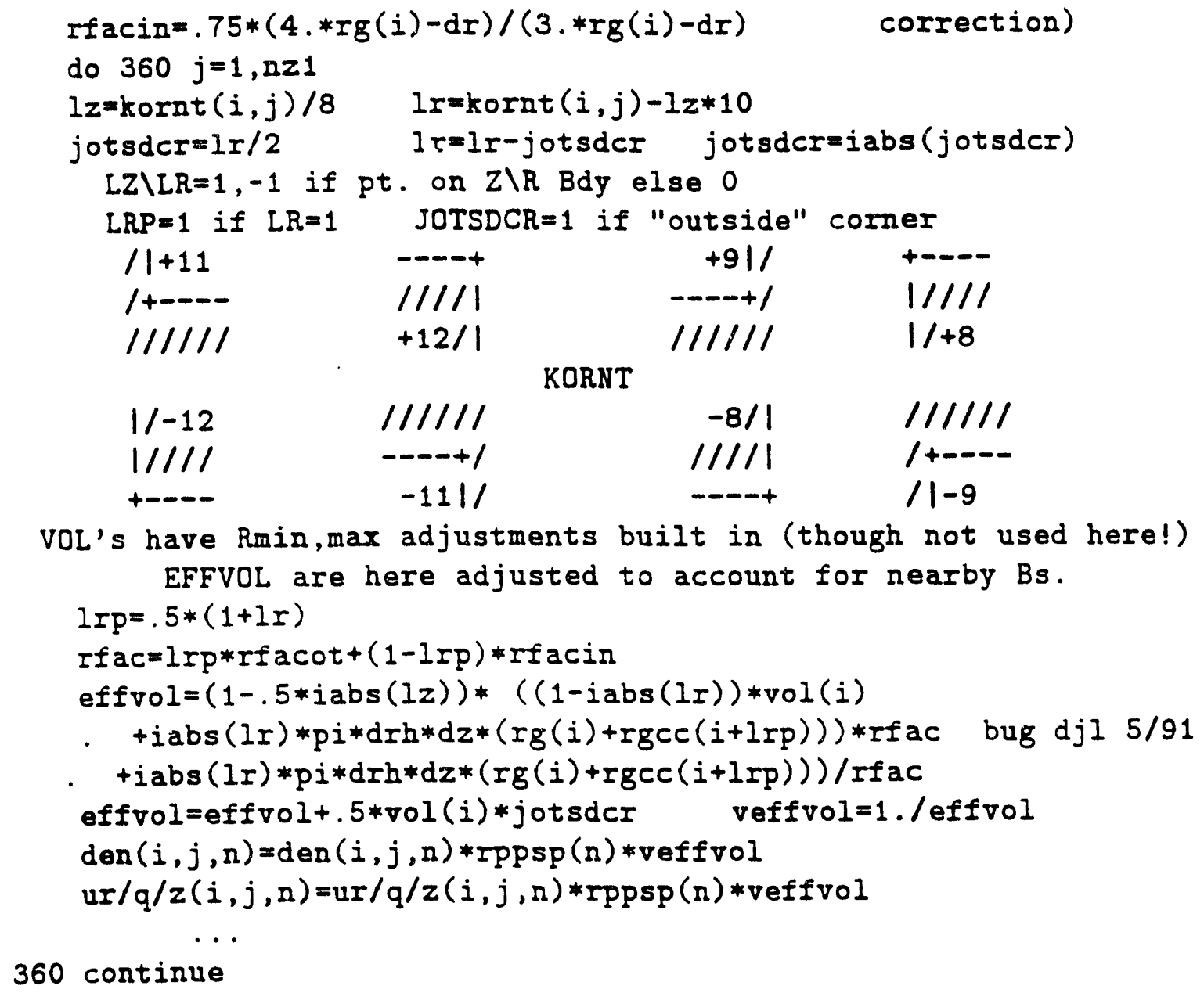




\section{Appendix 7. AUTOVLM}

The AUTOVLM provides automatic scaling for the particle phase plots during the course of a run. The plan is to store in $\operatorname{VZLIM}(1, \mathrm{~N})$ the smallest VZ of any particle of species $\mathrm{N}$ on the time step such prior to the time on which the phase plots are made.. VZLIMI (2,N) The contains the largest VZ, and so on for the VR and VQ components. AUTOVLIM provides a Jduring the time step on which the plotting actually occurs. Several options exist and are coded into the parameter AUTOVLIM. Options available are given in the comments. This coding is all found in TRANS though there is some default initialization in INIT not shown here.

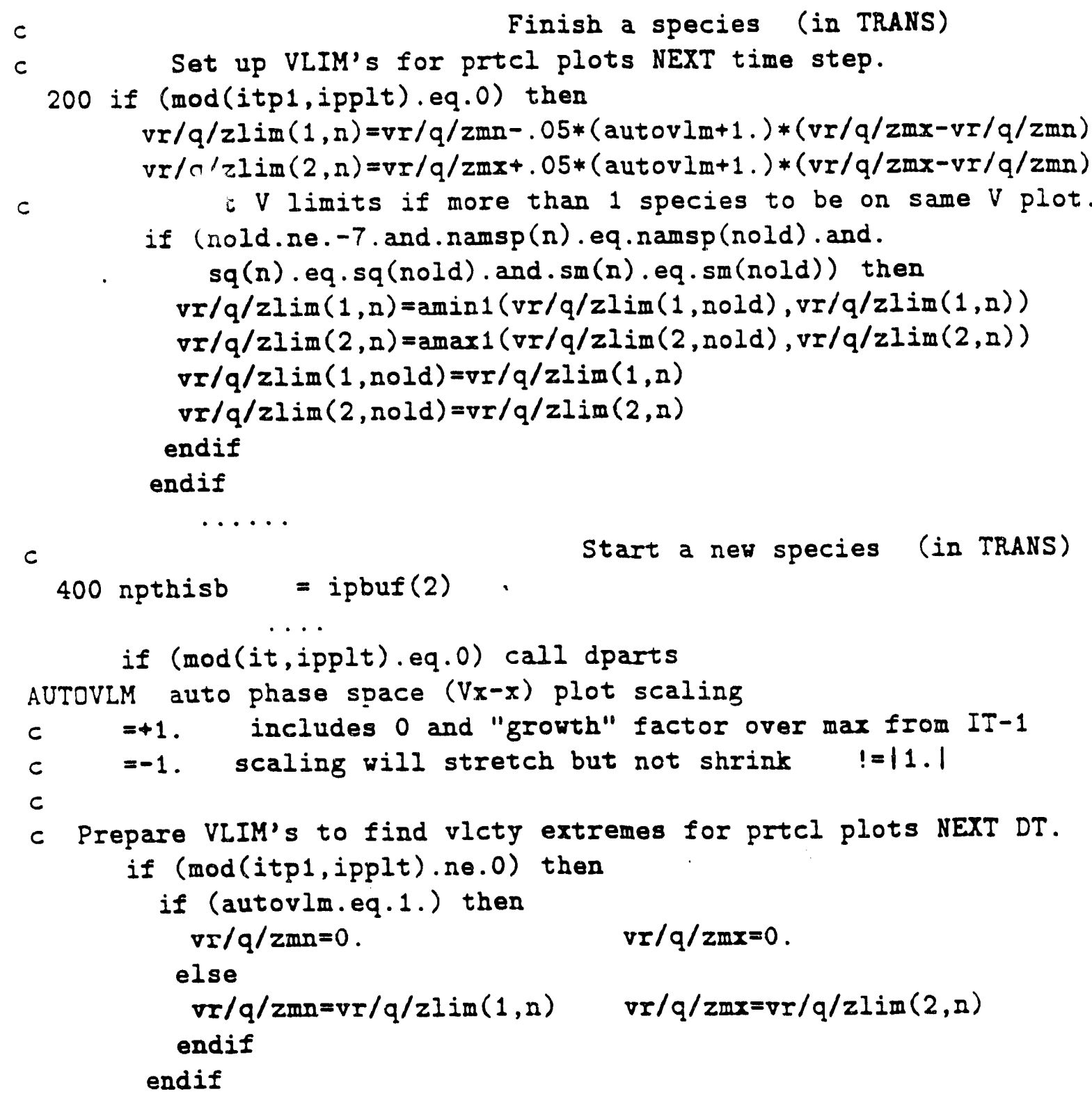




\section{Appendix 8. Particle Buffering Routines: SETB, GETB, RESETB}

The particle buffering routines are set up to do a reasonable job of managing the particles without the user having to know very much about the process. Since memorys are big these days, those of you with short memories may not know that not too long ago it was important to be able to buffer particles to disk files. This package allows for that possibility but, generally, all particles are stored in memory.

The plan is that once the code knows how many species you want, the maximum number of particles of each species, how many quantities per particle, and the desired maximum number of of particles per buffer, the user calls SETB. SETB determines the number of buffers for each species NBSPEC among other things. Subsequent calls to GETB then write buffers to storage as needed and get new buffers to be processed as consistent with the information in NBSPEC. Examples abound in this manual.

These particle quantities are stored serially

$$
(R 1 . Z 1 . V r 1, V q 1, V z 1, R 2, Z 2, V r 2, V q 2, V z 2, R 3 . Z 3 . V r 3, \ldots \ldots)
$$

in the 2D array PBUF. [Again. throughout this write up and GYMNOS. Q or $q$ is used to denote theta.] This storage scheme allows the particles to be managed with the loop index that skips through the loop index as follows:

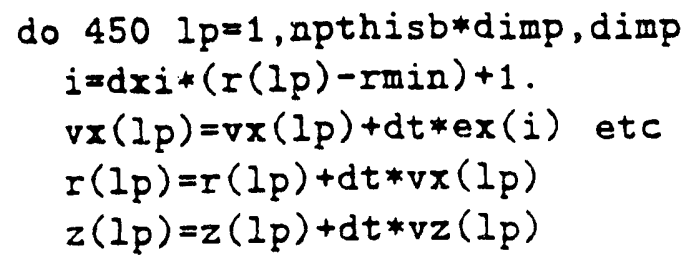

More of the comments found in these buffering routines are given below.

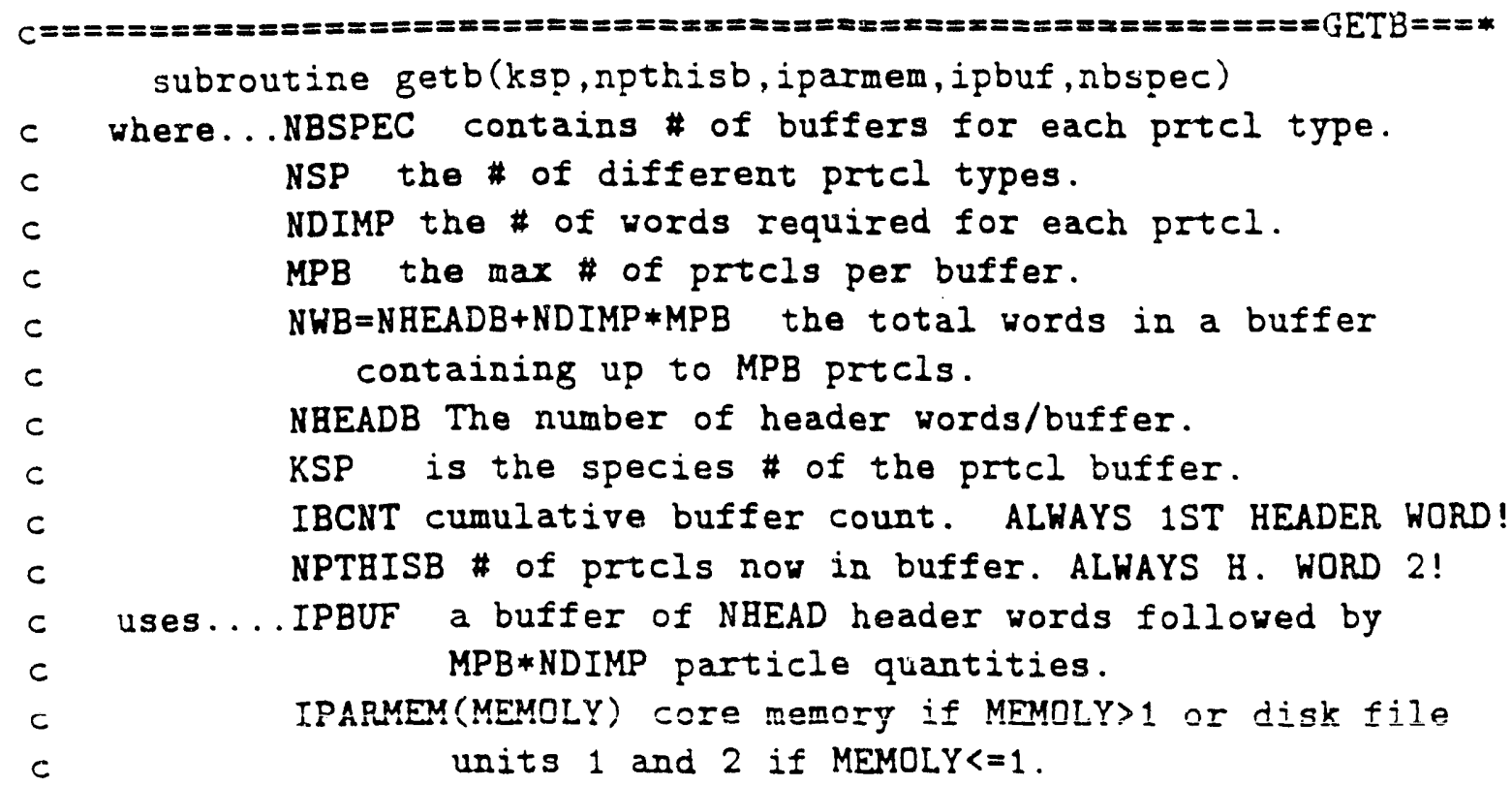


c For storage on disk, just set MEMOLY $=1$. For memory (MEMOLY>1),

c you must choose MLMOLY>=sumNSP NBSPEC $(n) *$ (NBEADB+MPBUF *NDIMP)

$c$ where $\operatorname{NBSPEC}(n)=1+((\operatorname{MSPEC}(n)-1) / \operatorname{MPBUF})$. NBSPEC is computed for

$c$ you in SETB where MPBUF may be reduced MPB for disk.

c

c GENERAL: This two routins do particle buffering. SETB sets up

$c$ buffering \& GETB cycles through the particles as in triple prtcl

$c$ memory buffering. This procedure is similar to Nielson's triple

c prtcl disk buffering circa 1973. This buffering package is

c generalized to allow partially filled buffers with "header" words

$c$ on each to give information about the buffer and its contents.

$c$

c USAGE: SETB is called at the beginning of the run to initialize

$c$ parameters used to buffer the pricls. Subsequent calls to GETB

$c$ write into memory or disk files the present buffer--consisting

$c$ of NHEADB buffer-describing header words and NDIMP floating point

$c$ numbers that characterise each of the NPTHISB particles in this

$c$ buffer. Generally GETB writes the present buffer and reads the

$c$ next. NP!ISB must be consistent with the present buffer; it will

$c$ be reset for the new bufier automatically. KSP contains the new

$c$ species \# on return. If all the buffers of the present species

$c$ \{the \# of buffers for each species is contained in NBSPEC(KSP)\}

$c$ have been processed, KSP is incremented and the first buffer of

$c$ the next species is read into the local nemory IPARMEM. If all

$c$ the buffers for the last species have been piocessed, $K S P=-1$ and

$c$ control is returned to the calling routine.

$C$

c DETAILS: JaII SETB (NSP,MPB,MSPEC, NSPEC, NBSPEC,MEAOLY,DIMP, NHEADB)

$c$ initializes the routines. MPB starts as MPBUF (maximum dimensioned

$c$ number of particles in PBUF) and is used in the calling routine.

c If MEMOLY>1 burfering MPB will be reduced to the largest \# of

$c$ prtels + NBEADB that will fit sito 2 disk sectors.

c Call RESTB (KSP,NPTHISB, iparmem, ipbuf, nbspec) is ved before a

$c$ bufr cycle goes through all bufrs for each species to arrest the

$c$ buffering cycle. For MEMORY BUFFERING (MEMOLY>1), this resets

c parameters so that next call GETB ignores and overurites present

$c$ contents of IPBUF and reads in buffer 1 of species 1 . For DISK

$c$ BUFFERING (MEMOLY=1), call causes everything to be reset as it

$C$ was before this pass started. All particle updates written on

$c$ this aborted pass ignored. (A disk write "pushes" an EOF in front

$c$ of it st if you rewind halfuay out, the EOF is left halfway out.

$c$ Once you a disk file, all information farther out is lost.)

c It is possible to cycle through all particies without writing

$c$ (NPTEISB<0 on input) as is useful in initializing the $t=0$ prtcls. 
Another option is to cycle through particles without reading (KSP $=-2$ on input) for such purposes such as diagnostic passes. SUMMARY. . Never write if NPTHIS $3<0$. Never read if $K S P=-2$.

EXAMPLE

c parameter ( $n$ headb $=10$, mpbuf $=1000$, dimp $=5$, memoly $=80160, n s p=2$ ) 


\section{$c$}

$c$

c

c

c

$c$

$$
\text { c }
$$$$
\text { c }
$$

c Check memory and determine NBSPEC into 2 disk sectors.

Call to SETB initializes the buffering routines. MPB starts as MPBUF (maximum dimensioned number of particles in PBUF) and is used in the calling routine. If MEMOLY>1, MPB will be reduced to the largest number of particles+NHEADB that will fit (see GETB for documentation) $4 / 3 / 89,88,77$ Compute MPB, max \# prtcls that fit in 2 disk sector records.

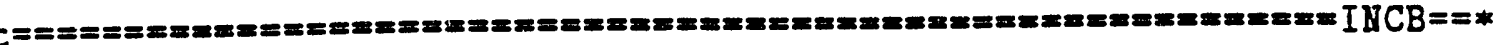
subroutine incb( $n, n b i n c, n b s p e c$, ipbuf, iparmem)

C Call to INCB enlarges \# of bufrs allowed for species $N$ by NBINC.

c Other parameters are documented in GETB.

$c$ where...NBSPEC contains \# of buffers for each prtcl type. NSP the of different prtcl types.

NWB the total words in a buffer

IBCNT cumulative buffer count. ALWAYS 1st HEADER WORD! NPTEISB \# partcls this buffer. ALWAYS 2nd HEADER WORD! uses.... IPBUF a buffer of MWB words.

Memory if MEMOLY>1 or disk files units 1 \& 2 if MEMOLY $=1$. hewett $2 / 90$
$c$
c March through disk file from front, putting extra dumb writes $c$ writes in file after existing NBSPEC(N) buffers of speci:. N.
$c$ Can only do this after a full sweep through all buffers so that
c IT1 \& IT2 have just been switched.
c CORE
c March back towards front of particle core storage, copying the
$c$ trailing buffers NBINC*MWB farther out. Process stops at species
c $N+1$ so that room has been made for the desired extIa NBINC buffers
$c$ for species $N$. Could do this within a full sweop anytime before
c species $N$ is started--like after a RESETB. 


\section{Appendix 9. VDKR}

This is the routine that started the whole GYMNOS project; see the HISTORY section in the introduction. The salient feature is that this routine treats all point, boundary or interior. equally. Any boundary condition can be applied at any point.

And it all vectorizes.

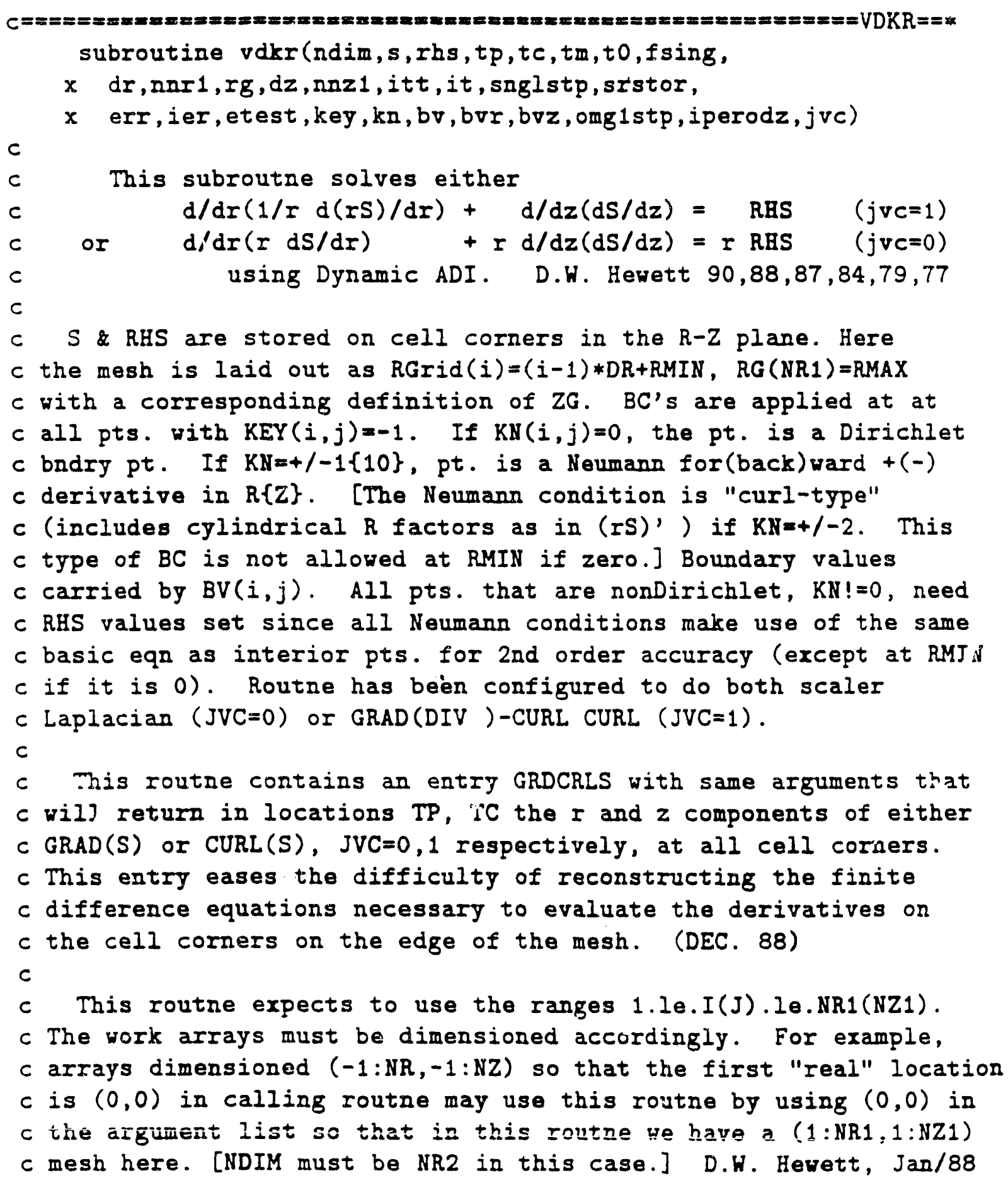

c This version of VDADI uses "KEY"s to construct intrl bndrys. 
Interior

KEY $=+1$ if in the plasma

$=0$ if in a vacuum region

$=-1$ if ON or IN intrl boundry

$\mathrm{KN}=0, \mathrm{BC}$ pt is Dirichlet

$=+1(-1)$ on boundary w/ for(back)ward R-derivative

$=+2(-2)$ curl-type Neumann

$=+8(-8)$ on bdry $w /+(-) Z \&-(+) R$ (curl)-derivative $=+9(-9)$ on bdry $w /+(-) \mathrm{Z} \&-(+) \mathrm{R}$-derivative

$=+10(-10)$ on intIl boundary w/ for(back)ward $Z$-drvtv

$=+11(-11)$ on bdry $/+(-) Z z+(-) R$-derivative

$=+12(-12)$ on bdry $w /(-) Z \&+(-) R$ (curl)-derivative

BV = the value specified on and in the intrl bousiary.

CAUTION: Intrl boundaries are built only at cell edges! Double Neumann pt both use same BVR and BVZ.

$\operatorname{KEY}(i, j)=1=$ plasma $\operatorname{kd}=-\operatorname{key}(i, j) *(1-\operatorname{key}(i, j)) / 2$

omkd=1-kd

$k y=i \operatorname{abs}(\operatorname{key}(i, j))$

$o m k y=1-k y$

$K N(i, j)=10=$ forward $Z$-Numan, $1=$ forkard $R$-Neumann. -2=backward curl $R$-Neumann, $12=$ forw $R$ (curl) $-N$ forw $Z-N$, 9=back $R-N$ forw $Z-N$, $-8=$ forw $R$ (curl) $-N$ back $Z-N$, $-11=$ back $R-N$ back $Z-N$, $k n z=k n(i, j) / 8$ gives $+/-1$ $k n r=k n(i, j)-k n z * 10$ gives $+/-2$ or $k+y p=k n r / 2$ $k n r=k n r-k t y p$ $k t y p=i a b s$ (ktyp) gives $+/-1$ gives $+/-1$ $0=v a c u u i n, \quad-1=B C p t$.

gives 1 on a BC pt. else 0 gives 1 for norbC pt. gives full eq. in plasma on $B C$ gives 1 in vacuum 2=forward curl $R$-Numan, $-10=$ backward Z-Neuman $11=$ forw $R-N$ forw $Z-N$, $8=$ back $R$ (curl)-N forw $Z-N$, $-9=f \circ r w R-N$ back $Z-N$, -12=back $R$ (curl)-N back $Z-N$ for $z$-Neumann else 0 1 for $r$-Neumann else 0 to make r-Neumann KTXP for I-Neuman else 0 for curl-type $R$ Neumann

General BC set up B.sic eqn. $\quad \operatorname{crp} * s(i+1, j)+\operatorname{crm} * s(i-1, j)+c z p * s(i, j+1)+c z m * s(i, j-1)$ $+($ crctcze $\circ$ omg $) * s(i, j)=\operatorname{rhs}-\operatorname{omg} * s(i, j)$ $R$ pass coding $t_{p}=c r p \quad c p=c z p$ $t c=c r c-o m g \quad c c=c z c+o m g \quad t 0=\operatorname{rhs}-c p * s(j+1)-c c * s(j)-c m * s(j-1)$ tm=crm $\quad c m=c z m$ Dirichlet point $K D=1 \quad K N R=K N Z=0$

\section{$K D=K N R=K N Z=0$}

$c p=0$ $c c=0$ $t 0=b v$ 


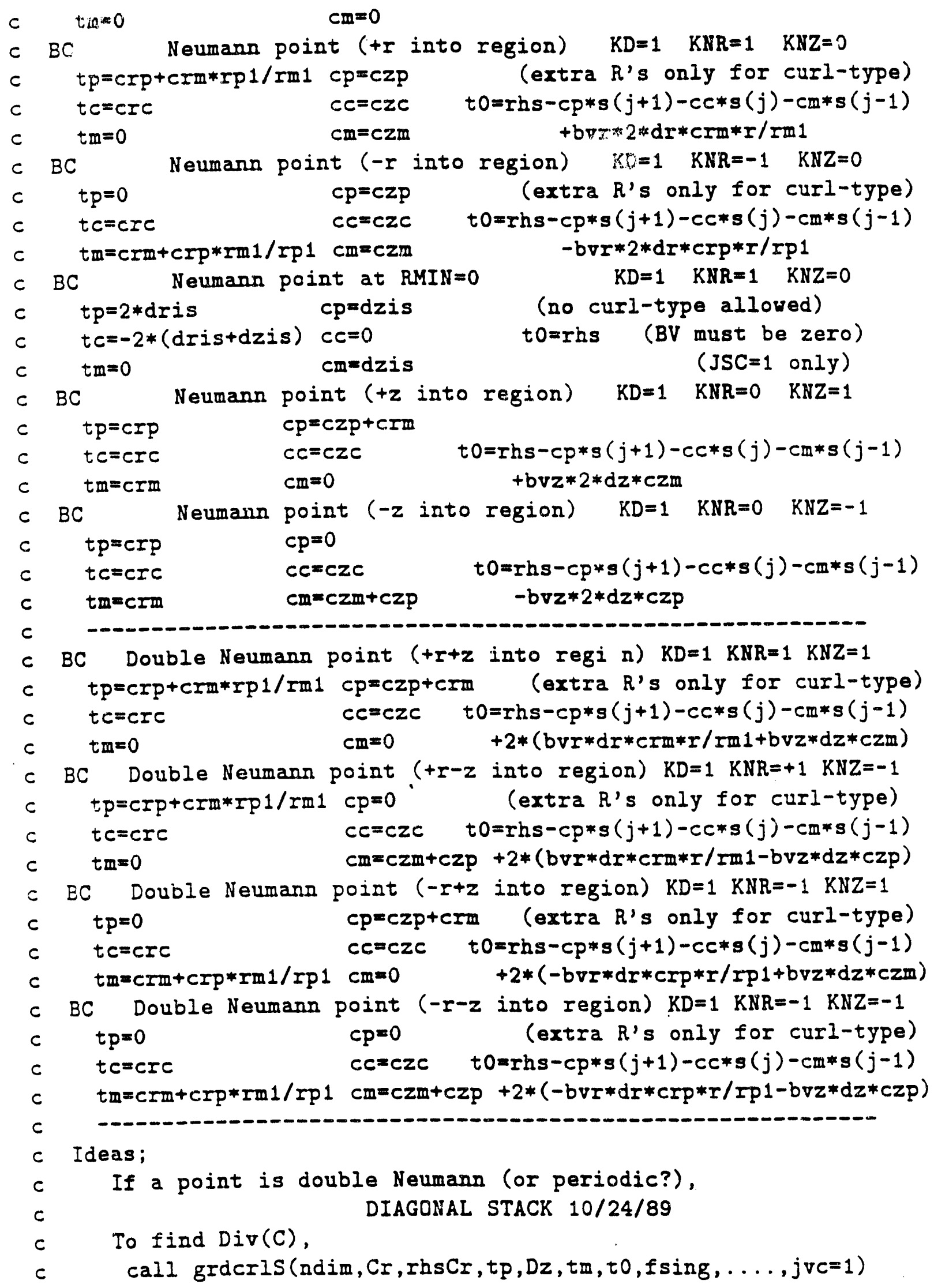




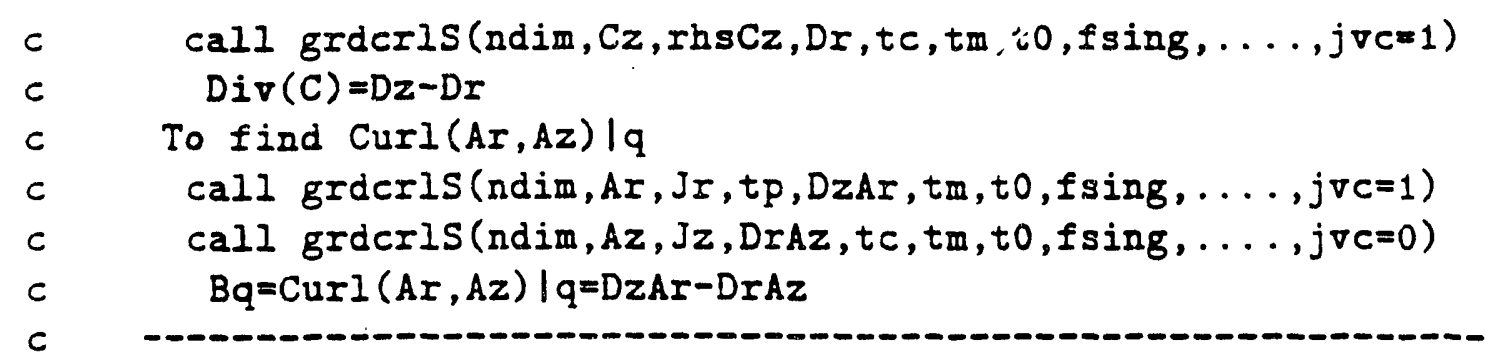

The coding that actually accomplishes the above set up follows. The $z$ pass section is identical except that the definitions for $\mathrm{TP}(\mathrm{I}, \mathrm{J})$ and $\mathrm{CP}, \mathrm{TC}(\mathrm{I}, \mathrm{J})$ and $\mathrm{CC}$, and $\mathrm{TN}(\mathrm{I}, \mathrm{J})$ and $\mathrm{CM}$ are interchanged and the sign of the ADI acceleration factor is changed. In addition Neumann boundary conditons for the scaler Poisson equation require an application of l'Hospital's rule to avoid dividing by zero in the finite difference algorithm if $R M I N=0$. The details are given in the FORTRAN below. It's "straightforward"!

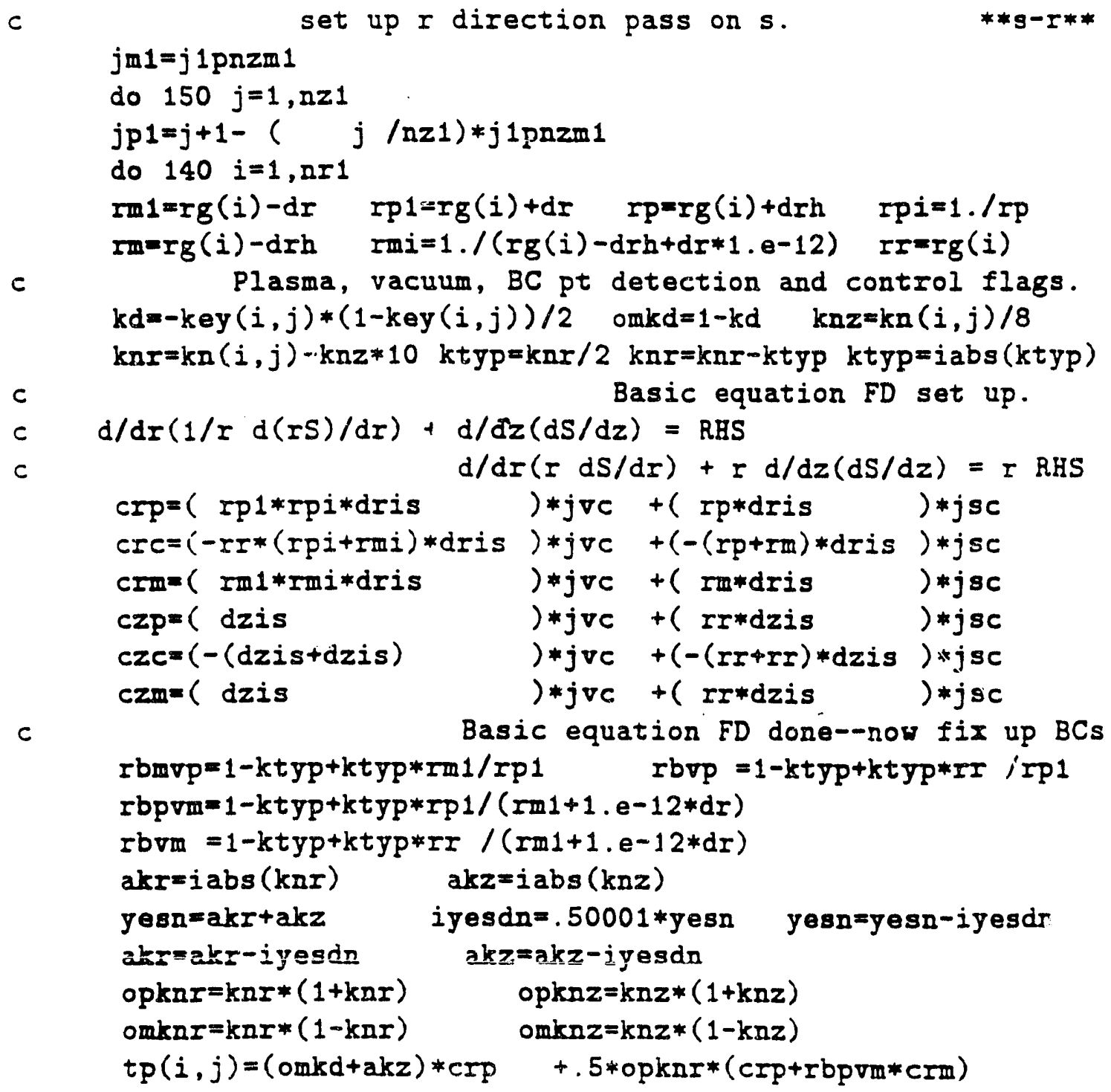




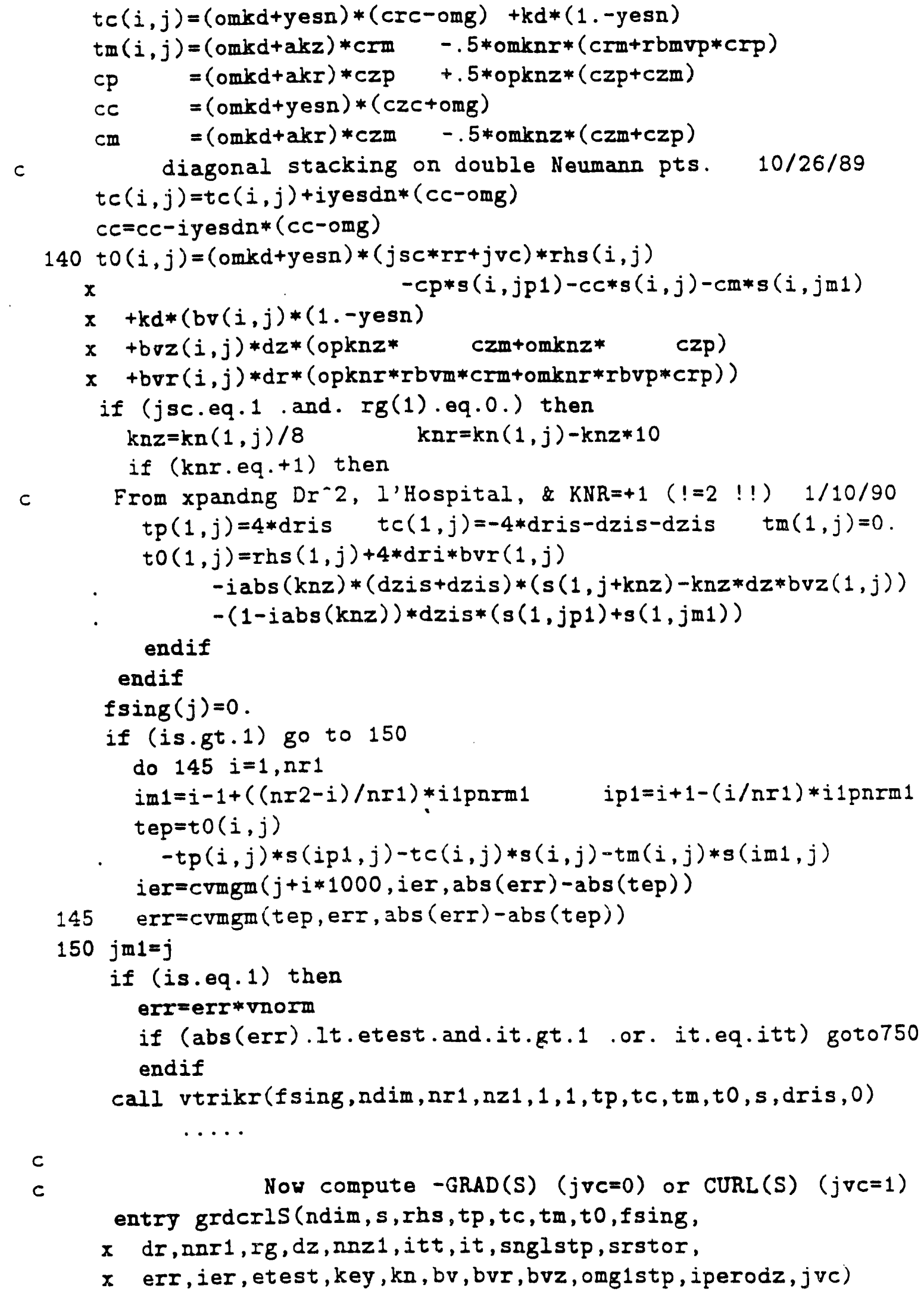

c

c

$$
\text { Now compute }-G R A D(S) \quad(j \nabla C=0) \text { or } \operatorname{CURL}(S) \quad(j \vee c=1)
$$

entry grdcrls(ndim, $s$, rhs, tp,tc,tm,to,fsing,

$x d r, n n I 1, r g, d z, n n z 1, i t t$, it,snglstp,srstor,

$x$ err,ier, etest, key, $k n, b v, b v r, b v z$, omglstp, iperodz, jvc) 
$c$

Vector returned; $\mathrm{R}$-comp in TP...Z-comp in TC 1st, set Values around 4 outside walls (do 850 i \& 825 j)

Basic equation FD set up. $d / d r(1 / r d(r S) / d r)+d / d z(d S / d z)=$ RHS $d / d r(r d S / d r)+r d / d z(d S / d z)=r$ RHS If this mesh edge pt is 1 of the 4 comers, fix it. DIRICHLET CORNER POINT SECTION If corner Dirichlet, assume both bdys are Dirichlet \& do 1-sided differences as if bdys were linear. 10/24/89

Check assumption--If 1 of the Bdys meeting in corner not Drchlt, project Drchlt inearly \& compute Sout in other.

$R$ bdy Drchlt so treat $z$-drvtive as given (as just xtrpolted) \& compute Sout $[=\mathbf{s}(0$ orNR2,10rNZ1)] for r-drvtive as below.

$Z$ bdy Drchlt so treat r-drvtive as given (as just xtrpolted) \& compute Sout [=s(10rNR1, 0orNZ2)] for $z$-drvtive as below. Must have been Dirichlet on both boundaries--pt finished. NEUMANN CORNER POINT SECTION $\mathrm{KNR}=1, \mathrm{KNZ}=0 \quad Z$-derivative known (TP) ... need $R$-der. (TC) $K N R=0, K N Z=1 \quad R-d r v t v$ known.. need $Z$-drvtv. Finish interior $-\operatorname{grad}(s)$ or curI(s) 


\section{Appendix 10. VTRIK}

This routine is a fully vectorized tridiagonal solver. Consider a system of NR1 by NZ1 points. For say the implicit $R$ pass, we have NZ1 lines in the $R$ direction of the overall matrix that need to have a tridagonal matrix solved. Even though the tridiagonal algorithm is inherently recursive on each line. we can still vecotrize by doing all the NZ1 lines at once. VDKR accomplishes this with separate entries for $\mathrm{R}$ and $\mathrm{Z}$ passes and. within each entry, is a switch for producing solutions with periodic boundary conditions.

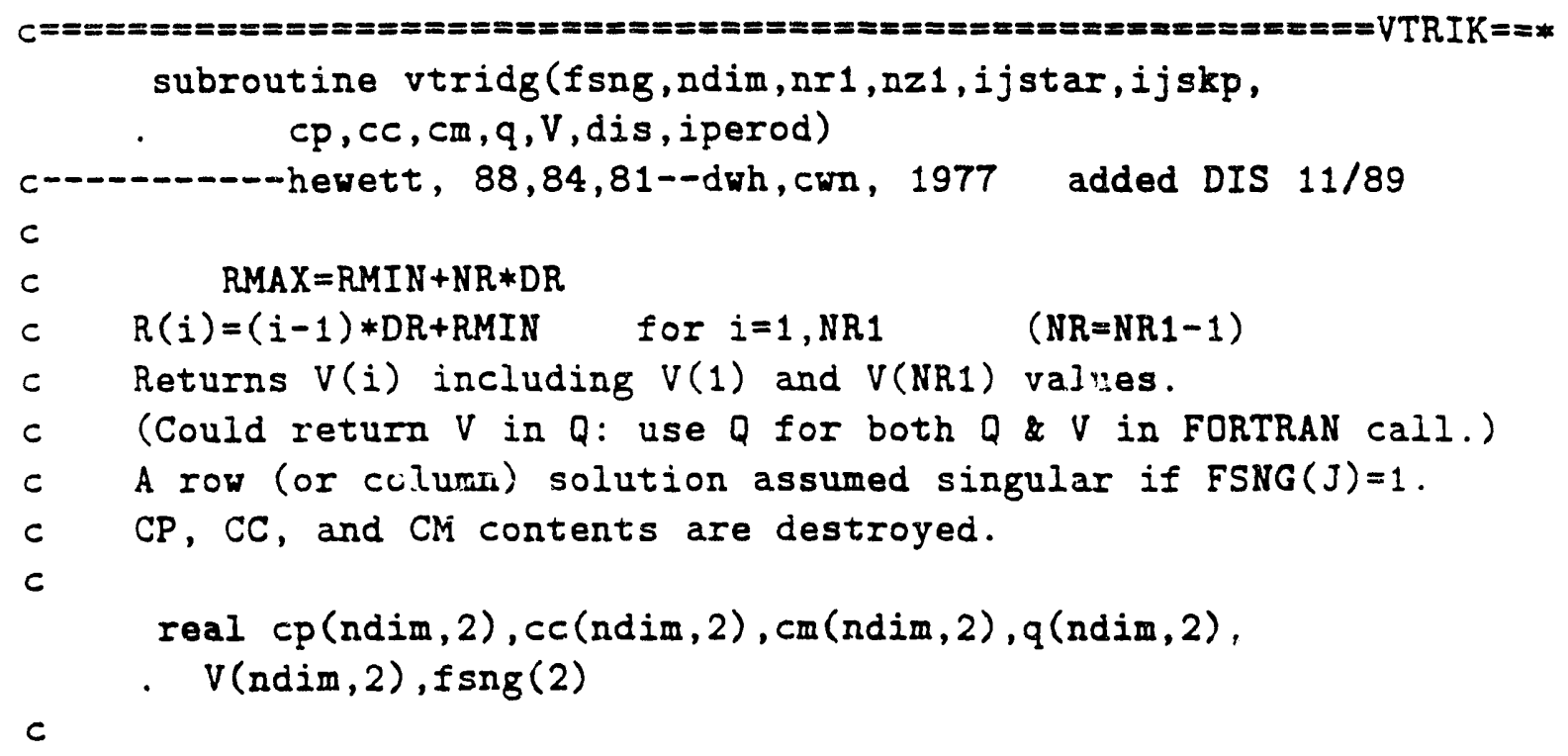

The entry VTRIKR provides the $R$ pass solution. entry vtrikr(fsng,ndim,nr1,nz1,jstar,jskp, $c p, c c, c m, q, V, d i s$, iperod)

This entry solves tridagonal matrices equations

$$
c p(i, j) * V(i+1 . j)+c c(i, j) * V(i, j)+c m(i, j) * V(i-1 . j)=q(i, j)
$$

for J=JSTAR,NZ1.JSKP where JSTAR is a arbitrary first line and JSKP is a skip parameter. (This same routine is often used for red-black line SOR and the starting offset and skipping by 2 makes this convenient.) IPEROD $=1$ declares the system to be periodic; and FSNG(J)=1. declares the system to be singular (e.g. when the solution is arbitrary to within a constant).

The entry for $\mathrm{Z}$ passes is

$$
\begin{gathered}
\text { entry vtrikz(fsng, ndim, } n r 1, n z 1, \text { istar, iskp, } \\
(c p, c c, c m, q, V, d i s, \text { iperod) }
\end{gathered}
$$

and this causes the system of columns

$$
c p(i, j) * V(i, j+1)+c c(i, j) * V(i, j)+c m(i, j) * V(i, j-1)=q(i, j)
$$

to be solved for simultaneously for I=ISTAR,NR1,ISKP. Controls analogous to the $R$ pass are applied via FSNG(I) and IPEROD. 
Appendix 11. VPARMOV/VTRAP/VBIRTH/DEATH

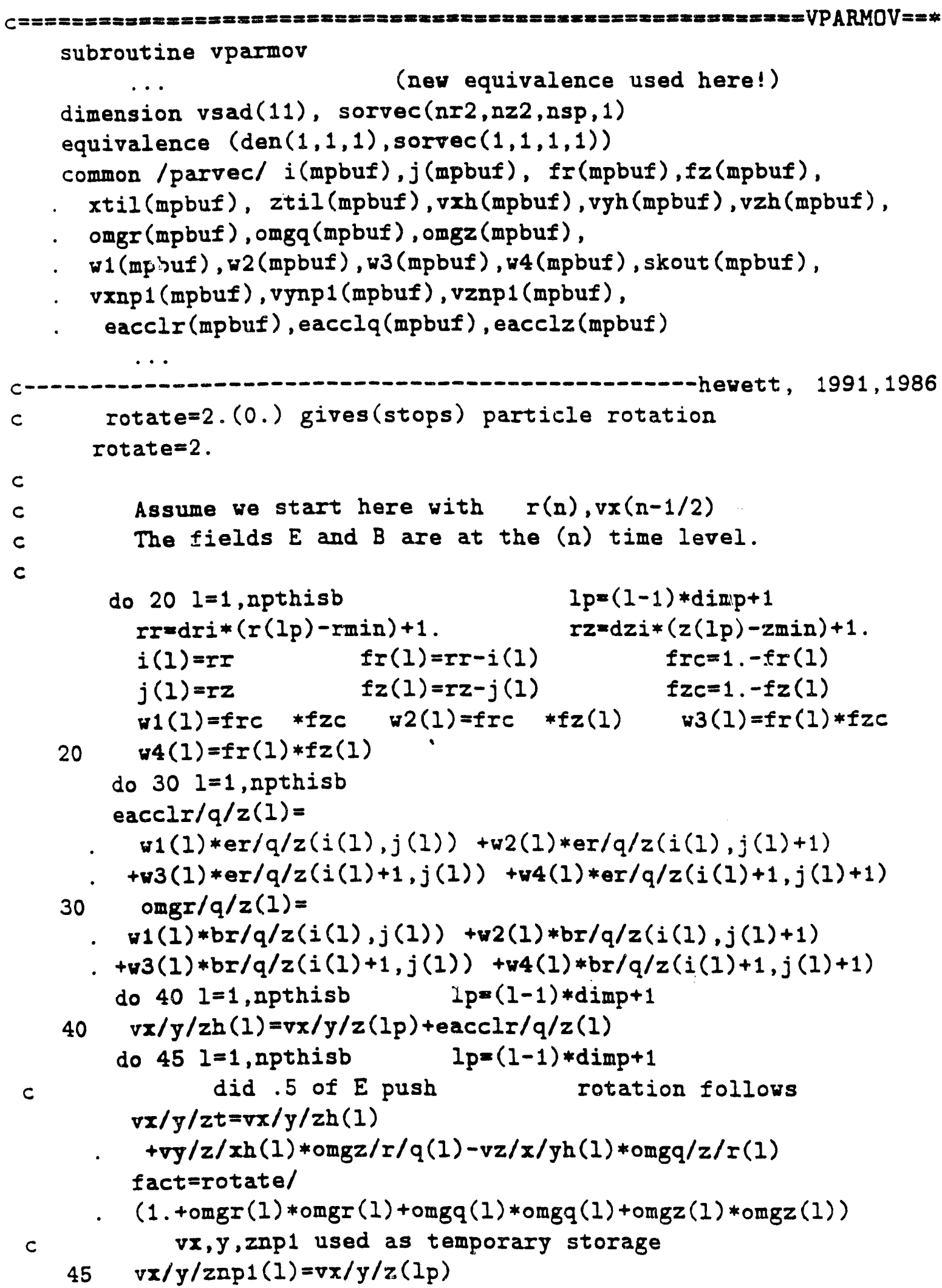




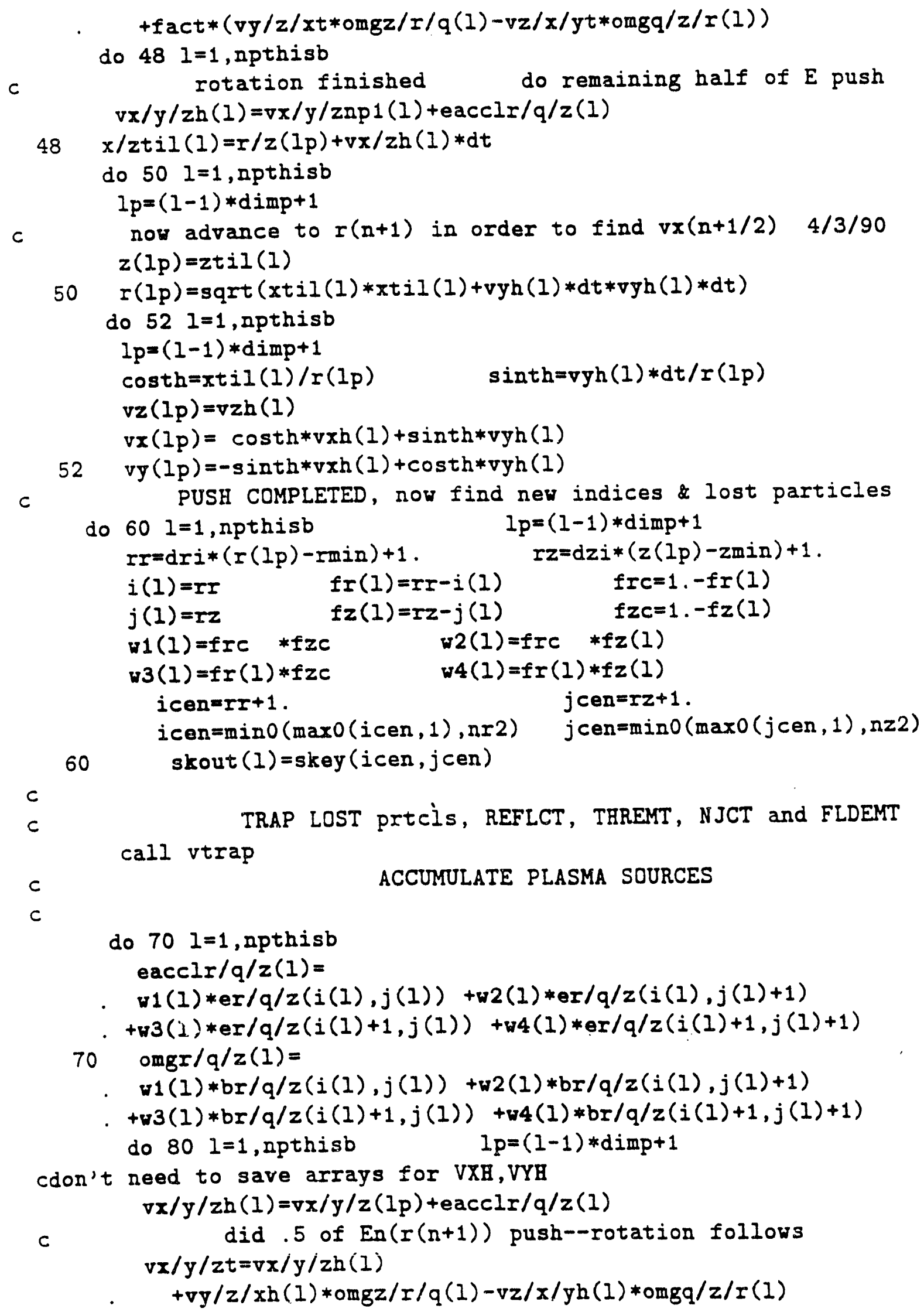




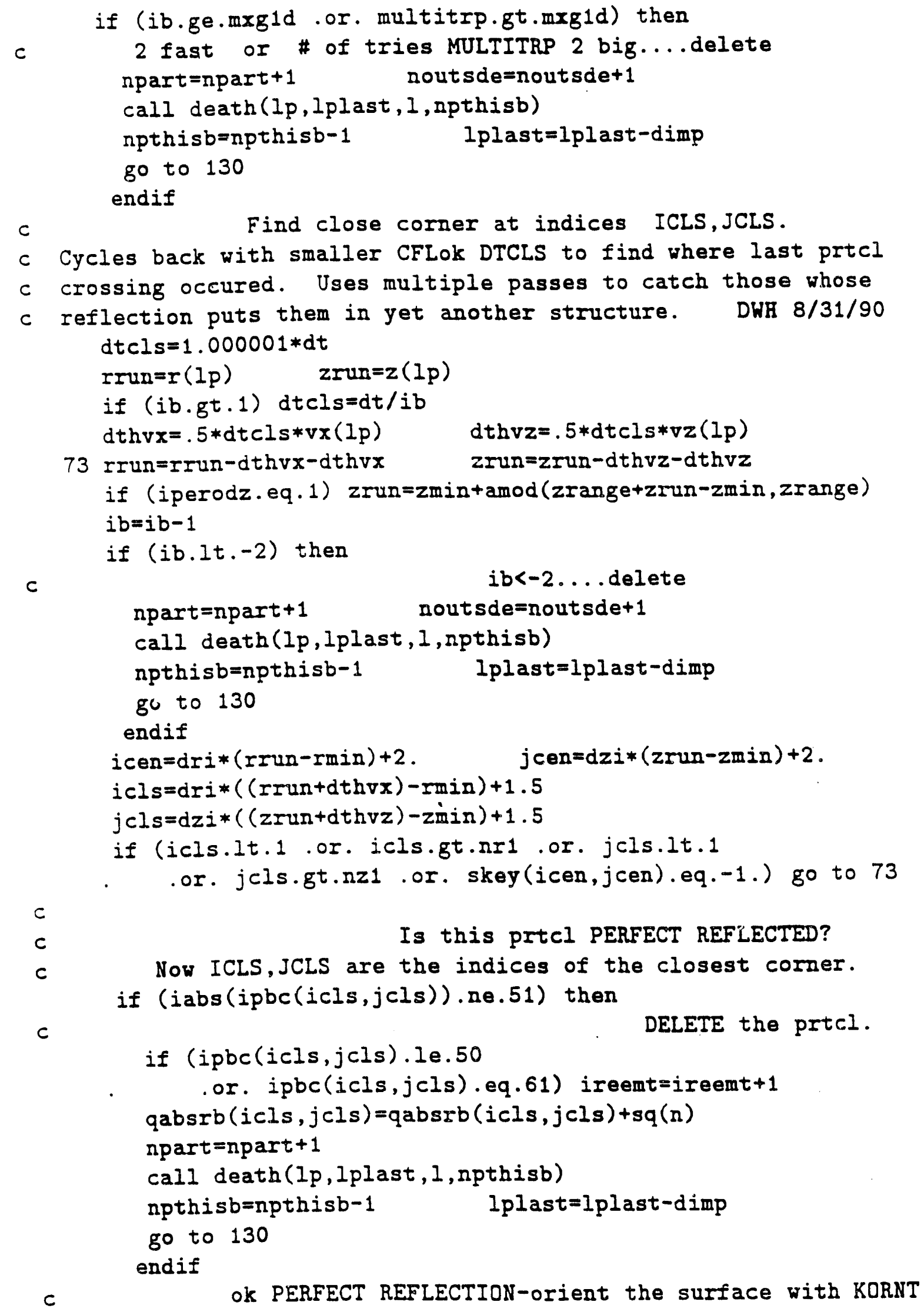




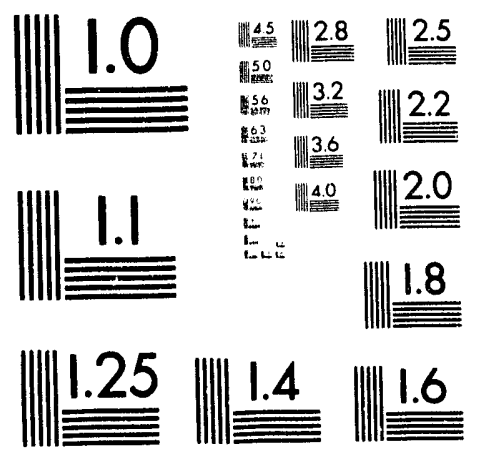



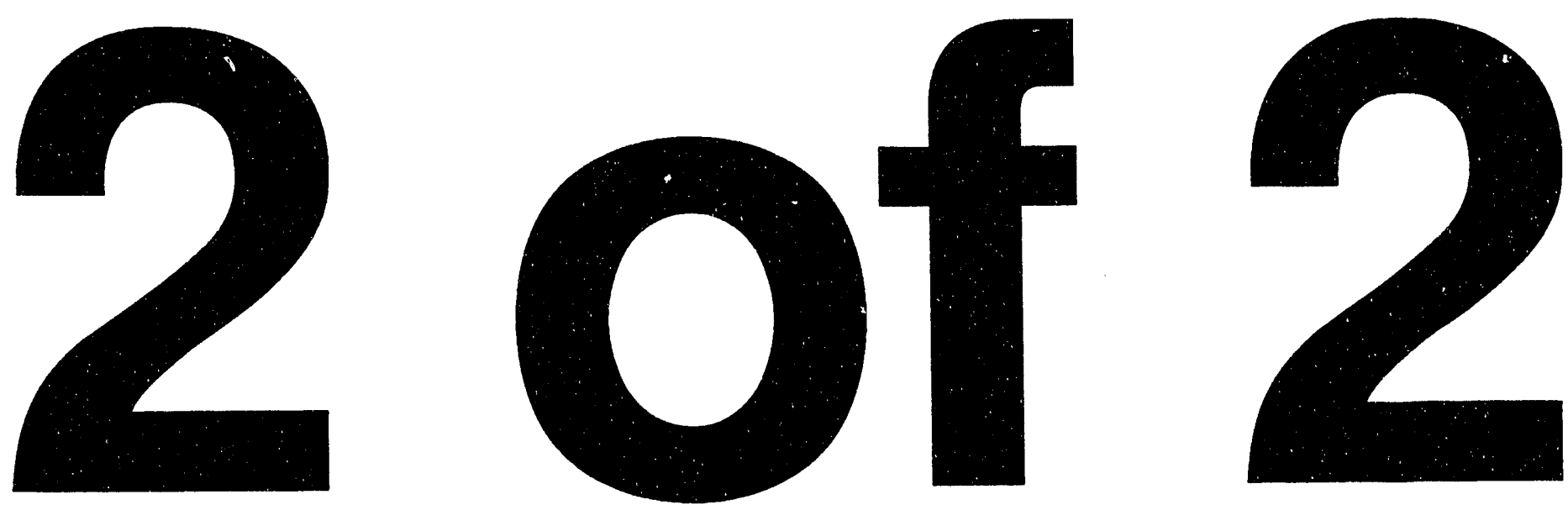
...same as TRAP

$c$

$c$

Now Thermal REEMISSION from cell corners

...same as TRAP

call vbirth (npart, vmp, unorm, evnvth, evtrth, kornt (icor, jcor), edithr, edithz,sgnr,sgnz, Ig(icor), zg(jcor))

...same as TRAP

$c$

c nOW FIELD EMIT OI NJCT NEW prtCls

...same as TRAP

call vbirth(npart, vmp, urorm, evnvth, evtrth, 0 , edithr, edithz, sgar, sgaz, remt, zemt)

...same as TRAP

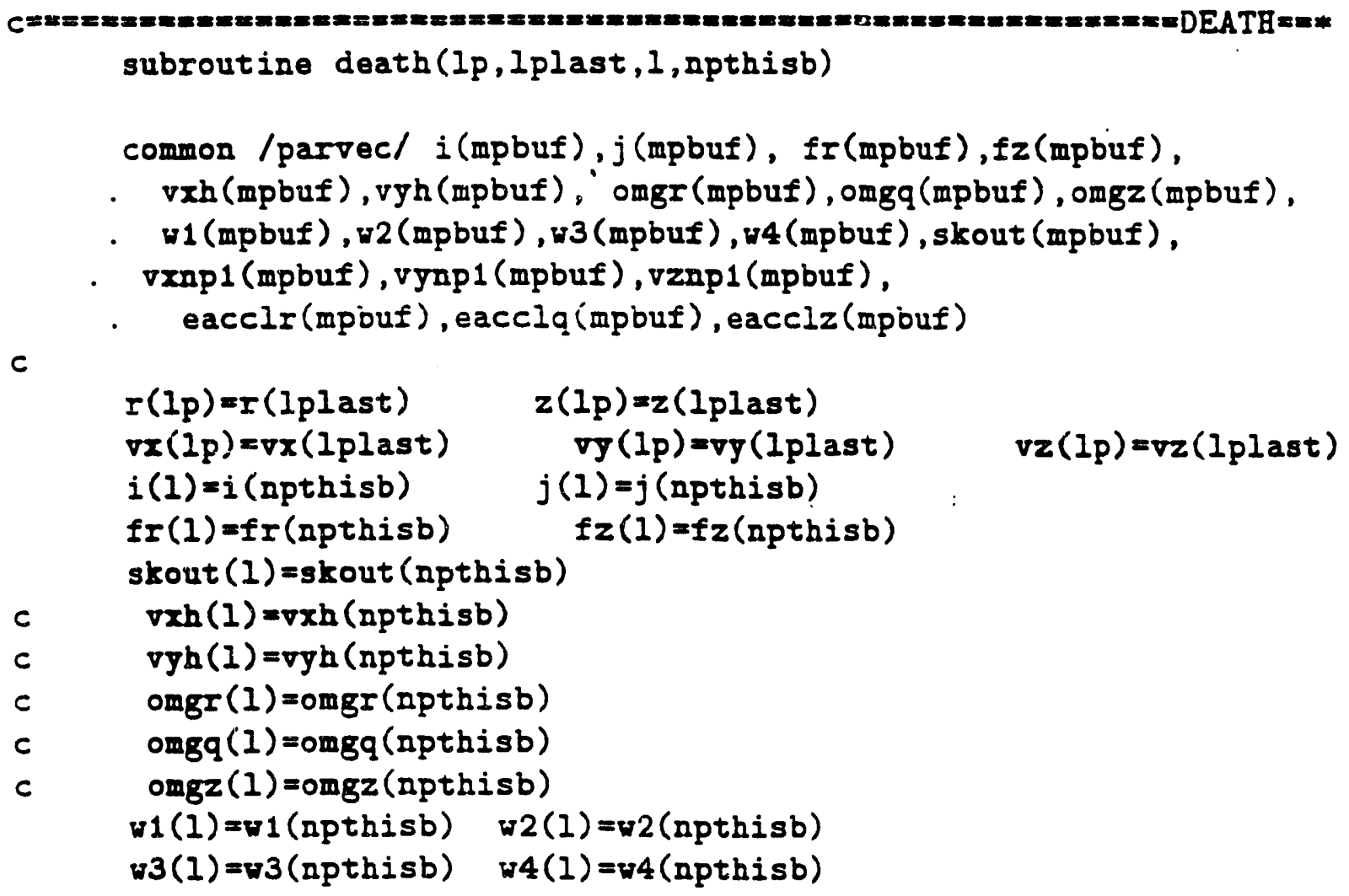

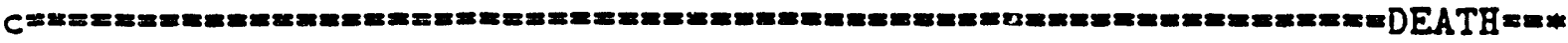

subroutine death(Ip,Iplast,1,npthisb)

common /parvec/ $i$ (mpbuf), $j$ (mpbuf), fr(mpbuf), fz(mpbuf), vxh (mpbuf), vyh (mpbuf), omgr (mpbuf), omgq (mpbuf), omgz (mpbuf),

. w1(mpbuf), w2(mpbuf), w3 (mpbuf), w4 (mpbuf), skout (mpbuf), vxnp1 (mpbuf), vynp1 (mpbuf), vznp1 (mpbuf), eacclr (mpouf), eacclạ (mpbuf), eacclz (mpbuf)

C 


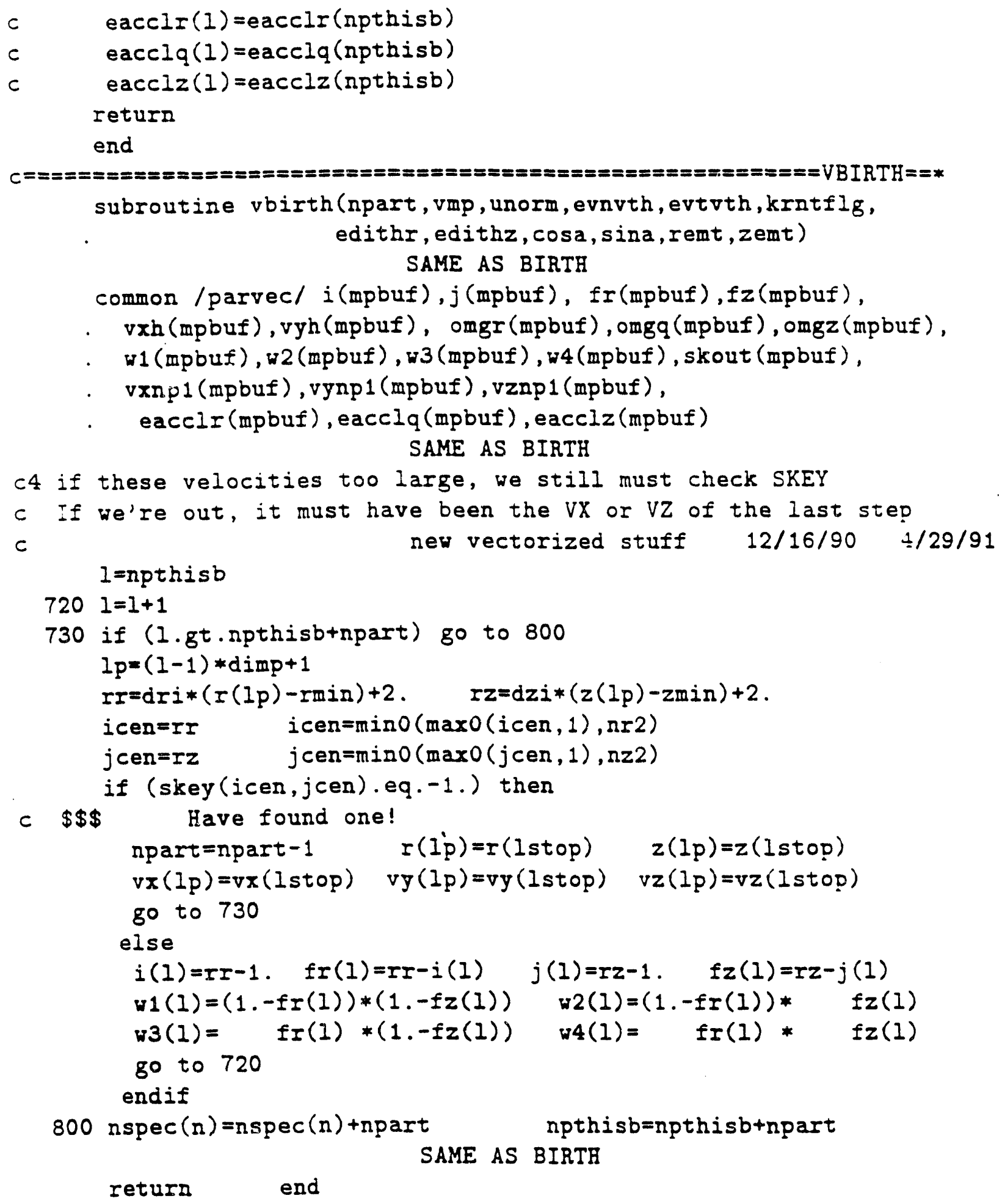




\section{Appendix 12. PARMOVR}

David J. Larson's relativistic modification of PARMOV.

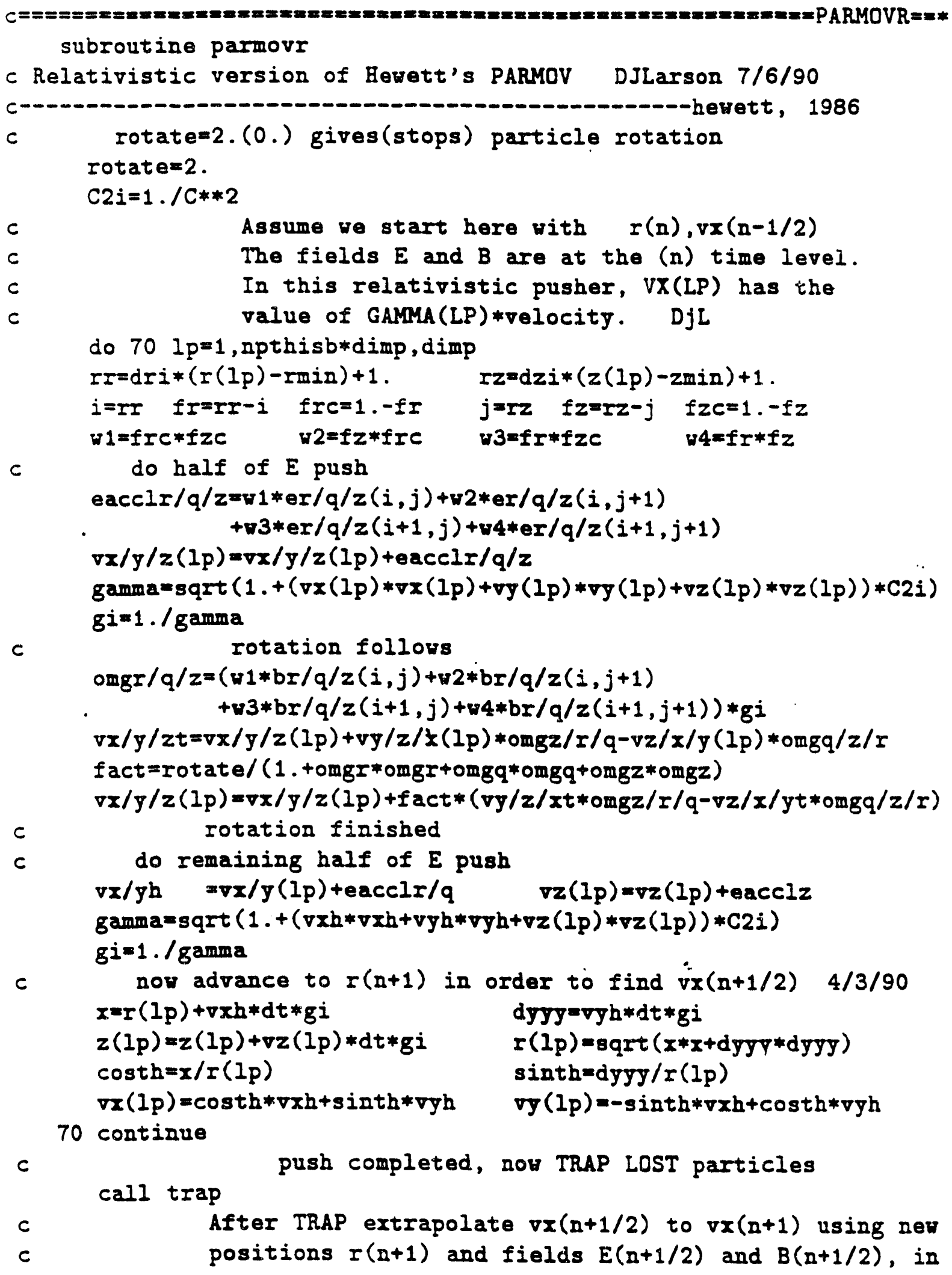


do $2701 p=1$, npthisb*dimp, dimp

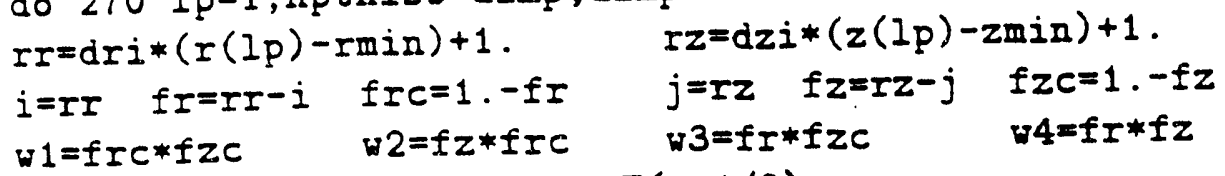

$i=r I \quad f r=r I-i \quad f r c=1 .-f r$

$w 1=f r c * f z c$

*2=fz*fIC

$r z=d z i *(z(I p)-z \min )+1$.

$j=r z \quad f z=r z-j \quad f z c=1 .-f z$

w $3=f I * f z c$

$\forall 4=f I * f z$

order to accumulate sources at the desired time level

do half of $E$ push with $E(n+1 / 2)$

eacclr $/ q / z=.5 *(w 1 * \operatorname{er} / q / z(i, j)+w 2 * \operatorname{er} / q / z(i, j+1)$ $+w 3 * e r / q / z(i+1, j)+w 4 * e r / q / z(i+1, j+1))$

$v x / y / z h=v x / y / z(l p)+e a c c l r / q / z$

gammah $=\operatorname{sqrt}(1 .+(v x h * v x h+\nabla y h * \nabla y h+v z h * v z h) * C 2 i)$

$g i=1 . / g$ ammah

c

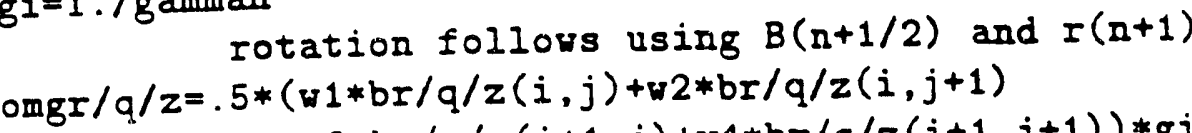
$+w 3 * b r / q / z(i+1, j)+w 4 * b r / q / z(i+1, j+1)) * g i$

$v x / y / z t=v x / y / z h+v y / z / x h * 0 m g z / I / q-v z / x / y h * o m g q / z / r$

fart $=$ rotate $/(1$. +omgr*omgr+omgq*omgq+omgz*omgz)

$v x / y / z h=v x / y / z h+f a c t *(v y / z / x t * 0 m g z / r / q-v z / x / y t * o m g q / z / r)$

do remaining half of $E$ push using $E(n+1 / 2)$

$v x / y /$ in $\quad=v x / y / z h+e a c c l I / q / z$

gammain $=\operatorname{sqrt}(1 .+(v x h * \nabla x h+v y h * \nabla y h+v z h * \nabla z h) * C 2 i)$

$g i=1 . / g$ ammah

$\nabla x / y / z n p 1=\nabla x / y / z h * g i$

$\operatorname{den}(i, j, n)=\operatorname{den}(i, j, n)+w 1$

$\operatorname{den}(i+1, j, n)=\operatorname{den}(i+1, j, n)+w 3$

$\operatorname{den}(i, j+1, n)=\operatorname{cen}(i, j+1, n)+w 2$

$\operatorname{den}(i+1, j+1, n)=\operatorname{den}(i+1, j+1, n)+w 4$

$u r / q / z(i, j, n)=u r / q / z(i, j, n)+w 1 * v x / y / z n p 1$

$u r / q / z(i+1, j, n)=u r / q / z \zeta i+1, j, n)+w 3 * v x / y / z n p 1$

$u r / q / z(i \quad, j+1, n)=u r / q / z(i, j+1, n)+v 2 * v x / y / z n p 1$

$u r / q / z(i+1, j+1, n)=u r / q / z(i+1, j+1, n)+w 4 * v x / y / z n p 1$

tloc $=\nabla x n p 1 * \nabla z n p 1+v y n p 1 * \nabla y n p 1+v z n p 1 * \nabla z n p 1$

$\operatorname{rhs}(i, j)=\operatorname{rins}(i, j)+w 1 * \operatorname{tloc}$

$\operatorname{rhs}(i+1, j)=\operatorname{rhs}(i+1, j)+w 3 * \operatorname{troc}$

$\operatorname{rhs}(i, j+1)=\operatorname{rhs}(i, j+1)+w 2 *$ tloc

$\operatorname{Ihs}(i+1, j+1)=\operatorname{Ihs}(i+1, j+1)+* 4 * t 10 c$

eionr/q/z=eionr/q/z+vx/y/znp $1 * \nabla x / y / z n p 1$

return end 


\title{
Appendix 13. Santa Fe Numerical Simulation Abstract
}

The following was presented at the $13^{\text {th }}$ Conference on the Numerical Simulation of Plasmas in Santa Fe, NM, September, 17-20, 1989.

\section{An Algorithm for Particle Reflection from Internal Boundaries on Orthogonal Uniform Meshes}

\author{
D.W. Hewett, H.L. Rutkowski, ${ }^{*}$ and S. Humphries, Jr. ${ }^{* *}$ \\ University of California \\ Lawrence Livermore National Laboratory \\ Livermore, California 94550
}

Internal structures greatly expand the utility of the PIC method. Recently such structures have been incorporated in an electrostatic R-Z code whose primary mission is to simulate the interaction between a plasma and a wire grid. The grid is held at voltages chosen to confine plasmas density buildups in an optically useful shape until larger external voltages are applied to extract ions. The primary motivation for this effort is the LBL source development effort for proposed heavy ion accelerators that are of interest as possible ICF drivers. We also anticipate using these procedures in codes using more efficient and general algorithms for the Darwin field model (see Larson and Hewett, these proceedings).

The algorithm we describe here is intended to simply detect PIC particles that have moved into regions defined as "inside" a structure. This procedure also serves as a monitor of particles leaving the simulation domain if the external boundary is defined to be a "structure" as well. As a particle incursion is detected, the most difficult task geometrically is to determine which surface it came through and how to reflect it if the entry point is near a corner. Three other commonly particle boundary conditions, absorption, injection/field emission, and thermal reemission, are either less demanding geometrically or special cases of the reflection condition.

In the discussion that follows, it is assumed that we have an orthogonal mesh with uniform spacing and the boundaries of the internal structures as well as the external domain pass through corners of the two dimensional cells.

\section{1) Detection of "lost" particles}

After a group or block of particle has been advanced in time, correct particle physics accounting requires some method to determine if the particle still lies within the acceptable domain. If correct physics is not sufficiently compelling, scattering particle attributes throughout computer memory during the accumulation stage provides stronger motivation. Our procedure is first to determine the cell CENTER indices nearest the particle for each particle and check a structure properties array with those indices, here called $\mathrm{KEY}$ (Icen,Jcen), is to determine the contents of that cell. We use the code 1,0,-1 for plasma, vacuum, and structure, respectively, and the -1 values are set at the start of the run. If KEY(Icen,Jcen)=-1 then the cell center indices closest to the particle's present position are the indices of a cell whose volume is within a structure; therefore the particle

* Lawrence Berkeley Laboratory, Berkeley, CA 94720

** University of New Mexico, Albuquerque, NM 87131 
itself is within that structure and must be processed. If these indices are themselves completely outside the domain of interest. then the particle is truly lost and special hancling is required. Such cases cannot occur if rows and columns of "structures" are defined around the external boundary and if particles are moving less than a cell per time step. Special circumstances can cause the occasional particle far out on the thermal tail to escape-we $\log$ these particles and delete them from the simulation-but if more than a few particles get lost. a through understanding of their origin is needed.

Simple, unoptimized FORTRAN that accomplishes the detection of lost particles for a group of KLAST particles is given here. $\mathrm{k}=0$

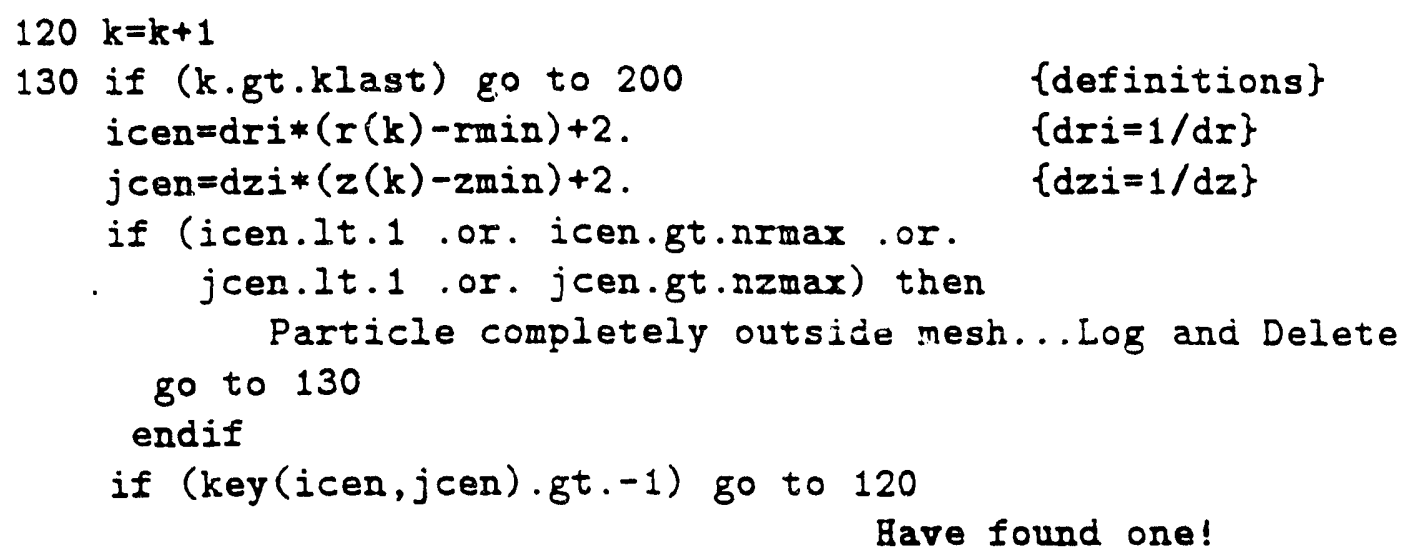

Most particles will generally be inside so that steps requiring the most care in codes for speed (vectorization, parallelizing, etc.) are limited to these steps already discussed. What we do to a particle if it is inside a structure is not as constrained by minimizing CPU cycles. We now discuss handling of those particles that are inside of a structure.

\section{2) Lost particle processing}

The first issue to address after finding a particle in a structure is determine through which surface it passed to get inside. The trick we use. after first verifying that the particle has not moved more than a cell in the last time step, is to move the particle backward in time one-half of a time step and then determine to which cell CORNER it is closest.

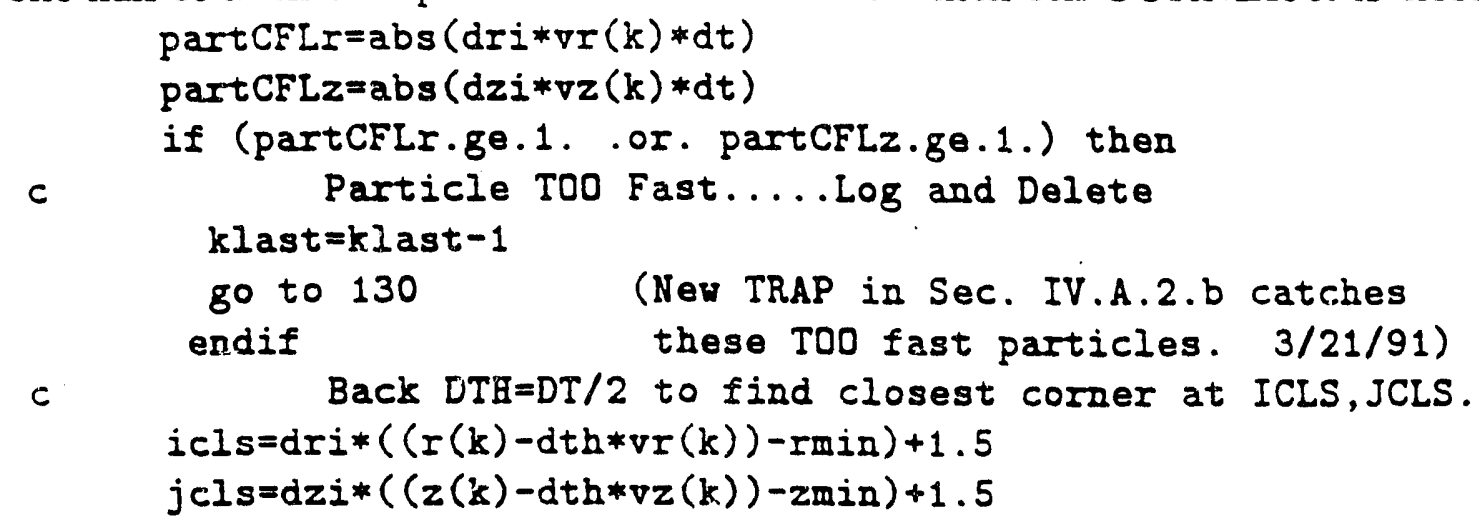

The orientation of the surface through which the particle has passed can be obtained as a function of the closest-corner indices Icls,Jcls from an array KORNT set up at the start of the run. KORNT specifies the surface orientation, as defined in Fig. 1, through the following system: 


$$
\begin{array}{r}
\text { KORNT } \\
+1, \quad \begin{array}{r}
\text { Normal Direction into the PIC Region } \\
+1,+10,-10
\end{array} \\
+11,-11,+9,-9 \quad(+R,+Z), \begin{array}{r}
(-R,-Z),(-R,+Z),(+R,-Z)
\end{array}
\end{array}
$$

The reflections are themseives simple processes. To illustrate, consider $R$-reflections. To reflect the Kth particle in $R$, we reverse the sign of the $R$ velocity and place the particle to the left (or right) of the boundary the same distance it has moved to the right (left) of the boundary. The two FORTRAN statements,

$$
\begin{aligned}
& r(k)=2 . * \operatorname{rgc}(i c l s)-r(k) \\
& \operatorname{vr}(k)=-\operatorname{vr}(k)
\end{aligned}
$$

accomplish the reflection where the R-boundary is located by the grid array RGC(Icls). Remembering that the particle in question is presently inside a boundary cell, we can determine easily when an R-reflection is NOT needed as follows:

a) If $\mid$ KORNT(Icls,Jcls) |=10, the particle has crossed a surface with a surface normal purely in the Z-direction.

b) Only if $[R(k)-R G C(I c l s)] * \operatorname{Vr}(k)>0$ has the particle crossed the R-boundary in question. (This condition is necessary but not sufficient for some cases. see below.)

3) Reflections from Inside and Outside Corners

If the closest cell corner Icls.Jcls has a KORNT that is $+11,+9,-9$, or -11 , either an R- or Z-refiection or both-depending on the particle incident direction-may be required to obtain the particle reflection. If we find a situation in which the criteria for both $\mathrm{R}$ - and Z-reflection are met, both reflections are done. This amounts to reversing the sign of both

\begin{tabular}{|c|c|}
\hline h-reflect if & vr $r<<|v r><0| v r><0|v r\rangle<0$ \\
\hline $\begin{array}{l}(R(k)-R G C(I C L S)) * V R>=0 \\
\text { and }\end{array}$ & $v z><0$ | vz<0 \& $v z<0 \mid v z><0$ \\
\hline $\begin{array}{l}\text { KORNT*(|KORNT } \mid-10) * V R<0 \\
\text { Or } \\
\text { KORNT }=+-1\end{array}$ & $\begin{array}{cl}\text { vr>0 } & |9 / / /| 10 / / / 11 \mid \text { vr } \\
\text { vz><0 } & \mid / / / / / / / / / / / / / \text { vz } \\
& |/ / / / / / /| / / / / / \mid\end{array}$ \\
\hline $\begin{array}{l}Z \text {-reflect if } \\
(Z(k)-Z G C(J C L S)) * V Z>=0 \\
\text { and }\end{array}$ & 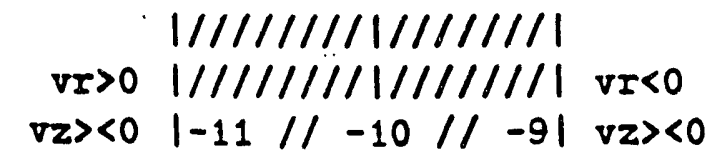 \\
\hline $\begin{array}{l}\text { KORNT } *(! K O R N T !-1) * V Z<0 \\
\text { OI } \\
\text { KORNT }=+-10\end{array}$ & 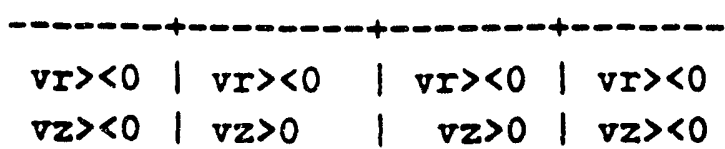 \\
\hline
\end{tabular}
components of velocity and, except for a small lateral translation, the particle is returned in precisely the same direction. We prefer the particle be reflected such that the angle of incidence equal the angle of reflection with respect to the surface normal that, at a corner, depends on the relative size of the grid spacing DR and DZ. The procedure we use, if both and R-and Z-reflection have been done, is to form the dot product of the outgoing velocity $V_{0}$ with the unit vector lying in the

Figure 1

$\mathrm{V}_{0}$ with the unit vector lying in the surface face with components proportional to DR and $\mathrm{DZ}$, respectively. The result is the magnitude of the $V_{o}$ component tangential to the 
face. The desired outgoing velocity is obtained by subtracting this vector twice from $V_{o}$. This algorithm is expressed by the following FORTRAN.

$$
c
$$

ok PERFECT REFLECTION-orient the surface with KORNT

$$
\text { flect } 2=0 \text {. }
$$$$
\text { ikornt=iabs (kornt (icls, jcls)) }
$$$$
\text { if (ikornt.eq. } 1 \text {.or. }((r(k)-r g c(i c l s)) * v r(k)) \cdot g e .0 \text {. and. }
$$$$
\text { kornt (icls,jcls)*(ikornt-10)*vr(k).1t.0)) then }
$$$$
r(k)=2 \text {.*Ige }(i c l s)-r(k)
$$$$
\operatorname{vr}(k)=-v r(k)
$$$$
\text { flect } 2=2 . /(d r * d r+d z * d z)
$$

endif

if (ikornt. eq.10 .or. $((z(k)-z g c(j c l s)) * \nabla z(k)) \cdot g e .0$. . and.

kornt (icls, jcls)*(ikornt- 1)*vz(k).lt.0)) then

$z(k)=2 * z g c(j c l s)-z(k)$

$v z(k)=-v z(k)$

$c$ Have we already done an R-reflection? If so fix outgoing.

$$
\begin{aligned}
& s d z=(10-i k \circ r n t) * d z \\
& s v r=\operatorname{vr}(k)-f l e c t 2 * d r *(\operatorname{vr}(k) * d r+v z(k) * s d z)
\end{aligned}
$$$$
v z(k)=v z(k)-f l e c t 2 * s d z *(v r(k) * d r+v z(k) * s d z)
$$$$
\operatorname{vr}(k)=s v r
$$

endif

c

Next Particle, Please..

go to 120

200 continue

The extra logic involving KORNT is less straightforward but is needed to manage special cases that arise when inside corners as well as outside corners ( 3 quadrants of "structure" for inside, only 1 for outside) are considered. The differences arise from those particles that lodge in one of the two extra quadrants, thus needing one but not both reflections.

The next task is to incorporate these ideas into a vectorized particle pusher.

\section{Acknowledgements}

Work performed under the auspices of the United States Department of Energy by the Lawrence Livermore National Laboratory under contract number W-7405-ENG-48. 
Appendix 14. GYM28 Summary Sheets

$$
\text { GYMNOS (GYM28) HELP 1/3/91 units----CGS, eV }
$$

parameter ( $n r 2=i 3, n z 2=19, \operatorname{mxg} 1 \mathrm{~d}=73, \mathrm{nstmax}=8$, ithmx $=2000, \mathrm{kcormx}=700$ )

parameter (nheadb $=10$, mpbuf $=1000$, dimp $=5$, memol $y=80160, n s p=2$ )

For disk storage, just set MEMOLY=0. For memory storage,

MEMOLY $Y=\operatorname{sumNSP}[1+(\operatorname{MSPEC}(n) /$ MPBUF $)] *(N B E A D B+$ MPBUF $* D I M P)$.

NREST $!=0$ write a restart every |NREST| DTs and at TIME $=$ TMAX.

$=0$ no restart read or written. <0 start from restart

NUMREST is always the restart number you will write next time.

IMSG $=1[0]$ do[n't] process keyboard messages: choices IOTTY $N$ ABORT

AUTOVLM auto phase space $(V x-x)$ plot scaling

= 1. includes 0 and "growth" factor over max from IT-1

$=-1$. scaling will stretch but not shrink $!=|1$.$| fixed$

///GEOSET----------STRUCTURE SPECIFICATION ARRAYS--

RG ZG VOL(Rmn,Rmx corrected) cell cornrs RGCC ZGCC VOLCC cell cntr

NSTRUCT(nstr,3) STRGEN(nstr,6) KCIJ(kCor,2) KCORR(ijcor)

$\operatorname{KORNT}(i j$ COr) SKEY(ijcen) KEY(ijcor)

User sets NSTRUCT (nstr,1) and STRGEN (can read with JSTGNSW)

calls GEOMSET to set NSTRUCT(nstr,2/3), KCORR, KCIJ->SKEY->KORNT \& KEY NSTRUCT(NSTR,1)--name of structure numbered NSTR

PIE STRGEX(NSTR,1/2/3/4/5/6) $1 /$ rcen/zcen/radius/astr/astp ( $a=0$ e_r)

BAR STRGEN (NSTR, 1/2/3/4/5/6) 2/rstr/zstr/rstp/zstp/hwidth

NSTR=1,2,3,4 generally Rmin,mx, $\mathrm{Zmn}, m x$ CORMMM $/ M X=0$ (1) nclds cornr

CAUTION: Boundaries are built only at cell edges!

JSTGNSW=1 use STRGEN, NSTRUCT, NSTRMX as set in BCSET or $=0(-1)$ read tty(6)

NSTRUCT(NSTR, 2/3)--KCOR start/stop pt in KCIJ.

ex: do \#kcor=nstruct (nstr, 2), nstruct (nstr, 3)

$\# \operatorname{key}(\operatorname{kcij}(\operatorname{kcor}, 1), \operatorname{kcij}(\operatorname{kcor}, 2))=-1$

IorJCOR=KCIJ (KCOR, 10r2) for structure corner pt KCOR(=KCORR(ijCOI) xIef)

-

$\operatorname{KORNT}(i c o r, j c o r)=0$ not a B surface pt.

$=+1(-1)$ for surface normal in for(back) ward R-direction

$=+10(-10)$ for surface normal in for(back) ward $Z$-direction

\begin{tabular}{|c|c|c|c|}
\hline$/ 1+11$ & $-\infty-\infty+$ & $+9 \mathrm{~K} /$ & $+--\infty$ \\
\hline $1+\cdots--$ & $1 / 1 / 1 /$ & $--\cdots+1$ & $1 / / / / 1$ \\
\hline $1 / 1 / 11$ & $+12 / /$ & $1 / 1 / 1 /$ & $1 /+8$ \\
\hline
\end{tabular}

$\begin{array}{llll}\mid /-12 & / / / / / & -8 / 1 & / / / / 1 \\ \mid / / / / & ---+1 & / / / / 1 & /+\cdots \\ +\cdots-- & -11 / / & ---+ & / 1-9\end{array}$

Plasma GEOMETRY ARRAYS--(plasma/vacuum set in DENSET)

$\operatorname{SKEY}(i c e n, j$ cen $)=+1$. if DEN $>=C T O F F$ (in plasma) $=0$. if vacuum

$=-1$. if IN boundry (only 1./0. in periodic ghost cell)

KEY (icor,jcor) $=+1$ if in the plasma, $=0$ if in a vacuum 
$=-1$ if ON or IN bndry (periodic bndry loro only)

///PBCSET--- - - - - PRTCL/BEAM SPECIFICATION ARRAYS

$\operatorname{BEAM}(n s t r, n s p, 8) \quad \operatorname{IPBC}$, QABSRB (ijcor) TEM(ijcor,nsp)

UNBSTR , TEMPSTR, QBRS (kCOI, lord, nsp)

Given NSTRUCT,KCIJ, the user inputs NSTPROP TEMIN BEAM,

\& this routne sets IPBC UNBSTR TEM TEMPSTR QBRS NPFEMT NPNJCT

NSTPROP (NSTR, N species) --n00-n50, n51--\%THREMT, PRFLCT

( $n=1 / 2 / 0$ Femt/Njct/Neither), negative=no dithr

$\operatorname{IPBC}(i j c o r)=$ =ell corner particle property = last 2 digits NSTPROP.

61 returns IFF reduces QABSRB total on surface. (NOT YET ever?)

QABSRB (icor,jcor) = Absorbed charge at this CELL CORNER.

THREMT... Reemit .02*|IPBC $\mid * Q A B S R B / S Q$ using TEM \& ETNVTP

(from BEAM(nstr,n,4) ) \& DITHR from sign of NSTPROP(nstr,n).

Normal velocity $=0$

$\operatorname{TEM}(i j \operatorname{cor}$, lord,$\Omega)=$ TXVIS .

NJCT/FEMT...reservoir charge QBRS(kcor,LorD, $n$ ) at this CELL FACE.

LorD $=1(2)$ [emitting surface Left(Down) from corner]

BEAM(nstr, $n, 1 \& \quad 2 \& \quad 3 \& \quad 4 \& \quad 5 \& \quad 6 \& \quad 7 \& \quad 8$ )

Bden,unbstr, temstr, etnvtp, turnon, rise,turnof,fall

UNBSTR (kcor, lord, $n$ ) $=$ Nrml vlcty mag. TEMPSTR (kcor, lord, $n$ ) $=$ Txvrs .

Tnormal/Txvrs from BEAM(nstr, $n, 4)$ \& DITHR from sgn NSTPROP for all.

E.G., to absorb \& THREMT $50 \%$ of the prtcls species $n=3$ that hit NSTR \&

njet a DITHRed beam with Bden=1e12 \& Vnormal=3C/4 \& $3 \mathrm{eV}$ xverse thermal, on instantly after $1.5 \mathrm{~ns}$, remaining on until 5 ns \& falling linearly to 0 by 6.2 ns with anisotropy Tn/Txrs of $30 \%$, use $\operatorname{temin}(3)=3$. nstprop (nstr, 3) $=225$ beam (nstr, 3,1)=1.e12 beam(nstr, 3, 2) =7.5e9 beam $(n s t r, 3,3)=3$. beam (nstr, 3,4)=.3 beam (nstr, 3,5)=1.5e-9 beam $(n s t r, 3,6)=0$. beam (nstr, 3,7) $=5 \cdot e-9 \quad$ beam (nstr, 3, 8) $=1.2 e-9$

///FLDSET-----Given NSTRUCT, KCIJ user sets BV,BVR,BVZ \& KN (aII IJCOR)

$\mathrm{KN}(\mathrm{icor}, j \operatorname{cor})=0$, Dirichlet pt with value=BV(icor,jcor) on\&in bndry. $=+1(-1)$ on bndry w/ for(back) ward R-drivt $[=+2(-2)$ curl-tpoel $=K N+10(-10)$ on bndry $w /$ for(back) ward $Z$-drivt $B V R / Z$ (icor,jcor) = Neumann value for the $R / Z$ derivative. VOLT(NSTR, R/Q/Z,1/2/3/4/5) mag/turnon/rise/turnof/fall PHISTR(NSTR, 0/1/2/3/4/5) mago/mag/turnon/rise/turnoff/foff MAG is the voltage ultimately added to MAGO ///INIT/INPROF---INPROF quantites for prtel ntlzatn ars CELL CENTERED! $\operatorname{NPINIT}(n)=N P \ldots$ of sprtels rqustd......NSPEC $(n) \ldots \#$ at any time $\operatorname{RPPSP}(n)$ is obtained from $\operatorname{TOTNRP/NSPEC}(n)$ at time=0 if $\operatorname{NPINIT}(n) !=0$ If $\operatorname{NSPEC}(n)=0$, assume all injected beam densities uniformly fill region to give REFNRP then $\operatorname{RPPSP}(n)=\operatorname{REFNRP/MSPEC}(n)$.

def: Vth=sqrt(boltzk TEM/smsngl( $n)$ ) Vmp=sqrt ( 2 boltzk TEM/smsngl( $(n)$ ) Pressure=VD3 DEN boltzk TEM VD3 is the velcty space dimensnality ipbuf (1)=numbuf $\quad$ ipbuf $(2)=n p t h i s b \quad i p b u f(3)=n b s p e c(n) \quad$ ipbuf $(4)=n$ 
ipbuf (5)=namsp(n) ipbuf(6)=it $\quad \operatorname{pbufh}(8)=s q(n) \quad \operatorname{pbufh}(9)=s m(n)$

///TRANS-----orchastrates particle advance and source accumulation

TRANS converts "raw counts" to densities by dividing by EFFVOLs with

by Rfactors at $R$ bndys complete with in/outside corner distinctions.

RFACOT $=3(4 \mathrm{rg}(i)+d r) / 4(3 \mathrm{rg}(i)+d r) \quad \operatorname{RFACIN}=3(4 \mathrm{rg}(i)-d r) / 4(3 \mathrm{rg}(i)-d r)$

Tro species plotted as same species if a) they are adjacent in the buffering, b) they have the same $\operatorname{NAMSP}(n)$, \& $c$ ) they have the same $S Q(n)$ \&M(n)

///PARMOVers-----AIl movers use Boris that assumes we start with $R(n), V X(n-1 / 2)$ \&ields $E$ and $B$ are at the (n) time level.

$\begin{array}{lll}\text { PARMOV VPARMOV } & \text { PARMOVE } & \text { PARMOVR } \\ \text { fuIIEM fuIIEMV } & \text { ES } & \text { fullEMrelativistic }\end{array}$

$E$ \& $B$ have been initialized by TRANS to carry QVM*DTH*E \& QVM*DTH*B/C

Calls TRAP after prtcls pushed to 1) insure none have invalid

locations, 2) to reflect or absorb/THREMT or FEMT or NJCT

Tried to rotate the $n+.5$ velctis back to $Q=0$ with $n+.5$ positions but-changed to original Boris algorithm using $n+1$ positions to avoid a density pile-up on axis. $4 / 3 / 90$

///TRAP---insures valid prtcl locations/ IPBC(ijcor)--set for each NSP If a prtcl is in cell where SKEY is -1 , , move or deleted it.

If perfect reflection, put back inside with bounce off boundary, even if prtcl exceeds CFL. Deleted partcls add charge to QABSRB(ijcor)

Perfect reflection for prtcls with CFL>1 via back cycles with smaller CFLok DTCLS to find where last prtcl crosing occured. Multiple passes catch those whose rflctn puts them in yet another structure.

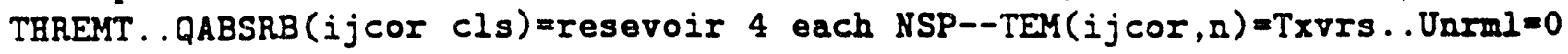
Reemit $.02 * \mid$ IPBC $\mid * Q A B S R B / S Q \ldots \ldots \ldots \ldots \ldots \ldots \ldots \ldots \ldots \ldots \ldots \ldots \ldots$ CORMERS N JCT/FEMT . . QBRS, TEMPSTR, UNBSTR (kcor, lord, $n$ ) . . resevoir, Txvrs, UnIml . FACES Tnormal/Txvrs from BEAM(nstr,n,4) \& DITHR from sgn NSTPROP for all. c JK Boyd's trick to FEMT .25 cell away from bndy (elso den infinite) 

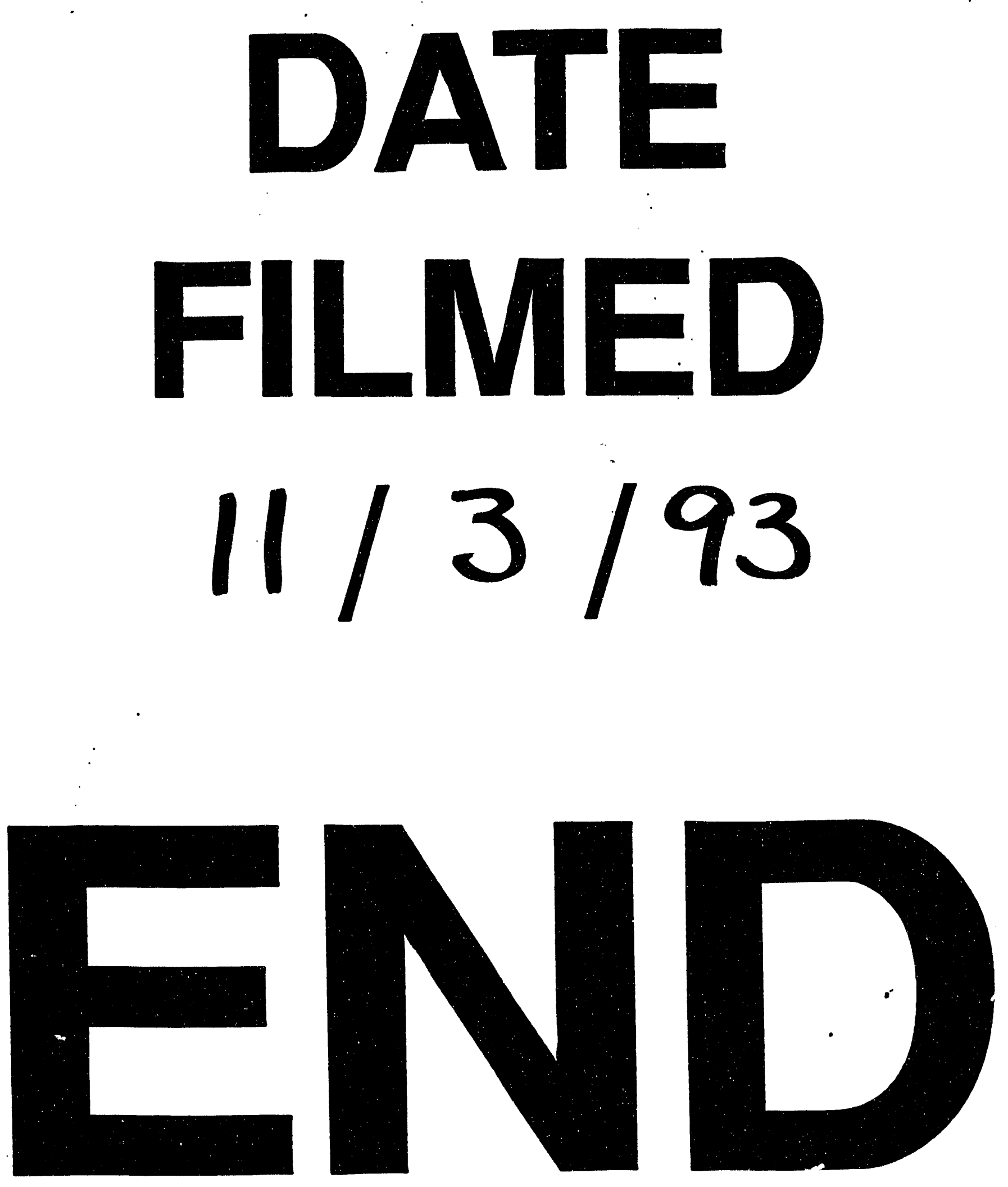
